\title{
Kynurenine analog 3-HAA is a ligand for transcription factor YY1
}

\section{Zhaopeng Shi}

Shanghai Jiao Tong University School of Medicine

\section{Guifang Gan}

Shanghai Jiao Tong University School of Medicine

\section{Xiang Xu}

Shanghai Jiao Tong University School of Medicine

\section{Jieying Zhang}

Shanghai Jiao Tong University School of Medicine

\section{Yuan Yuan}

Shanghai Jiao Tong University School of Medicine

\section{Bo Bi}

Shanghai Jiao Tong University School of Medicine

\section{Xianfu Gao}

Shanghai Profleader Biotech Co., Ltd

\section{Pengfei Xu}

Xiangya School of Pharmaceutical Sciences, Central South University

\section{Wenbin Zeng}

Xiangya School of Pharmaceutical Sciences, Central South University

\section{Jixi Li}

Fudan University https://orcid.org/0000-0003-3463-3175

\section{Youqiong Ye}

Shanghai Jiao Tong University School of Medicine https://orcid.org/0000-0001-8332-4710

\section{Aiwu Zhou}

Shanghai Jiao Tong University School of Medicine https://orcid.org/0000-0002-2555-5091

\section{Naixia Zhang}

Shanghai Institute of Materia Medica, Chinese Academy of Sciences, Shanghai, China

\section{Wen Liu}

Xiamen University https://orcid.org/0000-0003-3434-4162

\section{Shu-hai Lin}

Xiamen University

Jun Mi ( $\nabla$ jmei@sjtu.edu.cn )

Shanghai Jiao Tong University https://orcid.org/0000-0001-5788-3965 


\section{Article}

Keywords: 3-hydroxyanthronic acid (3-HAA), kynurenine, YY1, DUSP6, Hepatocellular Carcinoma (HCC), tryptophan metabolism

Posted Date: October 21st, 2020

DOI: https://doi.org/10.21203/rs.3.rs-92311/v1

License: (c) (i) This work is licensed under a Creative Commons Attribution 4.0 International License.

Read Full License 


\section{Kynurenine analog 3-HAA is a ligand for transcription factor YY1}

Zhaopeng Shi ${ }^{1, \#}$, Guifang Gan ${ }^{1, \#}$, Xiang Xu ${ }^{1}$, Jieying Zhang ${ }^{1}$, Yuan Yuan ${ }^{1}$, Bo Bi ${ }^{2}$, Xianfu Gao ${ }^{3}$, Pengfei $\mathrm{Xu}^{4}$, Wenbin Zeng ${ }^{4}$, Jixi $\mathrm{Li}^{5}$, Youqiong Ye ${ }^{1}$, Aiwu Zhou ${ }^{1}$, Naixia Zhang ${ }^{6}$, Wen Liu ${ }^{7, *}$, Shuhai $\operatorname{Lin}^{7, *}$, Jun $\mathrm{Mi}^{1,2, *}$

${ }^{1}$ Basic Medical Institute; Key Laboratory of Cell Differentiation and Apoptosis of the Chinese Ministry of Education; Hongqiao International Institute of Medicine, Tongren Hospital, Shanghai Jiao Tong University School of Medicine

${ }^{2}$ Department of Nuclear Medicine, Rui Jin Hospital, Shanghai Jiao Tong University School of Medicine

${ }^{3}$ Shanghai Profleader Biotech Co., Ltd

${ }^{4}$ Xiangya School of Pharmaceutical Sciences, Central South University

${ }^{5}$ Fudan University School of Life Science

${ }^{6}$ CAS Key Laboratory of Receptor Research, Department of Analytical Chemistry, Shanghai Institute of Materia Medica, Chinese Academy of Sciences, Shanghai, China

${ }^{7}$ School of Pharmaceutical Sciences, State Key Laboratory of Cellular Stress Biology, Xiamen University

\# These authors contributed equally to this work

* Correspondence to: Jun Mi, jmei@sjtu.edu.cn; Shuhai Lin, shuhai@xmu.edu.cn; Wen Liu, w2liu@xmu.edu.cn

Key words: 3-hydroxyanthronic acid (3-HAA); kynurenine; YY1; DUSP6; Hepatocellular Carcinoma (HCC); tryptophan metabolism 


\section{ABTRACT}

Kynurenine, a metabolite of tryptophan, promotes immune tolerance in development and tumor evasion by binding to the aryl hydrocarbon receptor (AHR). However, the IDO inhibitors, blocking kynurenine generation, fail in stage III of clinical trials in several tumors for unknown reasons. Here, we report that 3hydroxyanthranilic acid (3-HAA) induces apoptosis and synergizes with IDO inhibitors by increasing the suppression of IDO inhibitors on HCC xenograft growth. The content of 3-HAA, a catabolite of kynurenine, is lower in tumor cells by downregulating its synthetic enzyme $\mathrm{KMO} / \mathrm{KYNU}$ and/or upregulating its catalytic enzyme HAAO. Overexpression of KMO suppresses tumor formation and tumor growth by increasing endogenous 3-HAA while adding exogenous 3-HAA also inhibits tumor growth. Moreover, we found that 3-HAA directly binds transcription factor YY1 rather than AHR and increasing the PKC $\zeta$ phosphorylation of YY1 at the Thr 398 in response to 3-HAA; YY1 phosphorylation at T398 increases the YY1 binding to chromatin. 3-HAA-induced Thr398 phosphorylation of YY1 upregulates the expression of dual-specificity phosphatase 6 (DUSP6), etc. DUSP6 overexpression induces apoptosis of hepatocellular carcinoma (HCC) cells and suppresses the HCC growth in vitro and in vivo. The T398 phosphorylation of YY1 is critical for the 3-HAA-induced apoptosis in tumors. These findings demonstrate that kynurenine analog 3-HAA is a functional metabolite associating YY1 as an endogenous ligand, downregulation of 3-HAA is necessary for the rapid growth of tumor cells, suggesting its promising approach in HCC therapy.

Highlights:

1. 3-HAA selectively downregulated in tumor cells;

2. 3-HAA induced apoptosis of HCC cells by upregulating DUSP6;

3. 3-HAA directly binds transcription factor YY1;

4. PKC $\zeta$ phosphorylates T398 of YY1 in response to 3-HAA. 
The essential amino acid tryptophan is catabolized mainly through the serotonin pathway in the brain or the kynurenine pathway in the liver (Chen and Guillemin, 2009; Schrocksnadel et al., 2006).

Tryptophan metabolism is enhanced in various tumors by upregulating the expression of indoleamine 2,3dioxygenase 1/2 (IDO1/2), the rate-limiting enzyme in the kynurenine pathway. Kynurenine, a catabolite of tryptophan, increases immune tolerance in development and disease by directly binding to the aryl hydrocarbon receptor (AHR) (Opitz et al., 2011; Sharma et al., 2007), and enhances tumor immune evasion to promote tumor growth (Opitz et al., 2011) (Li et al., 2011) (Schwarcz et al.). The 3hydroxyanthranilic acid (3-HAA), a derivative of kynurenine was reported to exert anti-inflammatory effects by selectively inducing the apoptosis of activated T cells (Krause et al., 2011; Lee et al., 2010). However, the function of other kynurenine derivatives largely remains unclear. Here, we report 3-HAA, selectively decreased in various tumors, regulates the activity of transcription factor YY1 by directly binding and consequently induces tumor cell apoptosis and suppresses HCC growth in vitro and in vivo.

\section{3-HAA is decreased in tumor cells}

To comprehensively understand the effect of kynurenine derivatives on tumor, tryptophan catabolites were first analyzed in clinical HCCs using liquid chromatography-tandem mass spectrometry (LCMS/MS) and gas chromatography-mass spectrometry (GC-MS). The concentration of kynurenine catabolite 3-HAA decreased in 37 cases of HCC ( $p<0.01)$ and 42 cases of esophageal carcinomas $(\mathrm{p}<0.01)$ compared to the matched paratumor tissues (Figs. 1A and S1A). Conversely, the concentration of tryptophan and kynurenine was higher in these HCCs and esophageal carcinomas than in the matched paratumor tissues, respectively. There was no significant difference in 3-hydroxykynurenine (HK) between tumors and adjacent non-cancerous tissues. Consistent with this observation, the concentration of 3-HAA was also lower in seven HCC cell lines tested than in normal hepatic LO2 cells, whereas the content of tryptophan and kynurenine increased in these tested HCC cell lines (Fig. 1B). Immunohistochemical analysis further confirmed lower 3-HAA content in clinical HCC tissues than in adjacent non-cancerous tissues (Fig. 1C).

Metabolic flux analysis using labeled tryptophan revealed catabolism into kynurenine but not HK or 
3-HAA in HCC cells, and the newly generated kynurenine was secreted into the culture medium (Fig. 1D). Although the expression of 3-HAA degrading enzyme hydroxyanthranilate 3,4-dioxygenase (HAAO) varied in HCC cells, both western blotting and immuno-histochemical analysis showed that kynurenine 3-monooxygenase (KMO) and kynureninase (KYNU), enzymes converting kynurenine into 3-HAA, were downregulated in HCC cells (Fig. 1E \&S1B). The analysis of HCC expression profile in the TCGA database also supported our finding (Fig. S1C), suggesting tumor cells reprogram the tryptophan metabolism by modulating the upstream/downstream catalytic enzymes.

Furthermore, overexpression of KMO or knockdown of HAAO significantly increased the concentration of 3-HAA in HCC SMMC7721 cells, 6 times more than control cells, but not the HK, picolinate (PA), or quinolinate (QA) (Fig. 1F \& S1D). These observations suggest that 3-HAA is selectively downregulated in cancer cells by downregulating KMO expression and/or upregulating HAAO.

\section{3-HAA inhibits tumor growth by inducing apoptosis}

To determine whether 3-HAA could inhibit tumor growth, exogenous 3-HAA was added to HCC cultures, and cell proliferation was assessed. Indeed, 3-HAA significantly inhibited HCC cell growth and colony formation (Figs. 2A and S2A). In contrast, the other tryptophan metabolites Kyn, PA, and QA did not substantially affect growth or colony formation of HepG2 or SMMC7721 cells. To verify that this effect was caused by 3-HAA and not its substrate, the cellular concentrations of 3-HAA and kynurenine were analyzed by LC-MS/MS in 3-HAA-treated tumor cells. Although the content of endogenous 3-HAA varied among different HCC cell lines, 3-HAA treatment increased the cellular concentration of 3-HAA, particularly in SMMC7721 and HepG2, and the downstream substrate but not kynurenine (Fig. S2B).

To understand the mechanism by which 3-HAA inhibited HCC cell growth, gene expression profiling of 3-HAA-treated HCC cells was analyzed by the gene ontology (GO) enrichment. The GO analysis revealed that the cell death-related pathways were highly activated in 3-HAA-treated HCC cells (Fig. 2B). To further determine what signal mediates 3-HAA-induced cell death, tumor cells were treated with 3-HAA and then analyzed for markers of apoptosis, autophagy, and necrosis (Fig. S2C). Apoptosis 
was increased in a dose-dependent manner by TUNEL assay in the SMMC7721 cells (Fig. 2C). Levels of cleaved caspase 3 and cleaved PARP were increased in a dose- and time-dependent manner in the SMMC7721 and HepG2 cells (Fig. 2D). However, 3-HAA treatment did not significantly alter levels of autophagy markers LC3-II and p62 or necrosis marker RIP3 in the SMMC7721 or HepG2 cells (Fig. $\mathrm{S} 2 \mathrm{C})$.

Furthermore, the apoptosis inhibitor ZVAD, but not the necrosis inhibitor Nec1 or autophagy inhibitor 3-MA, restored growth of HepG2 and SMMC7721 cells following 3-HAA treatment (Fig. 2E). This effect was observed at ZVAD doses of 50 and $100 \mu \mathrm{M}$. 3-HAA treatment also increased apoptosis in HCC xenografts, as observed based on TUNEL foci and flow cytometry of Annexin V (Figs. 2F and 2G). Consistent with these results, 3-HAA slowed tumor growth (Fig. S2D).

To determine the effect of endogenous 3-HAA on HCC tumor formation, the cell growth was analyzed in SMMC7721 cells overexpressing KMO or knocking down HAAO enzymes. Either overexpression of KMO or knock down of HAAO inhibited cell growth of HCC cells (Fig. 2H \& S2E). Moreover, the tumor formation assay showed KMO overexpression suppressed tumor formation and tumor growth in HCC xenograft model, (9/10) versus (4/10) (Fig. 2I, S2F \& S2G). The flow cytometry analysis showed that overexpression of KMO increased apoptosis of HCC cells, two times more than control cells (Fig. 2J \& S2H). Remarkably, the Kaplan-Meier survival analysis showed that HCC patients with high KMO expression had longer disease-free survival than patients with low KMO expression (Fig. $2 \mathrm{~K})$. These observations suggest that 3-HAA is a negative regulator for tumor formation and tumor growth.

\section{3-HAA suppressed tumor growth by upregulating DUSP6 expression}

To determine the mechanism by which 3-HAA induces tumor cell apoptosis, RNA sequencing was used to profile gene expression in SMMC7721 or HepG2 cells after 0, 1, 8, or 24 h treatment with 3-HAA according to the screening strategy previously applied (Opitz et al., 2011). At all three time points after the start of 3-HAA treatment, the top 21 upregulated genes were selected (Fig. 3A). The expression of the top seven of these genes was individually verified by real-time PCR. Immunoblotting of the top two 
upregulated genes also showed elevated levels of DUSP6 and IGFBP1 in SMMC7721 and HepG2 cells (Fig. 3B). These results suggest that 3-HAA alters the gene expression profile of HCC cells, with DUSP6 and $I G F B P 1$ as two of the most upregulated genes. However, the clinical data showed that the overall survival of HCC patients was only associated with the expression level of DUSP6 $(\mathrm{p}<0.05)$, but not IGFBP1 ( $\mathrm{p}>0.05)$. Patients expressing a high level of DUSP6 were longer than patients expressing a low level of DUSP6 $(\mathrm{p}<0.05)$ (Fig. 3C and S3A). The multivariate analysis showed that DUSP6 was a major factor in the HCC patients' survival among the top 4 upregulated genes (Fig. S3B), and the corrective analysis with the clinical characteristics also supported this finding (Fig. S3C), indicating that DUSP6 is important to 3-HAA-induced apoptosis.

To demonstrate whether DUSP6 mediates 3-HAA-induced tumor cell apoptosis, the effects of DUSP6 on HCC cell growth were first analyzed in HCC cells. DUSP6 knockdown restored growth of HepG2 and SMMC7721 cells inhibited by 3-HAA (Figs. 3D and S3D), whereas it did not affect the growth of untreated HCC cells (Fig. S3E). 3-HAA induced apoptosis to a smaller extent in DUSP6depleted SMMC7721 cells than in the control cells, based on flow cytometry using Annexin V (Fig. 3E). DUSP6 depletion was also associated with reduced levels of cleaved caspase-3 and cleaved PARP, as well as enhanced ERK activity (Fig. 3F). In fact, DUSP6 knockdown restored ERK activity that had been suppressed by 3-HAA (Fig. 3F). ERK enhancement inhibits apoptosis via BAD/BCL2/BCL-XL signaling (Piya et al., 2012). More impressively, DUSP6 knockdown reversed 3-HAA-mediated suppression of tumor growth in SMMC7721 xenografts (Fig. 3G).

\section{3-HAA upregulated DUSP6 expression by binding with transcription factor YY1}

To determine which transcription factor or co-activator mediates 3-HAA regulation of gene expression, the 38 common transcription factors or co-activators were first selected from those proteins potentially bind to the promoter region $(-5000$ to +1$)$ of top 4 genes (http://gtrd.biouml.org) (Yevshin et al., 2019) (Fig. 4A and S4A). Moreover, levels of chromatin proteins in 3-HAA-treated SMMC7721 and HepG2 cells were quantified by tandem mass-tagged quantitative proteomics analysis (TMT). The 91 proteins consistently and increasingly bound to chromatin at the $1^{\text {st }}$ hour and the $8^{\text {th }}$ hour post 3 -HAA 
treatment (Fig. S4B), and YY1 was the only protein overlapped with the predicted transcription factors that potentially bind to the promoter region of the top 4 genes (Figs. 4B). Both the immunoblotting of nuclear fraction and immunofluorescent staining also showed that 3-HAA increased YY1 nuclear accumulation (Fig. 4C \& S4C). Moreover, analysis on gene expression profile showed that about $16 \%$ of 3-HAA-regulating genes were the same with YY1-targeting genes, including DUSP6 (Fig. S4D), suggesting transcription factor YY1 could regulate DUSP6 expression after 3-HAA treatment.

Indeed, YY1 knockdown abolished 3-HAA-induced upregulation of DUSP6 in SMMC7721 cells (Fig. 4D). After 3-HAA treatment, the level of cleaved Caspase 3 and cleaved PARP was decreased in SMMC7721 cells depleted of YY1, and recovered by DUSP6 overexpression (Fig. 4E). The TUNEL assay and flow cytometry analysis demonstrated that 3-HAA-induced apoptosis was also reduced in SMMC7721 cells depleted of YY1, overexpression of DUSP6 restored the apoptosis suppressed by YY1 depletion (Fig. S4E, S4F \& 4F). Eventually, YY1 knockdown restored SMMC7721 cell growth suppressed by 3-HAA, and overexpressing DUSP6 inhibited YY1-depleted cell growth again (Fig. 4G), suggesting YY1 mediates the 3-HAA upregulation of DUSP6. Also, adding 3-HAA did not affect the expression of CYP1A1, a target gene of AHR (aryl hydrocarbon receptor) (Fig. S4G), suggesting that 3HAA functionally overlaps with YY1, but not the AHR, which was distinct from kynurenine.

Since YY1 is a transcription factor, we speculated that 3-HAA could regulate YY1 transcription activity on DUSP6 gene promoter. Closer analysis of the DUSP6 promoter region using online-based prediction tools (jaspar.genereg.net and ecrbrowser.dcode.org) (Khan et al., 2018a; Khan et al., 2018b) revealed a novel potential YY1 binding DNA fragment at positions -1,145 to -1,134 (TCCATCCGGCTT), which is distinct from the reported consensuses binding sequence (CAANATGGCGGC) (Kim and Kim, 2009). To determine whether YY1 regulates DUSP6 expression by binding this novel sequence, each DNA fragment was added to a luciferase reporter gene, and YY1driven luciferase expression was measured by its enzyme activity. Higher luciferase activity was observed with the full length or partial DUSP6 promoter containing this novel specific sequence as well as the consensus YY1 binding sequence. Luciferase activity decreased when mutations involving this novel 
binding site (mut2) occurred (Fig. 4H). 3-HAA increased YY1 binding to this novel binding sequence in a dose-dependent manner, as evidenced by an in vitro electrophoretic mobility shift assay (Fig. 4I).

Moreover, the quantitative PCR analysis following chromatin immunoprecipitation of YY1 revealed that 3-HAA promoted YY1 binding to the consensus sequence of $p 53$ promoter region (positive control) and the novel binding sequence in the DUSP6 promoter region, as reflected by 3-HAA-induced YY1 enrichment (Figs. 4J and S4H). ChIP sequencing analysis further confirmed that 3-HAA induced the union peak formation of YY1 on the promoter region of DUSP6, IGFBP1, NROB2 and IER3 genes (Fig. $4 \mathrm{~K} \& \mathrm{~S} 4 \mathrm{I})$.

To determine whether 3-HAA promotes DUSP6 expression by binding YY1, we first tested whether 3-HAA directly associates with YY1 in vitro using nuclear magnetic resonance. Dose-dependent signal attenuation was observed in the T1r NMR spectrum, and positive saturation transfer difference (STD) signals were detected in the STD spectrum, suggesting that YY1 interacts with 3-HAA (Fig. 4L). Surface plasmon resonance experiments suggested a moderate binding affinity with $K D$ of $121.7 \mu \mathrm{M}$ (Fig. 4M). This binding was further confirmed by microscale thermophoresis (MST) analysis (Fig. S4J).

\section{PKC $\zeta$ phosphorylates YY1 at Thr 398 in response to 3-HAA}

Previous studies showed that phosphorylation regulated YY1 DNA binding activity (Daub et al., 2008; Kaludov et al., 1996). Thus, the phosphorylation of YY1 were first analyzed 2 hours after 3-HAA treatment to determine whether 3-HAA induced YY1 phosphorylation. The immunoblotting following phosphorylated protein enrichment showed that 3-HAA treatment also increased YY1 phosphorylation, and mass spectrometry analysis revealed that the T398 of YY1 were phosphorylated by 3-HAA (Fig. 5A). The T398A mutation diminished the 3-HAA-induced YY1 nuclear accumulation and YY1 T398 phosphorylation (Fig. 5B \& 5C). The T398 phosphorylation specific antibody was developed for this detection.

The function analysis displayed that the T398A mutation of YY1 suppressed 3-HAA-upregulated DUSP6 expression and reduced the level of cleaved Caspase 3/cleaved PARP, whereas the mimic phosphorylation of T398E mutation promoted DUSP6 expression even without 3-HAA treatment (Fig. 
5D). The TUNEL assay and the flow cytometry analysis demonstrated that the T398A mutation of YY1 suppressed 3-HAA-induced apoptosis (Fig. 5E \& S5A), suggesting T398 phosphorylation of YY1 is critical for 3-HAA-induced apoptosis.

To identify the kinase for the 3-HAA-induced T398 phosphorylation of YY1, the kinase screening assay was performed on the peptide of FAQSTNLK. Three candidate enzymes AKT, mTOR, and PKC了 were chosen from predicted kinases based on the online software (www.cbs.dtu.dk; gps.biocuckoo.cn) (Mok et al.; Xue et al., 2011). Furthermore, proteomics analysis by mass spectrometry following YY1

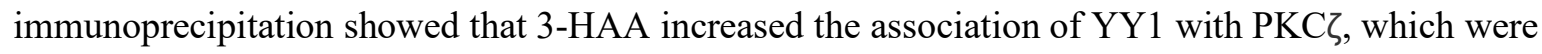
further confirmed by immunoblotting, suggesting 3-HAA recruits PKC $\zeta$ to phosphorylate YY1 (Figs. 5F, S5B \& 5G). The kinase $\mathrm{PKC} \zeta$ significantly increased the peptide phosphorylation, reflected by the autoradiogram on dot blot (Fig. 5H). Also, only the PKC $\zeta$ inhibitor markedly decreased YY1 phosphorylation (Fig. 5I), suggesting PKC $\zeta$ is the kinase for T398phosphorylation of YY1 induced by 3HAA.

Moreover, the PKC $\zeta$ inhibitor markedly decreased the mRNA level and protein level of DUSP6 in the SMMC7721 cells depleted of endogenous YY1 and expressing exogenous wild type YY1, but not in the cells expressing T398A/T398E mutant YY1 (Fig. 5J \& 5K). Eventually, the PKC the level of cleaved Caspase 3 and cleaved PARP in the cells expressing exogenous wild type YY1 but not T398A/T398E mutant YY1 (Fig. 5K). The clinical data that the PKC $\zeta$ expression level is closely correlated with the overall survival of the grade I HCC patients (Fig. S5C), further supports these findings.

Using computational software, we propose a binding model that 3-HAA binds to the site encompassing Gln396 to Thr398 of YY1 (Fig. 5L), which is consistent with our above finding that the Thr398 phosphorylation increasing the YY1 binding to the promoter of DUSP6 gene.

\section{T398 phosphorylation of YY1 is critical for 3-HAA-suppressed HCC xenografts growth}

To further evaluate the effects of T398 phosphorylation of YY1 on 3-HAA-suppressed HCC cell growth, the apoptosis and tumor growth were determined in HCC xenograft mice. In mice with control 
HCC xenografts, 3-HAA (100 mg/kg•day) slowed xenograft growth, leading to smaller tumors, whereas the same dose of 3-HAA had no remarkable effect on YY1 depleting xenograft growth (Fig. 6A). Both the TUNEL assay and flow cytometry revealed a noticeably higher percentage of apoptotic cells only in YY1 expressing HCC xenografts, but not in YY1 depleting HCC xenografts (Fig. 6B-C \& S6A). This result was obtained with SMMC7721 and HepG2 xenografts.

Moreover, in mice with HCC xenograft depleting endogenous YY1 and expressing T398A mutant YY1, 3-HAA (100 mg/kg•day) had no effect on tumor growth. Whereas, the same dose of 3-HAA significantly decreased xenograft growth expressing wild type YY1 (Fig. 6D). The TUNEL assay and flow cytometry also demonstrated a markedly higher percentage of apoptotic cells in xenografts expressing wild type YY1, but not in T398A mutant xenografts (Fig. 6E, 5F \& S6B). In mice with HCC xenografts expressing T398E mutant YY1, the growth of xenografts was obviously slower than the xenografts expressing wild type YY1, and 3-HAA had no effects on these xenograft growth (Fig. 6E). And, the PKC $\zeta$ inhibitor $(0.8 \mathrm{mg} / \mathrm{kg}$.day $)$ treatment restored the growth of xenografts suppressed by 3 HAA (Fig. 6G), and the flow cytometry analysis also displayed a decreased percentage of apoptotic cells in HCC xenografts treated with the PKC $\zeta$ inhibitor, compared to the 3-HAA alone-treated xenografts (Fig. 6H). The GC-MS/MS analysis showed that the 3-HAA content was indeed dramatically increased in 3-HAA-treated xenografts (Fig. 6I). Most importantly, 3-HAA synergizes with IDO inhibitors to suppress HCC xenograft growth (Fig. 6J).

To determine the pharmacokinetic of 3-HAA, the concentration of 3-HAA was analyzed at the time course of administration in the plasma of mice. As shown in Fig. S6D, the half-time of 3-HAA was 3.89 hours in the serum of mice. The concentration of 3-HAA reached $71.3 \mu \mathrm{mol}$ per gram in tumors post 7 days treatment. Moreover, 3-HAA did not disrupt liver/renal function in Balb/c mice at a dose of 800 $\mathrm{mg} / \mathrm{kg}$.day, which was six-fold higher than the tested dose (Fig. S6E). These results highlight the promise of 3-HAA as a potential HCC therapy.

\section{Discussion}

Tryptophan metabolism plays a very important role in development and tumor progression. It not 
only provides critical intermediates for anabolism but also regulates cell signaling. IDO converts tryptophan into kynurenine, which is a well-known functional metabolite of tryptophan and could be further catabolized to 3-HAA. Kynurenine binds AHR to induce immune suppression (tolerance), which is associated with successful embryo implantation but also poor prognosis in various malignancies. The inhibition of IDO1/2 suppresses tumor formation in animal models but failed in stage III of clinical trials. This is probably due to the expression of the other kynurenine generating enzyme TDO, which may not be suppressed by the IDO inhibitor. However, the 3-HAA treatment reversed the tumor-promoting effect of kynurenine and significantly improved the efficacy of IDO1/2 inhibitors on HCC xenografts, suggesting 3-HAA as a negative feedback regulator reverses the tumor cell-hijacked tryptophan metabolism.

The 3-HAA exerts anti-inflammatory and neuroprotective effects by selectively inducing the apoptosis of activated $\mathrm{T}$ cells or suppressing microglia/astrocytes that express cytokines and chemokines (Krause et al., 2011; Lee et al., 2010). The 3-HAA induced the expression of cytoprotective enzyme hemeoxygenase- 1 in astrocytes and microglia, the latter is an enzyme with proven anti-inflammatory and cytoprotective activities (Brusko et al., 2005; Ryter and Choi, 2009). The 3-HAA-induced apoptosis of activated T lymphocytes are linked to oxidative stress and induction of caspases (Hayashi et al., 2007; Hiramatsu et al., 2008). These results indicate that 3-HAA- has a distinct biological function in different type of cells. However, the biological function of 3-HAA largely remains unclear.

In this study, we report that 3-HAA is downregulated in HCC cells and HCC tissues due to the downregulation of KMO and KYNU enzymes as well as the upregulation of the HAAO enzyme, which are regulated by kynurenine signal. The excessive kynurenine generated from tryptophan in HCCs is exported. We also observed that the HCC cell sensitivity to 3-HAA, to some extent, were correlated with the cellular 3-HAA concentration post exogenous 3-HAA treatment. However, the variation of the cellular 3-HAA concentration post exogenous 3-HAA treatment and the endogenous 3-HAA concentration may not completely associate with expression levels of KMO, KYNU, and HAAO enzymes in HCC cells (Fig. 1E \& S2B), since the 3-HAA transporters and drug resistant genes may also involve in 
the regulation of HCC cell sensitivity to 3-HAA. This observation suggests that the heterogeneity of tryptophan metabolism in HCC cells may differ in their sensitivity to 3-HAA.

Moreover, we also discovered that 3-HAA is a ligand of transcription factor YY1 and that binding of the two molecules leads to 3-HAA-mediated alterations in gene expression. 3-HAA up- or downregulated a number of genes via YY1 binding, including DUSP6, IGFBP1, and NROB2 etc. YY1 may bind to two motifs in the DUSP6 promoter; the known consensus binding site, as well as a novel binding site, was identified in this study. The T398 phosphorylation of YY1 may change its structure conformation associating with DNA binding, promoting YY1 binding to its target sequence.

Although the in vitro measurement showed a higher dissociation constant of YY1 with 3-HAA (the KD50 of 3-HAA was $121.7 \mu \mathrm{M}$ ), this may not reflect the binding affinity of YY1 with 3-HAA in cells since the cellular contents of 3-HAA were less than $20 \mu \mathrm{M}$, even in HCC cells treated by 3-HAA or expressing KMO. Moreover, the expression level of solute carriers determines the cellular uptake of 3HAA, thus, the contents of 3-HAA in 3-HAA-treated HCC cells were only 5-10 times more than untreated cells, similar to the level of endogenous 3-HAA in cells expressing KMO (Fig. 1F \& 2B).

Taken together, our results have determined that 3-HAA is a functional metabolite regulating tumor cell fate by binding to and activating the transcription factor YY1 (Fig. 6K). Selective downregulation of 3-HAA by kynurenine signal appears to be essential for HCC growth. Exogenous 3-HAA induces tumor cell apoptosis and inhibits HCC growth, especially improves the efficacy of IDO1/2 inhibitors, suggesting its potential use in HCC therapy. 


\section{References}

Blom, N., Sicheritz-Ponten, T., Gupta, R., Gammeltoft, S., and Brunak, S. (2004). Prediction of posttranslational glycosylation and phosphorylation of proteins from the amino acid sequence. Proteomics 4, 1633-1649.

Brusko, T. M., Wasserfall, C. H., Agarwal, A., Kapturczak, M. H., and Atkinson, M. A. (2005). An integral role for heme oxygenase-1 and carbon monoxide in maintaining peripheral tolerance by CD4+CD25+ regulatory T cells. J Immunol 174, 5181-5186.

Chen, Y., and Guillemin, G. J. (2009). Kynurenine pathway metabolites in humans: disease and healthy States. Int J Tryptophan Res 2, 1-19.

Daub, H., Olsen, J. V., Bairlein, M., Gnad, F., Oppermann, F. S., Korner, R., Greff, Z., Keri, G., Stemmann, O., and Mann, M. (2008). Kinase-selective enrichment enables quantitative phosphoproteomics of the kinome across the cell cycle. Molecular cell 31, 438-448.

Hayashi, T., Mo, J. H., Gong, X., Rossetto, C., Jang, A., Beck, L., Elliott, G. I., Kufareva, I., Abagyan, R., Broide, D. H., et al. (2007). 3-Hydroxyanthranilic acid inhibits PDK1 activation and suppresses experimental asthma by inducing T cell apoptosis. Proceedings of the National Academy of Sciences of the United States of America 104, 18619-18624.

Hiramatsu, R., Hara, T., Akimoto, H., Takikawa, O., Kawabe, T., Isobe, K., and Nagase, F. (2008). Cinnabarinic acid generated from 3-hydroxyanthranilic acid strongly induces apoptosis in thymocytes through the generation of reactive oxygen species and the induction of caspase. J Cell Biochem 103, 4253.

Huynh, H., Soo, K. C., Chow, P. K., Panasci, L., and Tran, E. (2006). Xenografts of human hepatocellular carcinoma: a useful model for testing drugs. Clin Cancer Res 12, 4306-4314.

Kaludov, N. K., Bowman, T. L., Sikorski, E. M., and Hurt, M. M. (1996). Cell cycle-regulated binding of nuclear proteins to elements within a mouse H3.2 histone gene. Proceedings of the National Academy of Sciences of the United States of America 93, 4465-4470.

Khan, A., Fornes, O., Stigliani, A., Gheorghe, M., Castro-Mondragon, J. A., van der Lee, R., Bessy, A., Cheneby, J., Kulkarni, S. R., Tan, G., et al. (2018a). JASPAR 2018: update of the open-access database of transcription factor binding profiles and its web framework. Nucleic Acids Res 46, D1284.

Khan, A., Fornes, O., Stigliani, A., Gheorghe, M., Castro-Mondragon, J. A., van der Lee, R., Bessy, A., Cheneby, J., Kulkarni, S. R., Tan, G., et al. (2018b). JASPAR 2018: update of the open-access database of transcription factor binding profiles and its web framework. Nucleic Acids Res 46, D260-D266.

Kim, J., and Kim, J. (2009). YY1's longer DNA-binding motifs. Genomics 93, 152-158.

Krause, D., Suh, H. S., Tarassishin, L., Cui, Q. L., Durafourt, B. A., Choi, N., Bauman, A., Cosenza-Nashat, M., Antel, J. P., Zhao, M. L., and Lee, S. C. (2011). The tryptophan metabolite 3-hydroxyanthranilic acid plays anti-inflammatory and neuroprotective roles during inflammation: role of hemeoxygenase- 1 . The American journal of pathology 179, 1360-1372.

Lee, S. M., Lee, Y. S., Choi, J. H., Park, S. G., Choi, I. W., Joo, Y. D., Lee, W. S., Lee, J. N., Choi, I., and Seo, S. K. (2010). Tryptophan metabolite 3-hydroxyanthranilic acid selectively induces activated T cell death via intracellular GSH depletion. Immunol Lett 132, 53-60.

Li, Y., Innocentin, S., Withers, D. R., Roberts, N. A., Gallagher, A. R., Grigorieva, E. F., Wilhelm, C., and Veldhoen, M. (2011). Exogenous stimuli maintain intraepithelial lymphocytes via aryl hydrocarbon receptor activation. Cell 147, 629-640.

Llovet JM1, R. S., Mazzaferro V, Hilgard P, Gane E, Blanc JF, de Oliveira AC, Santoro A, Raoul JL, Forner A, Schwartz M, Porta C, Zeuzem S, Bolondi L, Greten TF, Galle PR, Seitz JF, Borbath I, Häussinger D, Giannaris T, Shan M, Moscovici M, Voliotis D, Bruix J; SHARP Investigators Study Group. (2008). Sorafenib in advanced hepatocellular carcinoma. N Engl J Med 359, 378-390. 
Mok, J., Kim, P. M., Lam, H. Y., Piccirillo, S., Zhou, X., Jeschke, G. R., Sheridan, D. L., Parker, S. A., Desai, V., Jwa, M., et al. (2010). Deciphering protein kinase specificity through large-scale analysis of yeast phosphorylation site motifs. Science signaling 3, ra12.

Morita, T., Saito, K., Takemura, M., Maekawa, N., Fujigaki, S., Fujii, H., Wada, H., Takeuchi, S., Noma, A., and Seishima, M. (2001). 3-Hydroxyanthranilic acid, an L-tryptophan metabolite, induces apoptosis in monocyte-derived cells stimulated by interferon-gamma. Ann Clin Biochem 38, 242-251.

Opitz, C. A., Litzenburger, U. M., Sahm, F., Ott, M., Tritschler, I., Trump, S., Schumacher, T., Jestaedt, L., Schrenk, D., Weller, M., et al. (2011). An endogenous tumour-promoting ligand of the human aryl hydrocarbon receptor. Nature 478, 197-203.

Piscianz, E., Cuzzoni, E., De ludicibus, S., Valencic, E., Decorti, G., and Tommasini, A. (2011). Differential action of 3-hydroxyanthranilic acid on viability and activation of stimulated lymphocytes. Int Immunopharmacol 11, 2242-2245.

Piya, S., Kim, J. Y., Bae, J., Seol, D. W., Moon, A. R., and Kim, T. H. (2012). DUSP6 is a novel transcriptional target of p53 and regulates p53-mediated apoptosis by modulating expression levels of Bcl-2 family proteins. Febs Lett 586, 4233-4240.

Ryter, S. W., and Choi, A. M. (2009). Heme oxygenase-1/carbon monoxide: from metabolism to molecular therapy. Am J Respir Cell Mol Biol 41, 251-260.

Schrocksnadel, K., Wirleitner, B., Winkler, C., and Fuchs, D. (2006). Monitoring tryptophan metabolism in chronic immune activation. Clin Chim Acta 364, 82-90.

Schwarcz, R., Bruno, J. P., Muchowski, P. J., and Wu, H. Q. (2012). Kynurenines in the mammalian brain: when physiology meets pathology. Nat Rev Neurosci 13, 465-477.

Sharma, M. D., Baban, B., Chandler, P., Hou, D. Y., Singh, N., Yagita, H., Azuma, M., Blazar, B. R., Mellor, A. L., and Munn, D. H. (2007). Plasmacytoid dendritic cells from mouse tumor-draining lymph nodes directly activate mature Tregs via indoleamine 2,3-dioxygenase. J Clin Invest 117, 2570-2582.

Song, C., Ye, M., Liu, Z., Cheng, H., Jiang, X., Han, G., Songyang, Z., Tan, Y., Wang, H., Ren, J., et al. (2012). Systematic analysis of protein phosphorylation networks from phosphoproteomic data. Mol Cell Proteomics 11, 1070-1083.

Torre, L. A., Bray, F., Siegel, R. L., Ferlay, J., Lortet-Tieulent, J., and Jemal, A. (2015). Global cancer statistics, 2012. CA: a cancer journal for clinicians 65, 87-108.

Xue, Y., Liu, Z., Cao, J., Ma, Q., Gao, X., Wang, Q., Jin, C., Zhou, Y., Wen, L., and Ren, J. (2011). GPS 2.1: enhanced prediction of kinase-specific phosphorylation sites with an algorithm of motif length selection. Protein Eng Des Sel 24, 255-260.

Yevshin, I., Sharipov, R., Kolmykov, S., Kondrakhin, Y., and Kolpakov, F. (2019). GTRD: a database on gene transcription regulation-2019 update. Nucleic Acids Res 47, D100-D105. 


\section{Acknowledgments}

We thank Profs. Lei Shen (Shanghai Jiao Tong University School of Medicine), Qunying Lei (Fudan University School of Medicine) and Chief Editor Dangsheng Li (Journal of Cell Research) for useful discussions. This study was supported by grants from the Ministry of Science and Technology of the People's Republic of China (2018YFC1313205), the Shanghai Committee of Science and Technology (11DZ2260200) and the National Natural Science Foundation of China (81572300) (81872342) to Dr. Mi.

\section{Author contributions}

G.G., Z.S., S.L. and X.X. performed most of experiments; Y.Y., J.Z. and B.B. performed some of animal experiments; X.G. performed metabolites analysis, P.X. and W.Z. synthesize the 3HAA derivatives; N.Z. and A.Z. performed the 3-HAA and YY1 binding experiments; W.L. performed ChIP-Seq analysis and kinase screening; J.M. initiated the project, led the project team, designed experiments, analyzed results and wrote the paper with input from all authors.

\section{Conflict of interest}

No conflict of interest to declare. 


\section{Figure legends}

Figure 1 3-hydroxyanthranilic acid (3-HAA) is selectively decreased in hepatocellular carcinoma

A. Quantitative analysis of tryptophan metabolites by LC-MS/MS and GC-MS in HCC and corresponding paratumor tissues.

B. Quantitative analysis of tryptophan metabolites by LC-MS/MS and GC-MS in normal hepatic cells and HCC cells.

C. Immunohistochemistry staining of 3-HAA in clinical HCC samples.

D. Metabolic flux analysis of tryptophan metabolites in Huh7 and HepG2 cells. L-Tryptophan was completely ${ }^{13} \mathrm{C}$-labelled. The content of tryptophan catabolites in cells and medium was assessed using LC-MS/MS. The M0 stands for no carbon in tryptophan was ${ }^{13} \mathrm{C}$-labelled, M11 stands for all 11 carbon in tryptophan were ${ }^{13} \mathrm{C}$-labelled.

E. Expression analysis of metabolic enzymes involved in 3-HAA generation.

F. The effect of KMO overexpression on the concentration of endogenous 3-HAA and tryptophan catabolites in SMMC7721 cells.

Figure 2 3-hydroxyanthranilic acid (3-HAA) induces apoptosis of hepatocellular carcinoma cells

A. Analysis of the growth of HCC cells treated for 4 days with one of four tryptophan metabolites (100 $\mu \mathrm{M})$.

B. Gene Ontology (GO) enrichment analysis of HCC SMMC7721 cells treated by 3-HAA for 8 hours.

C. Apoptosis analysis by TUNEL assay in SMMC7721 cells treated for $24 \mathrm{~h}$ with 3-HAA at the indicated dose.

D. Analysis of apoptosis signaling in HCC cells treated with $100 \mu \mathrm{M} 3-\mathrm{HAA}$ for indicated time or treated for $12 \mathrm{~h}$ with 3 -HAA at the indicated dose.

E. The effects of three types of inhibitors on 3-HAA-induced HCC cell death. ZVAD: apoptosis inhibitor $(20 \mu \mathrm{M})$; Nec1: necrosis inhibitor $(100 \mu \mathrm{M})$; 3-MA: autophagy inhibitor $(5 \mathrm{mM})$. The dose of 3-HAA was $100 \mu \mathrm{M}$. Cells were treated for 4 days. 
F and G. Apoptosis analysis by TUNEL assay and flow cytometry in SMMC7721 xenografts with and without 3-HAA treatment. The dose of 3-HAA was $100 \mathrm{mg} / \mathrm{kg} \bullet$ day. Mice were treated for 7 days and sacrificed on day 10 .

H. The influence of endogenous 3-HAA on cell growth and apoptosis signaling of HCC cells. The KMO was overexpressed in SMMC 7721. The ${ }^{* *}: \mathrm{p}<0.01$. The concentration of 3-HAA and ZVAD was 100 $\mu \mathrm{M}$ and $20 \mu \mathrm{M}$, respectively.

I. Tumor formation analysis of HCC cells overexpressing KMO. The effect of endogenous 3-HAA on xenografted tumor formation.

J. The effect of KMO overexpression on apoptosis of SMMC7721 cells.

$\mathrm{K}$. The effect of KMO expression on the disease-free survival of HCC patients. The patients were divided by the median of KMO expression value.

\section{Figure 3 3-HAA induces tumor cell apoptosis by upregulating DUSP6 expression}

A. The Heatmap of the top consistently upregulated genes in SMMC7721, Hep3B, and SMMC97H. Deep sequencing was used to profile gene expression in HCC SMMC7721, Hep3B, and SMMC97H cells. HCC cells were treated with $100 \mu \mathrm{M} 3$-HAA for 1,8 , or $24 \mathrm{~h}$.

B. Top consistently upregulated genes were individually verified using quantitative PCR and immunoblotting.

C. DUSP6 expression reversely correlated with overall survival of HCC patients, as analyzed by the K-M plotter. The total patient number was 415 . The HCC patients were divided by the median of KMO expression value.

D. Effects of DUSP6 knockdown on the growth of HCC cells. Cells were treated with $100 \mu \mathrm{M} 3-\mathrm{HAA}$ for the indicated time. DUSP6 was stably knocked down in SMMC7721 cells using lentivirus-generated shRNA.

E. Effects of DUSP6 knockdown on HCC cell apoptosis. SMMC7721 cells were treated with $100 \mu \mathrm{M}$ of 3-HAA for $12 \mathrm{~h}$, stained for Annexin V, and analyzed by flow cytometry. 
F. Effects of DUSP6 knockdown on 3-HAA-activated apoptosis signaling. SMMC7721 cells depleted of DUSP6 were treated with $100 \mu \mathrm{M} 3$-HAA for $24 \mathrm{~h}$.

G. DUSP6 knockdown recovered 3-HAA-suppressed xenograft growth. Animals were treated with 3HAA (100 mg/kg•day) for seven days, then sacrificed on day 10. SMMC7721 cells were subcutaneously injected into mice. The middle graph shows xenograft weights in different groups. Photographs on the right show representative tumors in different groups.

Figure 4 3-HAA upregulated DUSP6 expression by binding with transcription factor YY1

A. The transcription factors potentially binding with promoters of top 4 genes upregulated by 3-HAA.

B. The common proteins between 3-HAA-increased chromatin binding proteins and predicted transcription factors binding to the DUSP6 promoter region.

C. 3-HAA increases YY1 nuclear translocation. SMMC7721 cells were treated with $100 \mu \mathrm{M}$ of 3-HAA for 8 hours.

D. YY1 knockdown abolished 3-HAA-induced DUSP6 expression. SMMC7721 cells were treated with $100 \mu \mathrm{M}$ of 3-HAA for the indicated time.

E. The effect of YY1 knockdown on 3-HAA-activated apoptosis signaling. The concentration of 3-HAA was $100 \mu \mathrm{M}$.

F. The effect of YY1 knockdown on 3-HAA-suppressed HCC cell growth. The concentration of 3-HAA was $100 \mu \mathrm{M}$. The **: $\mathrm{P}<0.01$.

G. The effect of YY1 knockdown on 3-HAA-induced HCC apoptosis, analyzed by flow cytometry. The concentration of 3 -HAA was $100 \mu \mathrm{M}$. The **: $\mathrm{P}<0.01$.

H. Transcription activity of YY1 on the DUSP6 promoter, as determined in a luciferase reporter assay.

The schematic depicts the plasmid encoding luciferase under the control of the DUSP6 promoter, which was truncated or mutated as indicated.

I. 3-HAA binding to YY1 determined by electrophoretic mobility shift assay (EMSA). The synthesized oligonucleotide was labeled at the 5' terminus with FAM, and YY1 was purified using a Hitrap heparin column. 
J. 3-HAA increased YY1 enrichment on the DUSP6 promoter, analyzed by chromatin immunoprecipitation (ChIP) and quantitative PCR (QPCR). The dose of 3-HAA was $100 \mu \mathrm{M}$.

K. The ChIP-sequencing analysis of YY1 on the DUSP6 gene. The HCC cells were treated with the indicated dose of 3-HAA prior to ChIP-sequencing.

L. NMR measurement of direct binding between 3-HAA and YY1. T1r NMR spectra for $6 \mathrm{~b}$ (red) alone or in the presence of SPOP at $2.5 \mathrm{mM}$ (blue), $5 \mathrm{mM}$ (cyan), or $10 \mathrm{mM}$ (green). The STD spectrum for $6 \mathrm{~b}$ was recorded in the presence of $5 \mathrm{mM}$ SPOP.

M. 3-HAA bound YY1 protein as shown by surface plasmon resonance. Graphs of equilibrium unit response versus concentrations are shown. The estimated $K_{\mathrm{d}}$ was $121.7 \mu \mathrm{M}$.

\section{Figure $5 \mathrm{PKC} \zeta$ phosphorylates YY1 at Thr 398 in response to 3-HAA}

A. The YY1 phosphorylation was analyzed by the immunoblotting and the mass-spectrometry. The YY1 was blotted on the enriched phospho-proteins from SMMC7721 cells. The YY1 modification was analyzed by the mass-spectrometry following YY1 immunoprecipitation.

B. The YY1 mutation of T398A attenuates the 3-HAA-increased YY1 nuclear accumulation. The concentration of 3-HAA was $100 \mu \mathrm{M}$ and treated for $24 \mathrm{~h}$.

C. The T398A but not S247A mutation abolished 3-HAA-induced YY1 phosphorylation. The YY1 was conjugated with HA tag. The YY1 phosphorylation was detected by the T398 phospho-specific antibody.

D. The mutation of T398E in YY1 promoted DUSP6 expression, whereas the T398A mutation suppressed DUSP6 upregulation. The YY1 were fused with HA tag.

E. The YY1 mutation of T398A reduces the 3-HAA-induced apoptosis, analyzed by the TUNEL assay.

F. 3-HAA increased PKC $\zeta$ binding to YY1, analyzed by co-immunoprecipitation and mass-spec.

G. 3-HAA increased PKC $\zeta$ binding to YY1, analyzed by immunoblots.

H. The kinase screening for T398 phosphorylation of YY1. The kinase candidates were predicted by online tools NetPhos 3.1 (www.cbs.dtu.dk/services/NetPhos) (Blom et al., 2004) and GPS 5.0 (gps.biocuckoo.cn)(Song et al., 2012). 
I. The effect of kinase inhibitors on 3-HAA-induced YY1 phosphorylation. The YY1 phosphorylation was detected by the T398 phospho-specific antibody. The concentration of 3-HAA was $100 \mu \mathrm{M}$. AKT inhibitor, MK2206 (0.5 $\mu \mathrm{M})$; PKC inhibitor, Go6983 (0.5 $\mu \mathrm{M})$; mTOR inhibitor, rapamycin (0.1 $\mu \mathrm{M})$. L. The proposed 3-HAA binding model with YY1.

\section{Figure 6 T398 phosphorylation of YY1 is critical for 3-HAA-suppressed HCC xenografts growth}

A. YY1 knockdown recovered the 3-HAA-inhibited HCC xenografts growth.

B. \& C. The apoptosis analysis in xenografts by TUNEL assay and the flow cytometry. The histogram presents as mean $\pm \mathrm{SD}(*: \mathrm{P}<0.05 ; * *: \mathrm{P}<0.01)$.

D. The T398A mutation of YY1 abolished the 3-HAA-inhibited HCC xenografts growth.

E. \& F. The apoptosis analysis in xenografts by TUNEL assay and the flow cytometry. The histogram presents as mean $\pm \mathrm{SD}(*: \mathrm{P}<0.05 ; * *: \mathrm{P}<0.01$.).

G. The PKC $\zeta$ inhibitor reverted the HCC xenografts growth inhibited by 3-HAA.

H. The apoptosis analysis in xenografts by the flow cytometry. The histogram presents as mean $\pm \mathrm{SD}(*$ : $\mathrm{P}<0.05 ; * *: \mathrm{P}<0.01$.

I. The concentration of 3-HAA in tumors post-administration. The 3-HAA content in xenografts was detected by LC-MS/MS.

J. 3-HAA synergizes with IDO inhibitors to HCC xenograft growth. The dose of both 3-HAA and Epacadostat (IDO inhibitor) were $100 \mathrm{mg} / \mathrm{kg}$.day, respectively.

K. The working model of 3-HAA-inducing apoptosis.

Note: The mouse xenografts were generated by the inoculation of $1.5 \times 10^{\wedge} 6$ of SMMC7721 cells into the armpit of rear limb. The 3-HAA (100 mg/kg.day) was administered by intraperitoneal injection for 7 days. Tumor volumes are presented as mean $\pm \mathrm{SD}(*$ : $\mathrm{P}<0.05 ; * *: \mathrm{P}<0.01$.). Photographs on the right show representative xenografts in different groups. 


\section{STAR Methods}

\section{Metabolism flux analysis by LC-MS/MS}

For the flux experiment of tryptophan catabolism, tryptophan in medium was replaced by fully ${ }^{13} \mathrm{C}-$ labeled tryptophan (tryptophan- ${ }^{13} \mathrm{C}_{11}$ ). Sample preparation and LC-MS/MS analysis were the same as for quantitation of tryptophan catabolic products, except that the parameters of MRM transitions were different. MRM transitions for catabolic products of tryptophan $-{ }^{13} \mathrm{C}_{11}(\mathrm{M} 11: 320>273)$ were set as 5hydroxyindoleacetic acid (M10: 323 > 155), kynurenine (M10: 427 > 122), kynurenic acid (M10: $304>$ 105), 3-hydroxykynurenine (M10: $547>05)$, xanthurenic acid (M10: 424 > 05), 3-hydroxyanthranilic acid (M7: 369 > 247), cinnabarinic acid (M14: $419>105)$. Simultaneously, the MRM transitions (M0) of unlabeled tryptophan and its catabolic products were acquired using the same settings as in quantitation experiments.

\section{Quantitative proteomics}

Cell samples were sonicated three times on ice using a high-intensity ultrasonic processor (Scientz, Ninbo, Zhejiang, China) in lysis buffer ( $8 \mathrm{M}$ urea, $1 \%$ Protease Inhibitor Cocktail). The supernatant was collected and proteins were reduced with $5 \mathrm{mM}$ dithiothreitol for $30 \mathrm{~min}$ at $56^{\circ} \mathrm{C}$, then alkylated with 11 $\mathrm{mM}$ iodoacetamide for $15 \mathrm{~min}$ at room temperature in darkness. Following addition of $100 \mathrm{mM}$ TEAB to dilute the urea to $<2 \mathrm{M}$, trypsin was added to the protein samples first at a trypsin-to-protein mass ratio of 1:50 for digestion overnight, then at a ratio of 1:100 for a second digestion lasting $4 \mathrm{~h}$.

After trypsin digestion, peptides were desalted on a Strata X C18 SPE column (Phenomenex) and vacuum-dried. Peptides were reconstituted in $0.5 \mathrm{M}$ TEAB and processed according to the manufacturer's protocol for TMT kit/iTRAQ kit. The tryptic peptides dissolved in $0.1 \%$ formic acid (solvent A) were directly loaded onto a custom-made reverse-phase analytical column (15-cm length, $75 \mu \mathrm{m}$ i.d.) on an EASY-nLC 1000 UPLC system. The gradient to solvent B ( $0.1 \%$ formic acid in $98 \%$ acetonitrile) increased from $6 \%$ to $23 \%$ over $26 \mathrm{~min}$, then from $23 \%$ to $35 \%$ in $8 \mathrm{~min}$ and then to $80 \%$ in $3 \mathrm{~min}$, after which it remained at $80 \%$ for $3 \mathrm{~min}$. The flow rate was constant at $400 \mathrm{~nL} / \mathrm{min}$.

Peptides were subjected to NSI source followed by tandem mass spectrometry (MS/MS) in Orbitrap 
Fusion $^{\mathrm{TM}}$ Tribrid $^{\mathrm{TM}}$ (Thermo, CA, USA) coupled online to the UPLC. Intact peptides were detected in the Orbitrap at a resolution of 60,000. Peptides were selected for MS/MS by NCE at 35; ion fragments were detected in the Orbitrap at a resolution of 30,000. A data-dependent procedure that alternated between one MS scan followed by $10 \mathrm{MS} / \mathrm{MS}$ scans was applied for the top 10 precursor ions above a threshold intensity, which were greater than $5 \times 10^{3}$ in the MS survey scan with dynamic exclusion of $30.0 \mathrm{~s}$. The electrospray voltage applied was $2.0 \mathrm{kV}$. Automatic gain control was used to prevent the orbitrap from overfilling; $5 \times 10^{4}$ ions were accumulated to generate MS/MS spectra. For MS scans, the $\mathrm{m} / z$ scan range was from 350 to 1550 . The fixed first mass was set as $100 \mathrm{~m} / \mathrm{z}$. MS/MS data were processed using the Maxquant search engine (version 1.5.2.8). Carbamidomethyl on cysteine was specified as a fixed modification, while oxidation on methionine was specified as a variable modification. FDR was adjusted to $<1 \%$ and the minimum score for peptides was set to $>40$.

\section{NMR detection of 3-HAA-YY1 interaction}

NMR experiments based on the Carr-Purcell-Meiboom-Gill (CPMG) sequence and saturation transfer difference (STD) were applied to investigate 3-HAA-YY1 interactions. All NMR spectra were acquired at $25{ }^{\circ} \mathrm{C}$ on a $600 \mathrm{MHz}$ Bruker Avance III spectrometer equipped with a cryogenically cooled probe (Bruker Biospin, Germany). Samples containing $200 \mu \mathrm{M} 3$-HAA and $200 \mu \mathrm{M} 3-\mathrm{HAA}$ in the presence of $5 \mu \mathrm{M}$ protein were dissolved in Tris- $\mathrm{HCl}$ buffer $(50 \mathrm{mM}$ Tris- $\mathrm{HCl}, \mathrm{pH} \mathrm{7.4,} 100 \mathrm{mM} \mathrm{NaCl}$, $5 \%$ DMSO, $95 \% \mathrm{D}_{2} \mathrm{O}$ ) and then used in NMR data acquisition. CPMGs were recorded using the pulse sequence of solvent-suppressed $1 \mathrm{D}{ }^{1} \mathrm{H} \mathrm{CPMG}$ (cpmgPrld). The $90^{\circ}$ pulse length was adjusted to approximately $11.82 \mu \mathrm{s}$. A total of 4 dummy scans and 64 free induction decays (FIDs) were collected into $13 \mathrm{~K}$ acquisition points, covering a spectral width of $8 \mathrm{kHz}(13.3 \mathrm{ppm})$ and giving an acquisition time of $3 \mathrm{~s}$. STD data were acquired using 4 dummy scans and a relaxation delay of $3 \mathrm{~s}$, followed by $40-\mathrm{dB}$ pulsed irradiation at a frequency of $-1.0 \mathrm{ppm}$ or alternatively $33 \mathrm{ppm}$. Total time to acquire the STD spectrum was 21 min with 128 FIDs.

\section{Surface plasmon resonance and microscale thermophoresis}


To measure the affinity of 3-HAA to YY1 protein, the dissociation constants were measured using a BIAcore T200 instrument (GE Healthcare, CA, USA) with a CM5 sensor chip (GE Healthcare). The YY1 protein was immobilized on a CM5 sensor chip in sodium acetate buffer ( $1 \mu \mathrm{g} / \mathrm{mL}, \mathrm{pH} 5.5)$, and 3-HAA was gradually titrated at the indicated concentrations. The 3-HAA was injected at a flow rate of 30 $\mu \mathrm{L} / \mathrm{min}$. The association time was $120 \mathrm{~s}$ and the dissociation time was $420 \mathrm{~s}$. The binding constant was calculated using a 1:1 Langmuir binding model via the BIAevaluation software.

The dissociation constant of 3-HAA for YY1 was also measured by microscale thermophoresis assay according to manufacturer's protocol (Nanotemper Technologies Ltd., Shanghai, China). YY1 protein was labeled using NT650-NHS labeling kit (Nanotemper Technologies Ltd., Shanghai, China), and the concentration of labeled YY1 protein was set to $50 \mathrm{nM}$. The unlabelled 3-HAA was gradually diluted from $2500 \mu \mathrm{M}$ to $76.3 \mathrm{nM}$ as indicated. YY1 and 3-HAA interacted in PBS buffer containing 2.5\% DMSO, $0.05 \%$ Tween-20, 85mM NaCl. Measurements were made using the Monolith NT.115 (NanoTemper), and data were analyzed using MO.Affinity analysis $(\times 64)$ software. Graphs were blotted using Prism 5 software.

\section{TUNEL assay}

The cover glass was placed in the 24-well plate, and the HCC cells were inoculated on the cover glass overnight. DMSO or $100 \mu \mathrm{M} 3$-HAA was added to culture medium for 48 hours. The cells were washed with PBS for 3 times. Add $0.5 \mathrm{~mL}$ of $4 \%$ paraformaldehyde and fix cells at room temperature for 10 minutes. Cells were treated with $0.4 \%$ Triton X-100 for 5 minutes and washed with PBS. Cells on the cover glass were treated with TUNEL staining solution and incubated in a wet box at $37^{\circ} \mathrm{C}$ for 1 hour. DAPI staining solution was used to stain the nuclear for 5 minutes in dark. Cells were observed and photographed under a fluorescence microscope.

\section{Xenograft HCC mouse model}


Six-week-old male BALB/c mice were purchased form Lingchang, Shanghai, China. Xenograft mouse model of HCC was generated by injecting SMMC-7721 HCC cells $\left(1.5 \times 10^{\wedge} 6\right)$ subcutaneously into the armpit of rear limb. Mice were injected intraperitoneally with $100 \mathrm{mg} / \mathrm{kg} 3-\mathrm{HAA}$ or equal amount of DMSO every day. when tumors reaching $1 \mathrm{~cm}^{3}$, the PKC inhibitor Go6983 was injected intravenously every two days and the dose of PKC inhibitor was administered at the dose of $0.8 \mathrm{mg} / \mathrm{kg}$.day.

After two weeks, subcutaneous transplanted tumors were removed, and the volume were measured and the tumors were photographed. Following homogenation or tissue slicing, the flow cytometry analysis and TUNEL assay were performed to determine the ratio of apoptotic cells in xenografts. For flow cytometry analysis, cells were collected in binding buffer and stained with Annexin V-APC and PI, and apoptotic signals were detected by flow cytometry. 
A

Figure 1
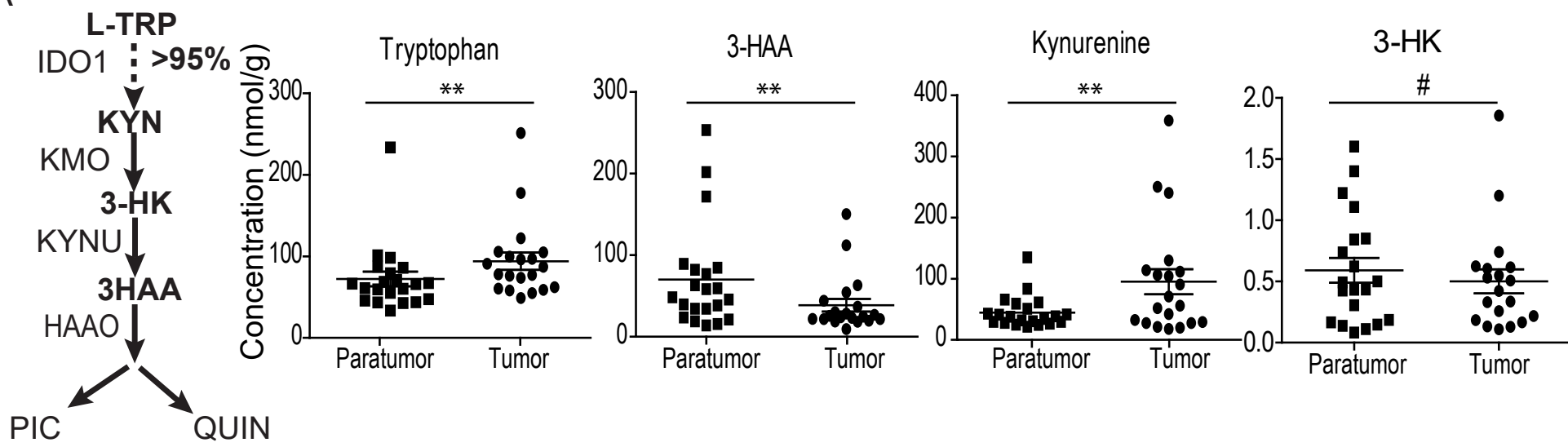

B

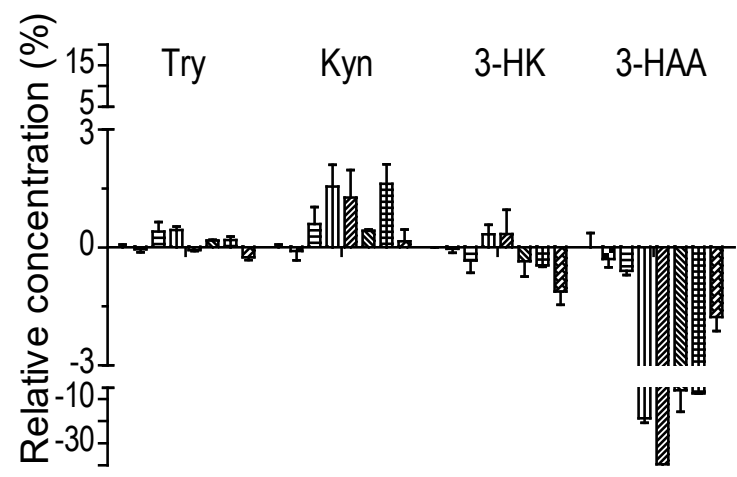

요믈

m HepG2

回 SMMC7721

m SMMC97H

SMMC97L

PLC8024

皿 Hep3B

Huh7
C

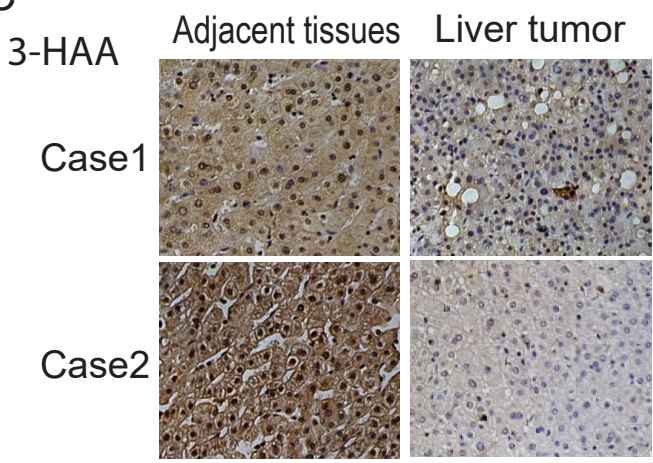

D

Cellular

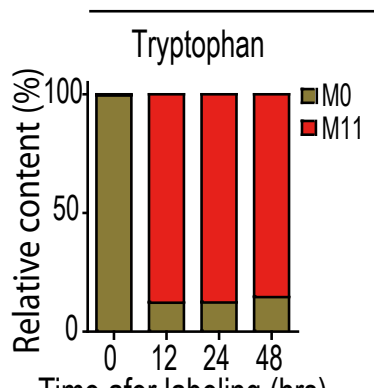

Time afer labeling (hrs)

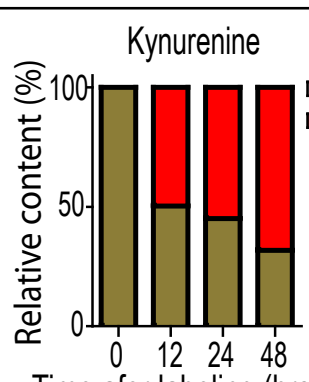

Time afer labeling (hrs)

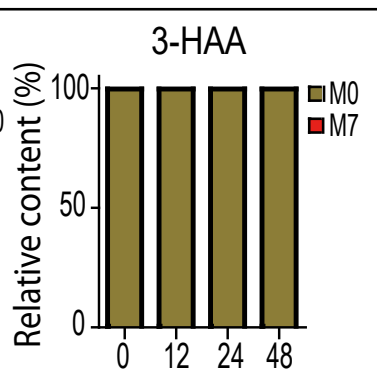

Time afer labeling (hrs)

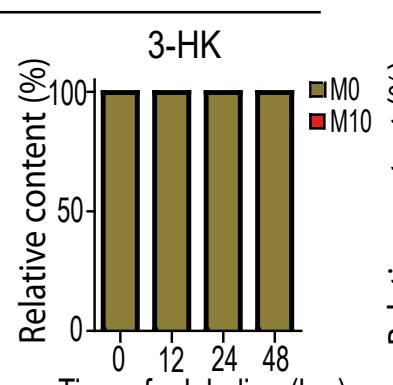

Time afer labeling (hrs)

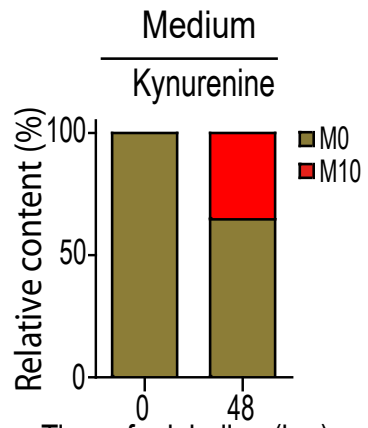

Time afer labeling (hrs)

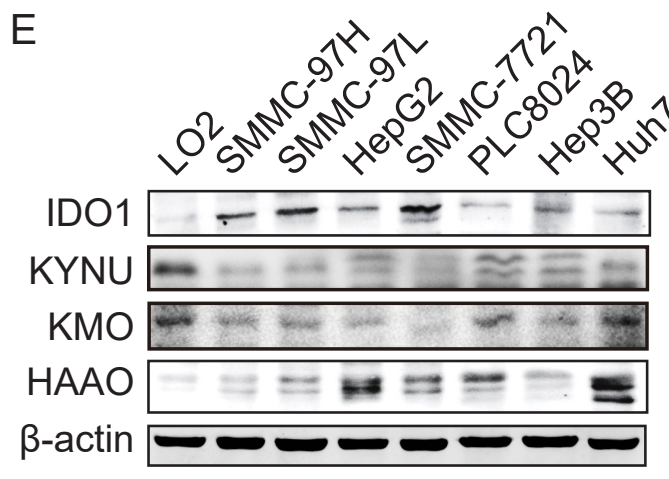

F

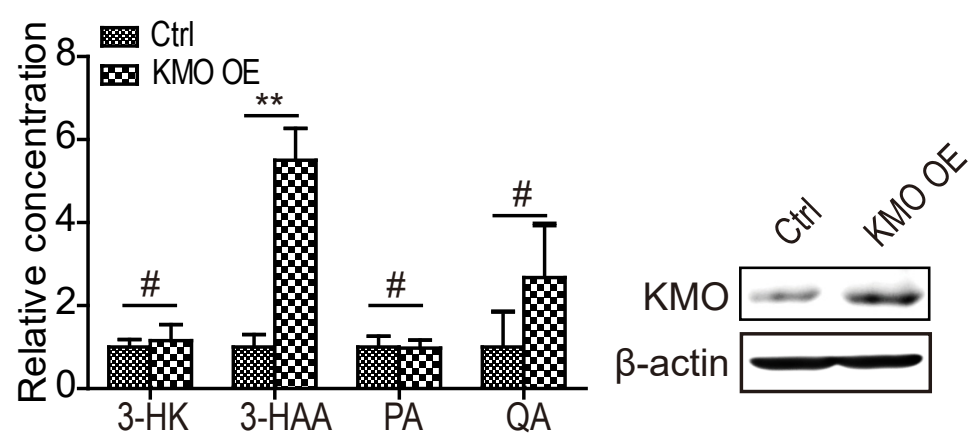


A

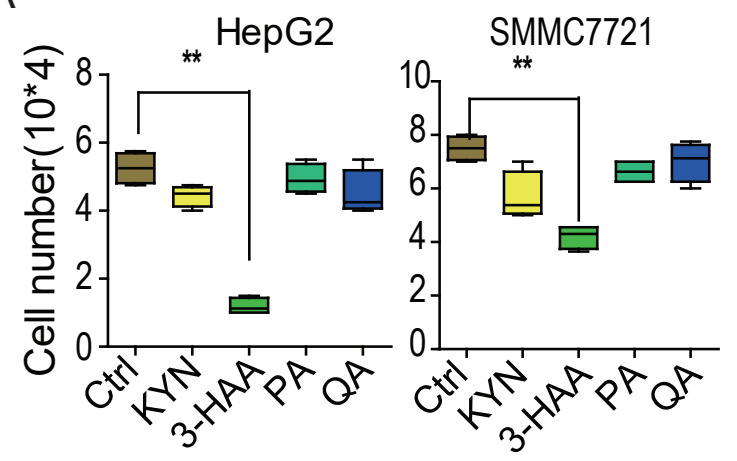

C

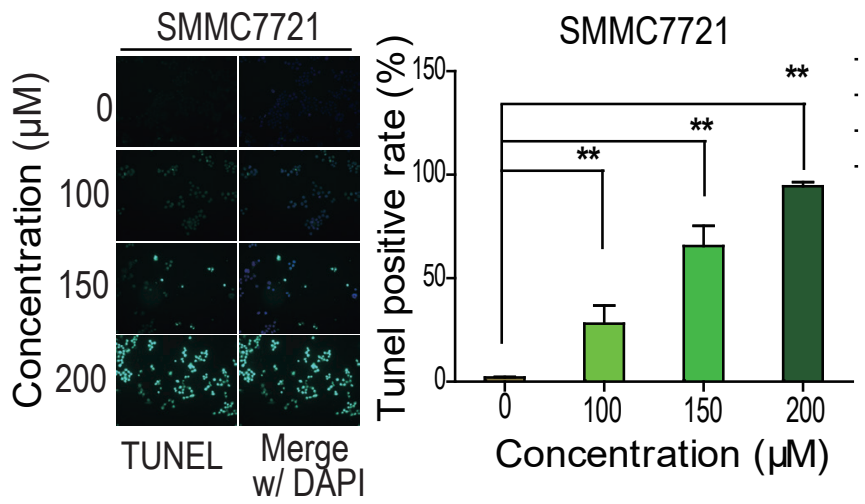

E
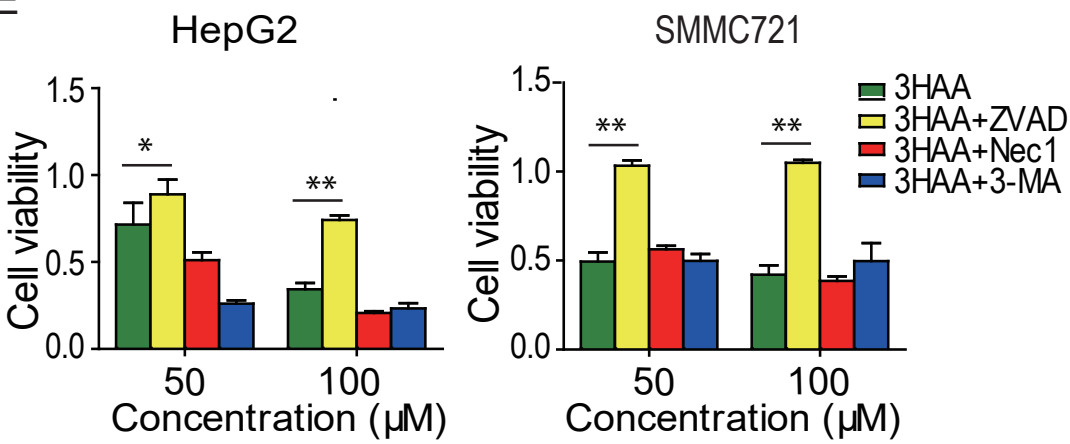

B

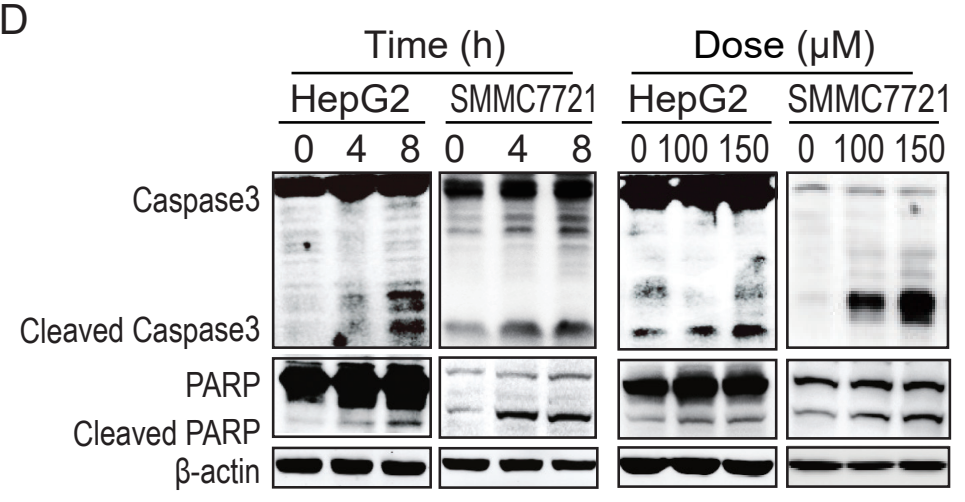

F

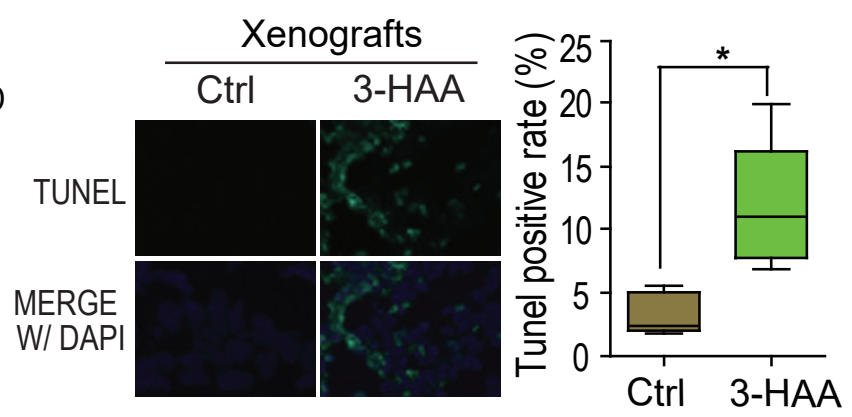

G

Ctrl

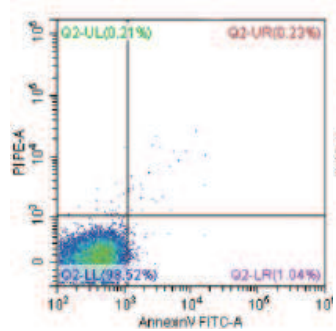

I

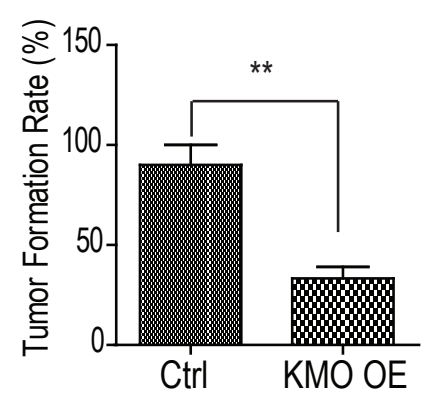

3-HAA (100mg/kg.day)

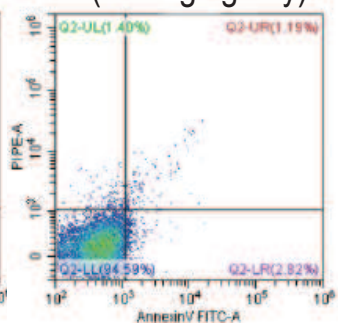

J

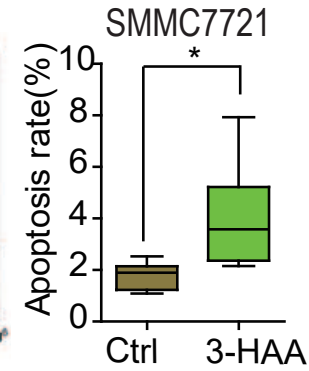

Ctrl 3-HAA

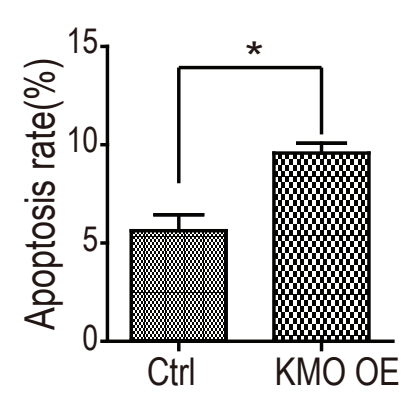

$\mathrm{H}$
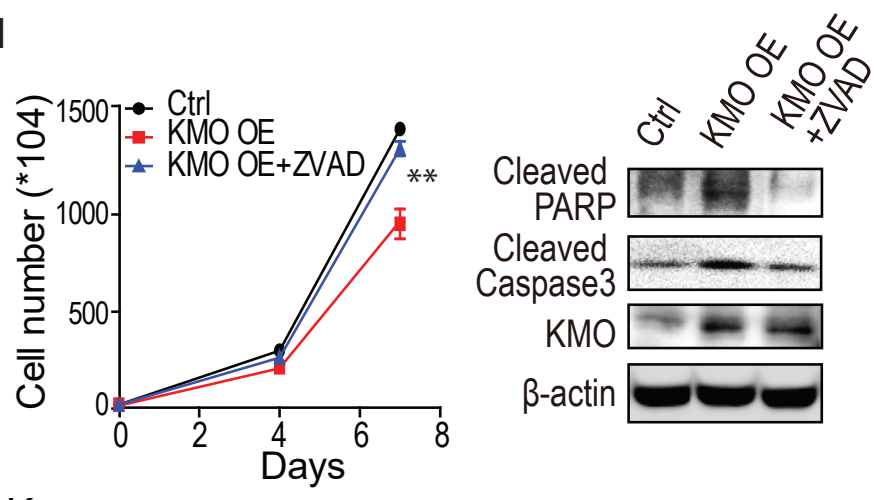

K

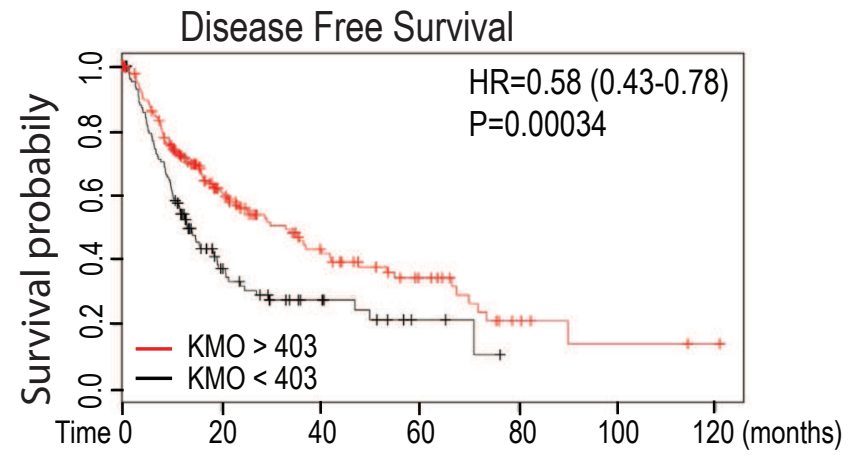


A

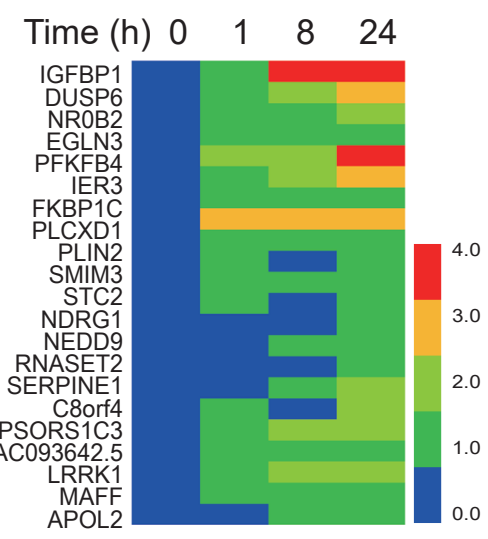

B

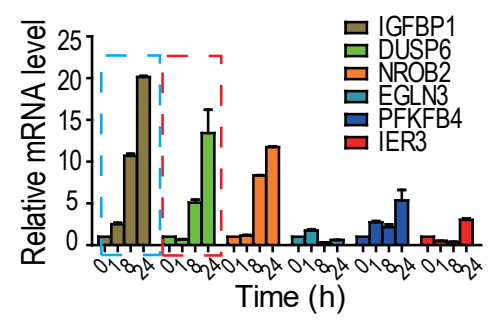

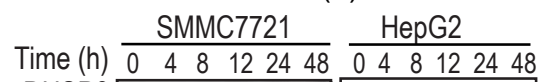
DUSP6 $-\ldots-0-1-\cdots$ IGFBP1 $-\cdots-\infty$ $\beta$-actin -1-n-a
C

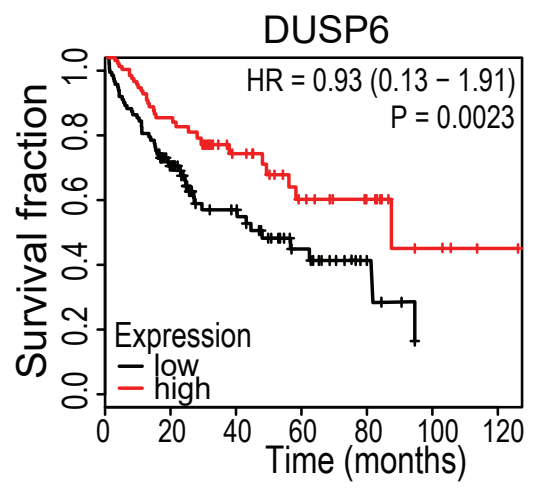

D

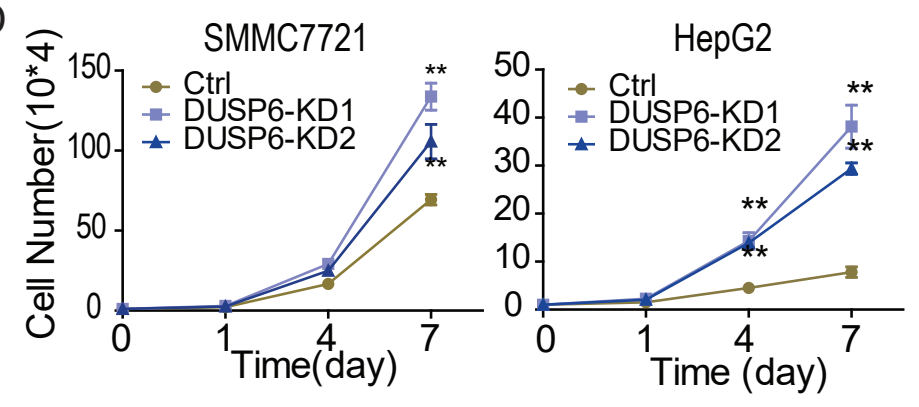

F

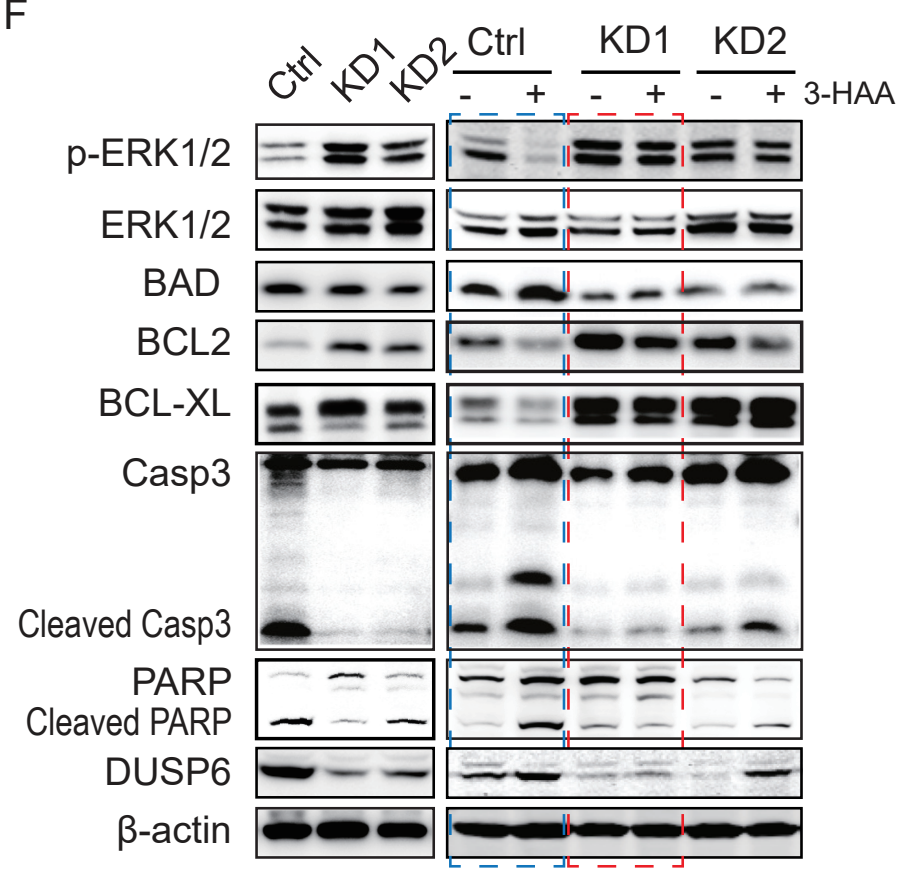

G

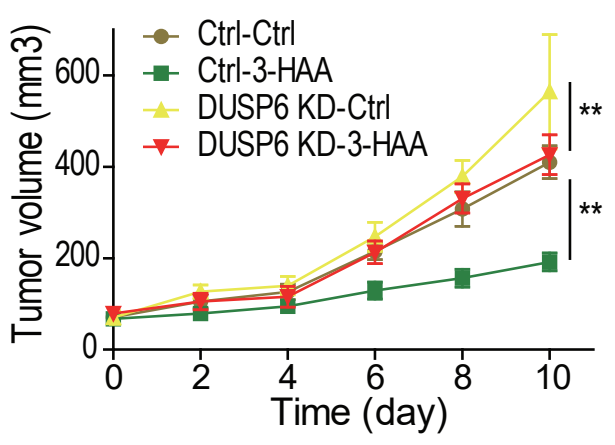

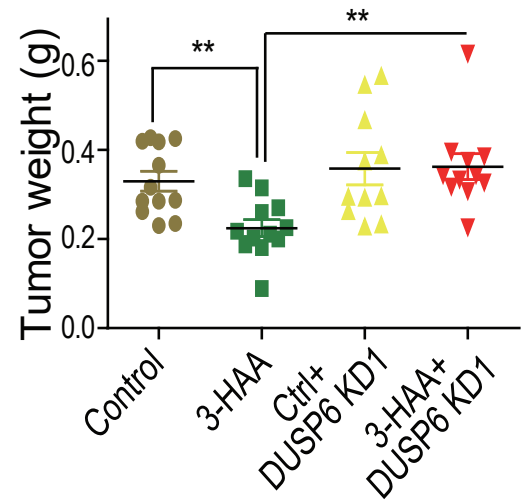

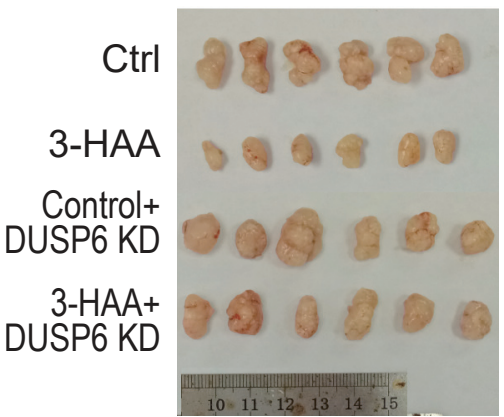


A

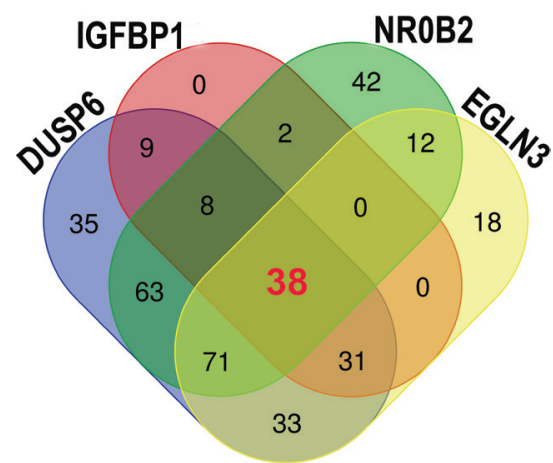

D

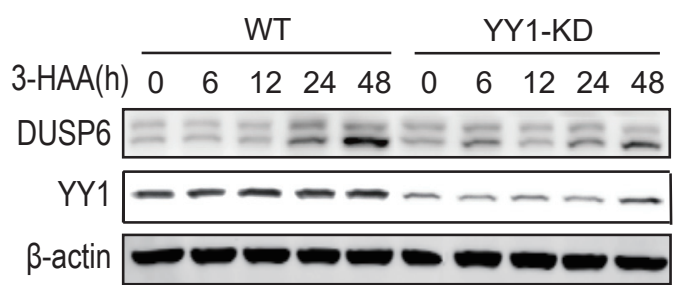

G

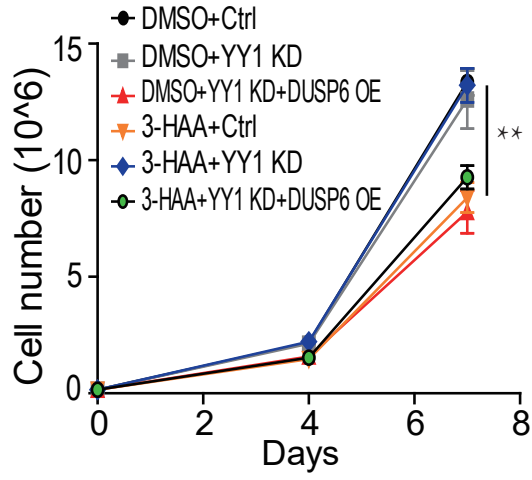

$\mathrm{H}$

AAATTTCCCGGG (mut1: -1598 to -1587) GCCGCCTTTTTT (nonsense sequence)

-1665 DUSP6 promoter $+\frac{1}{\text { luciferase - }}$ pGL4-basic vecto TCCATCCGGCTT (YY1 binding site) CTTCCTTTTTCC (mut2: -1145 to -1134)

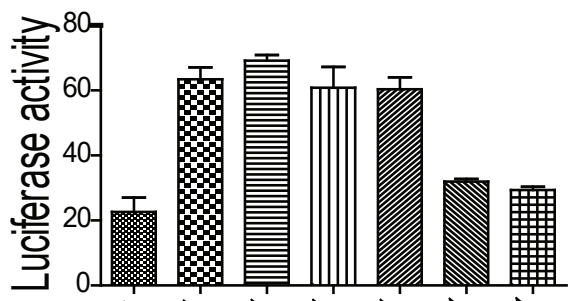
$80,4.45 .450 .45 .45$
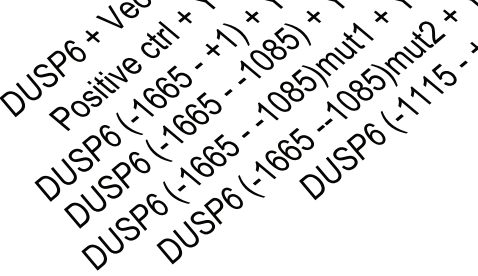

B

3-HAA-regulated TFs on 4 genes chromatin proteins promoter region

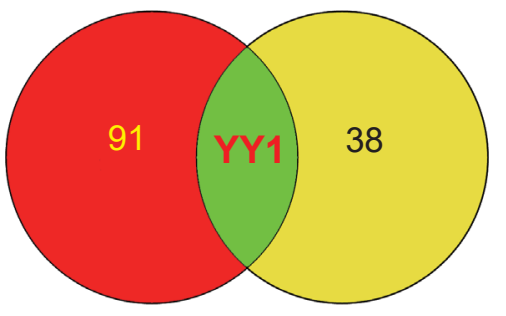

E

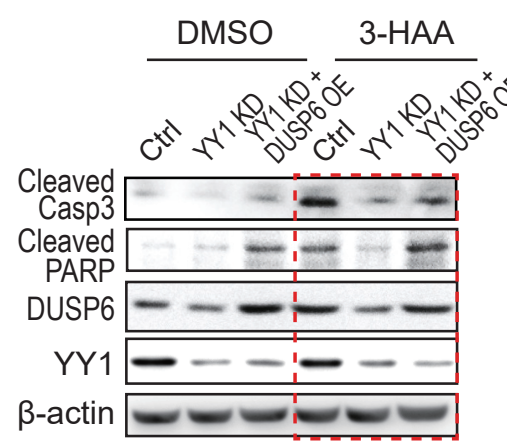

I

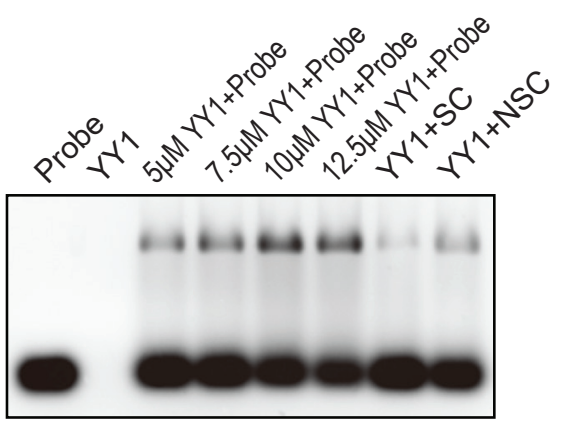

J

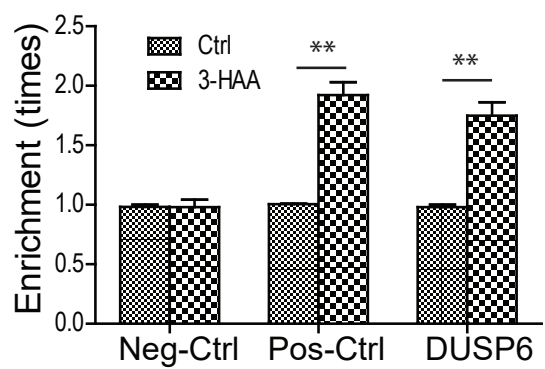

$\mathrm{K}$

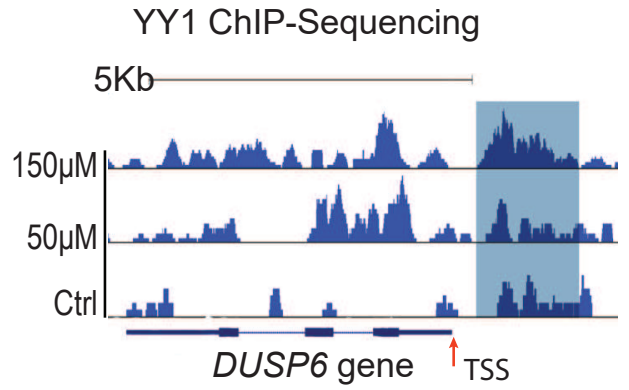

C

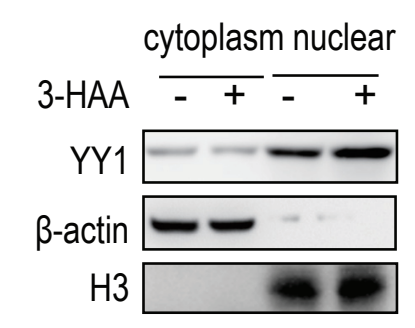

$\mathrm{F}$

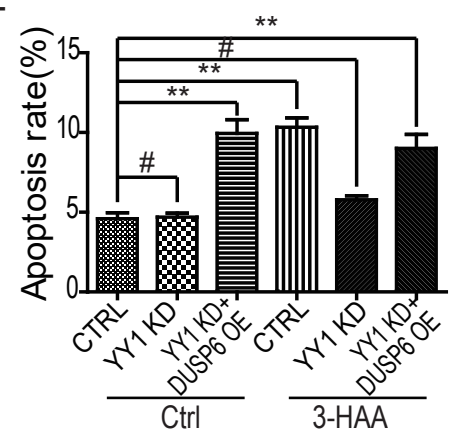

L
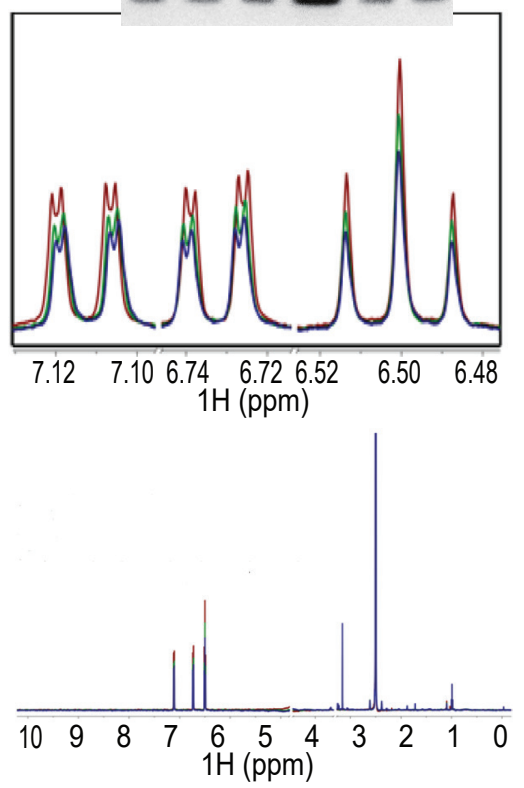

M

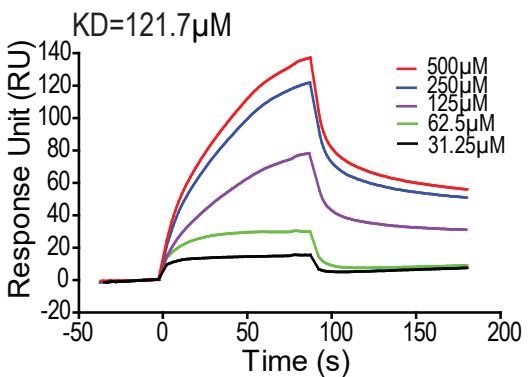


A

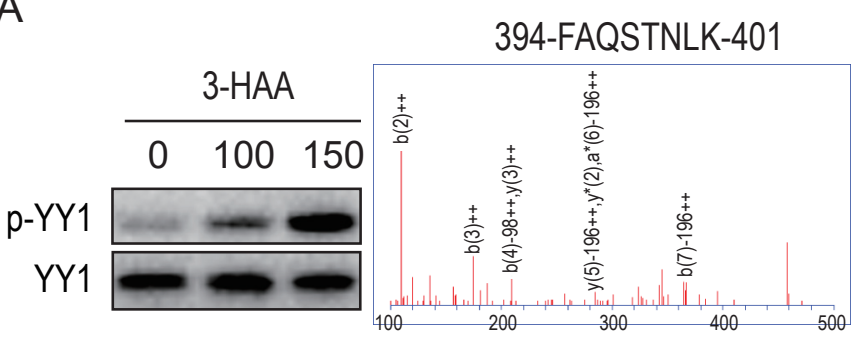

D

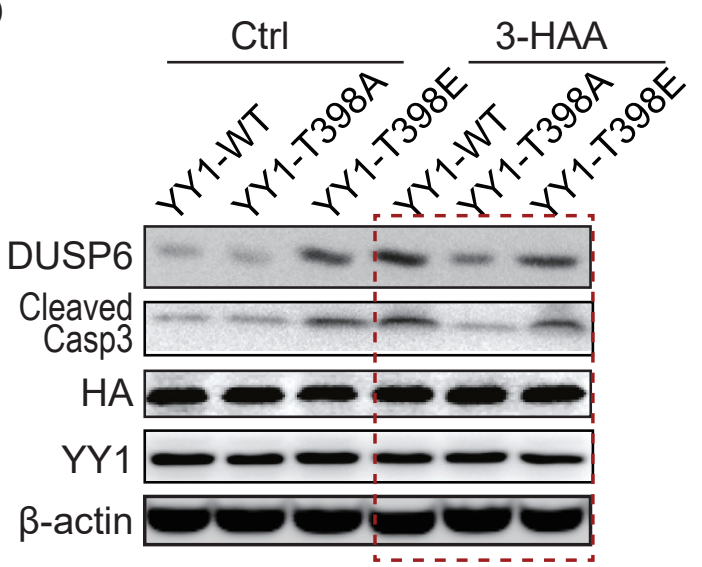

F

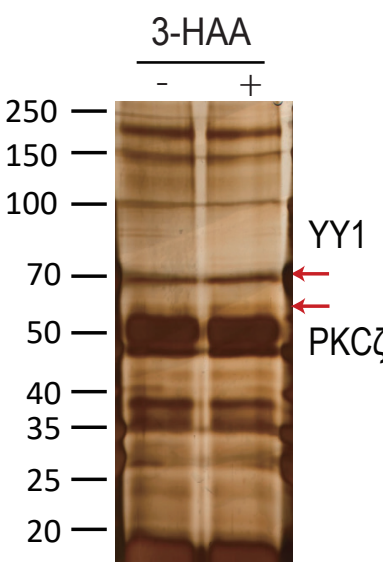

G

J

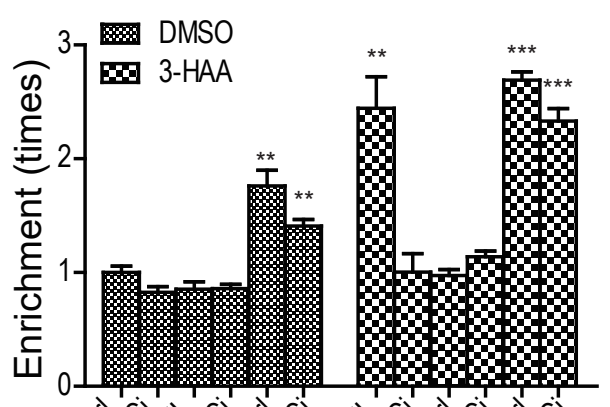

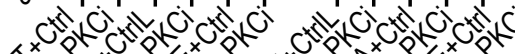

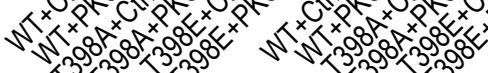

K
B

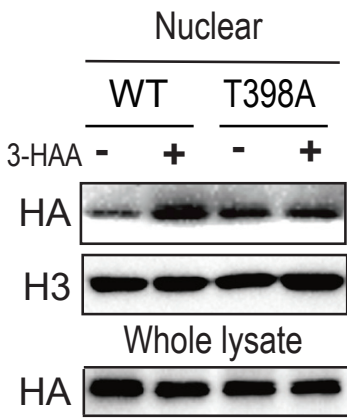

E

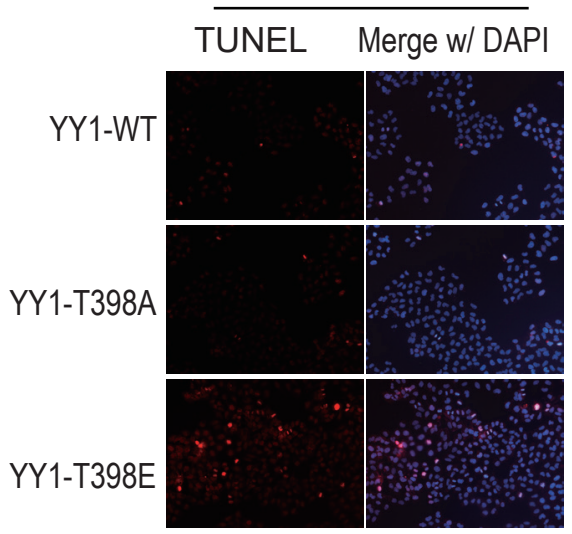

$\mathrm{H}$

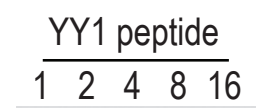

Ctrl

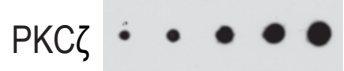

AKT

mTOR

Autoradiogram
C

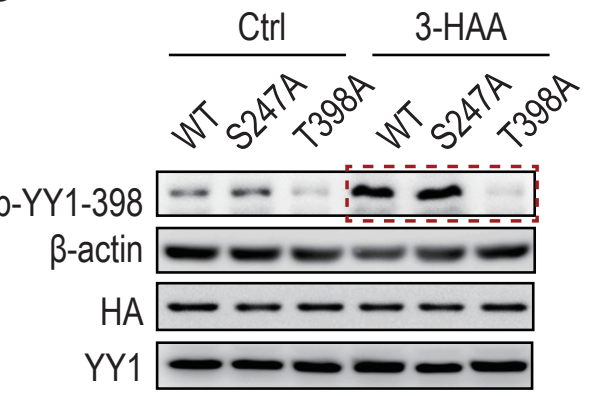

3-HAA

TUNEL Merge w/ DAPI

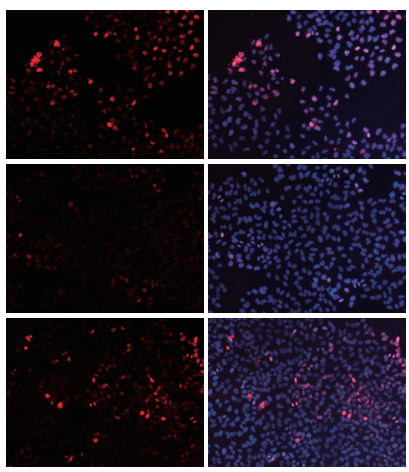

3-HAA

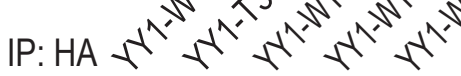

p-YY1-398 — +

$\mathrm{HA}=-\infty-\infty$

YY1 - - - -
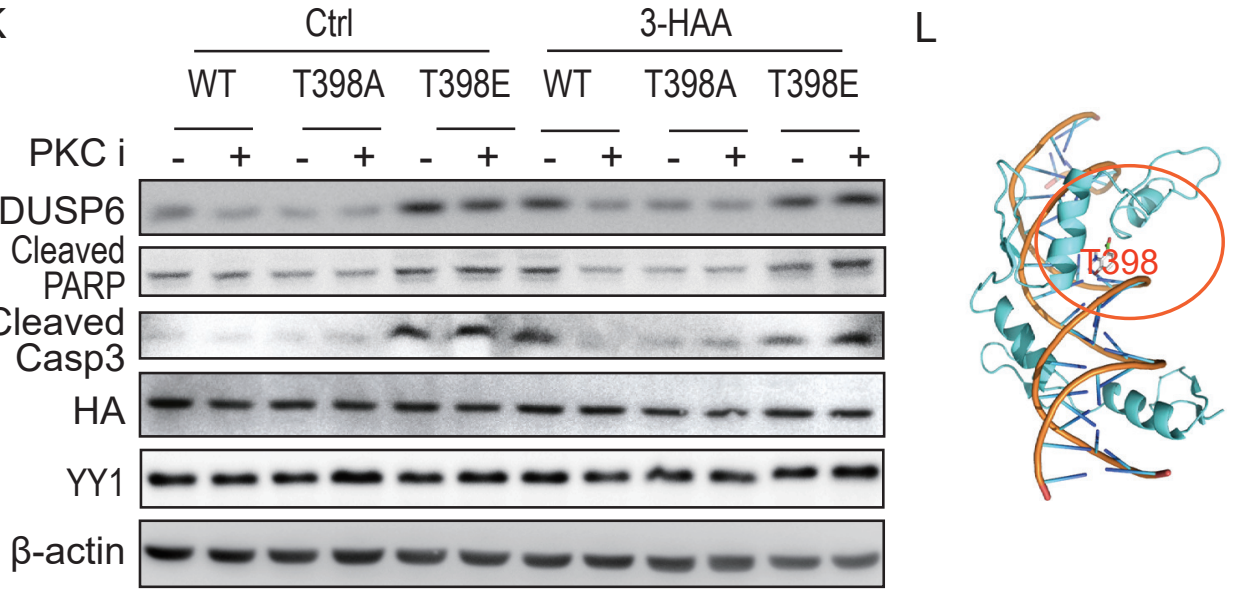
Figure 6

A
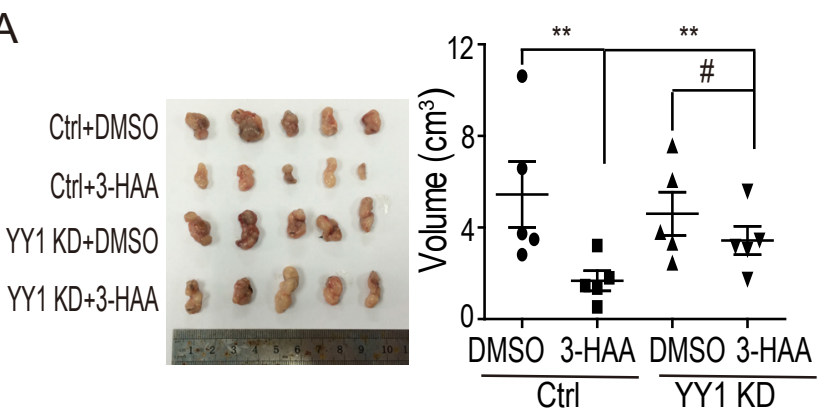

D

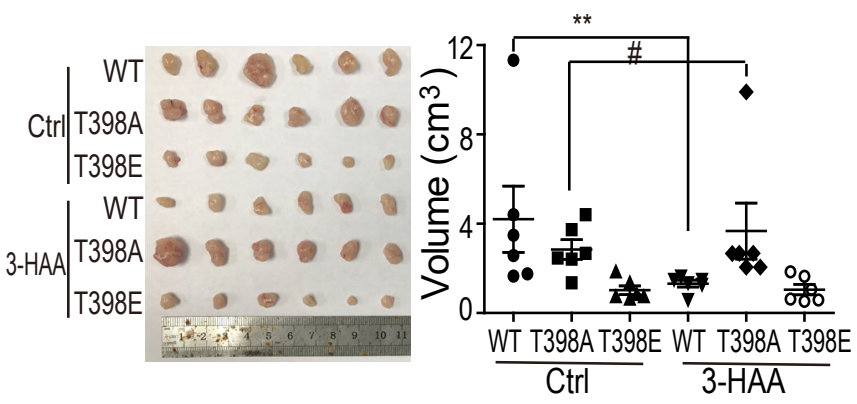

E

C
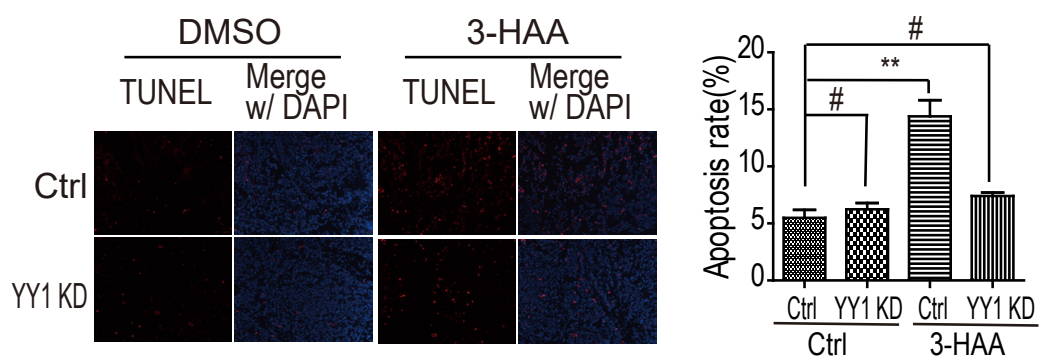

$\frac{\text { DMSO }}{\text { TUNEL } \begin{array}{c}\text { Merge } \\ \text { W/DAPI }\end{array}} \frac{3-\text { HAA }}{\text { TUNEL } \begin{array}{c}\text { Merge } \\ \text { W/DAPI }\end{array}}$
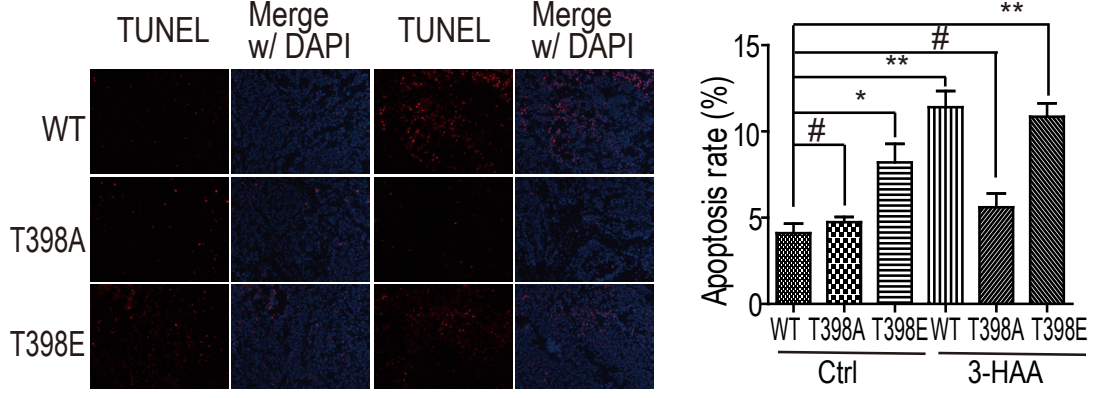

G

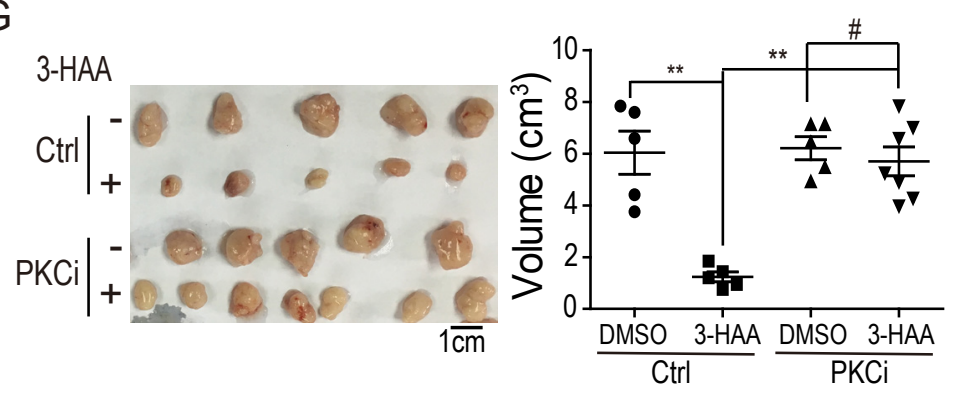

J

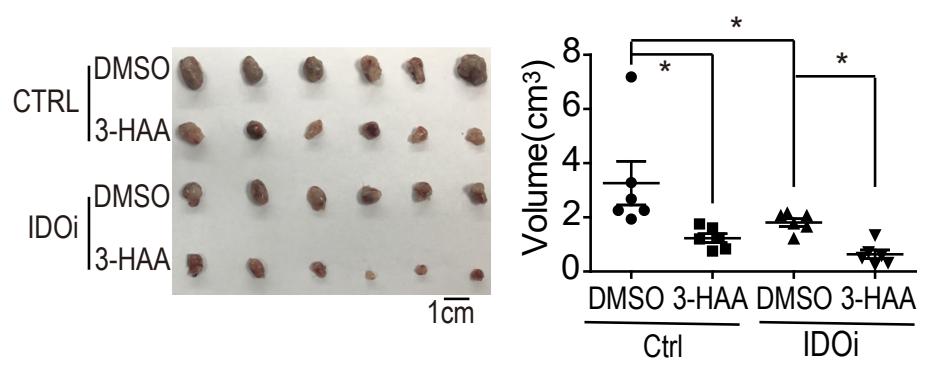

$\mathrm{H}$

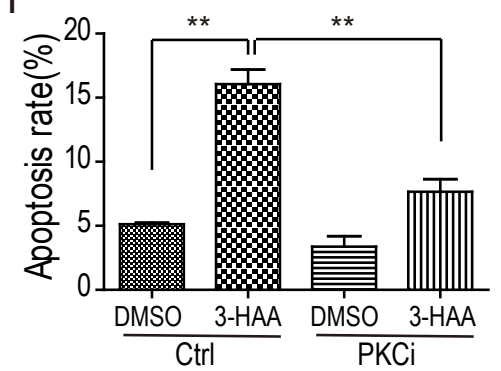

I

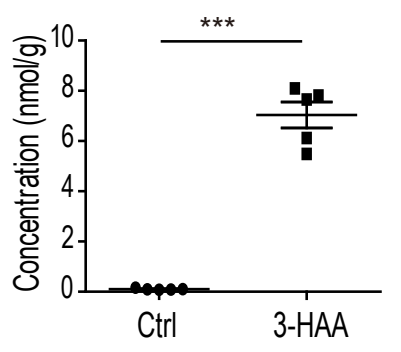

K

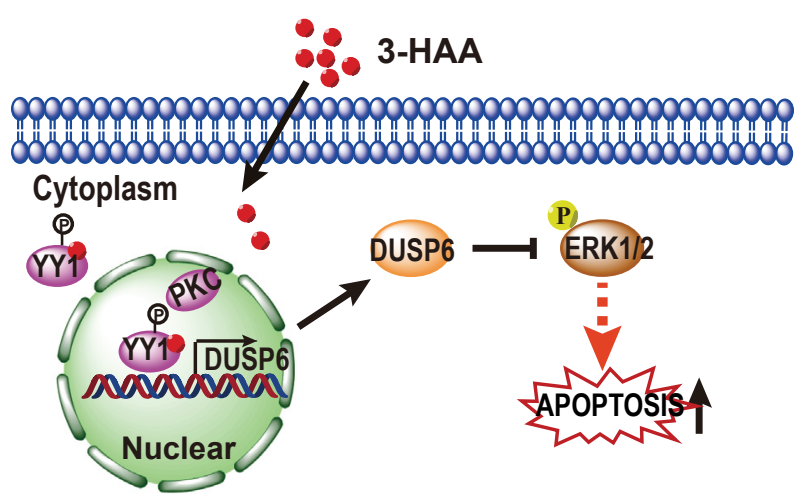


Figure S1 3-HAA is decreased in tumor cells. Related to Figure 1
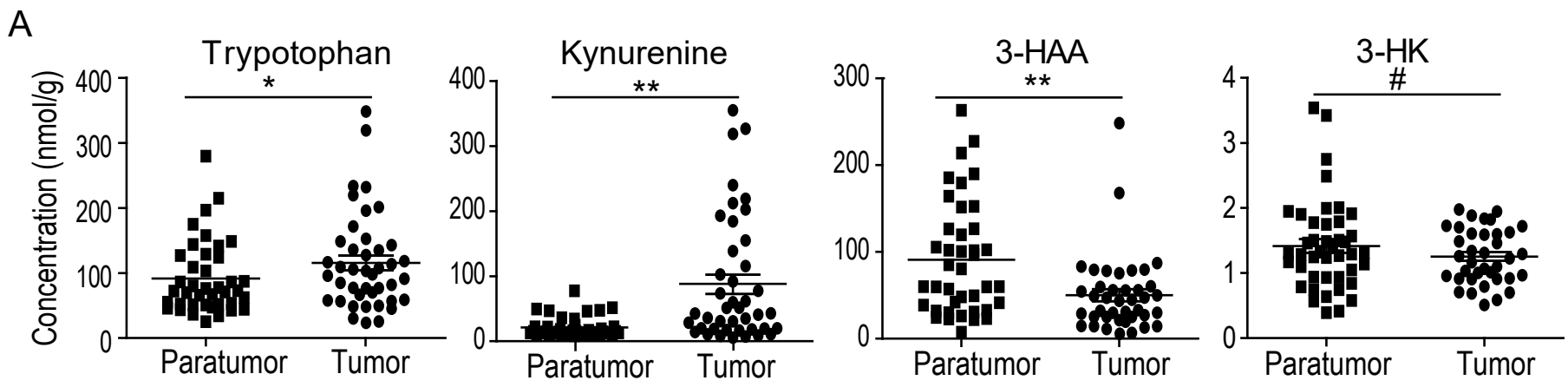

B

KYNU
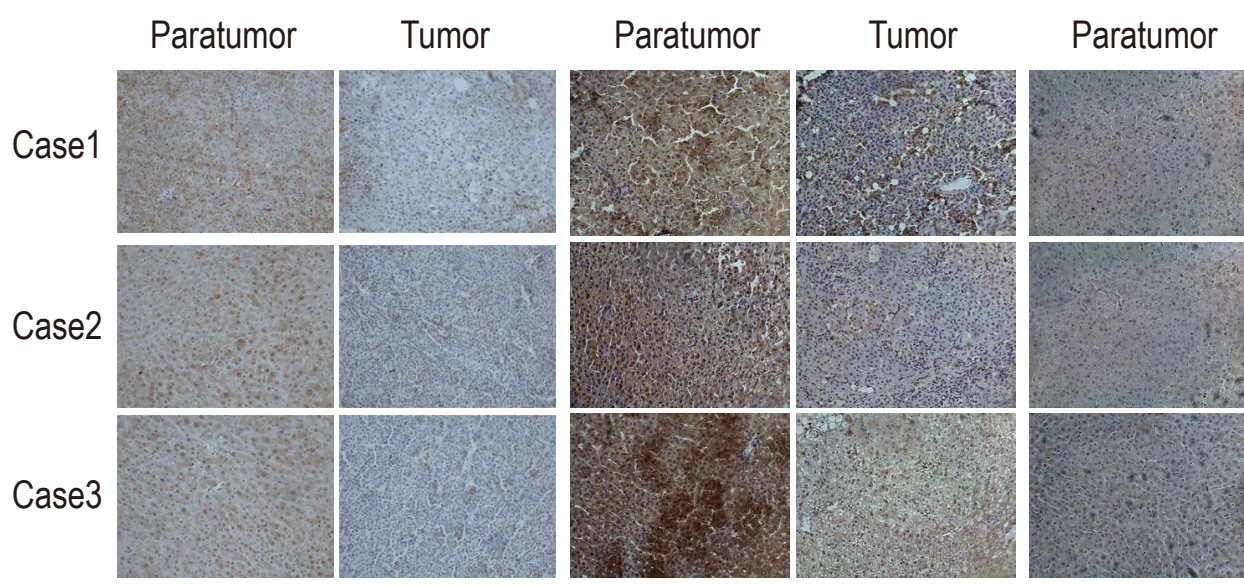

IDO1

HAAO
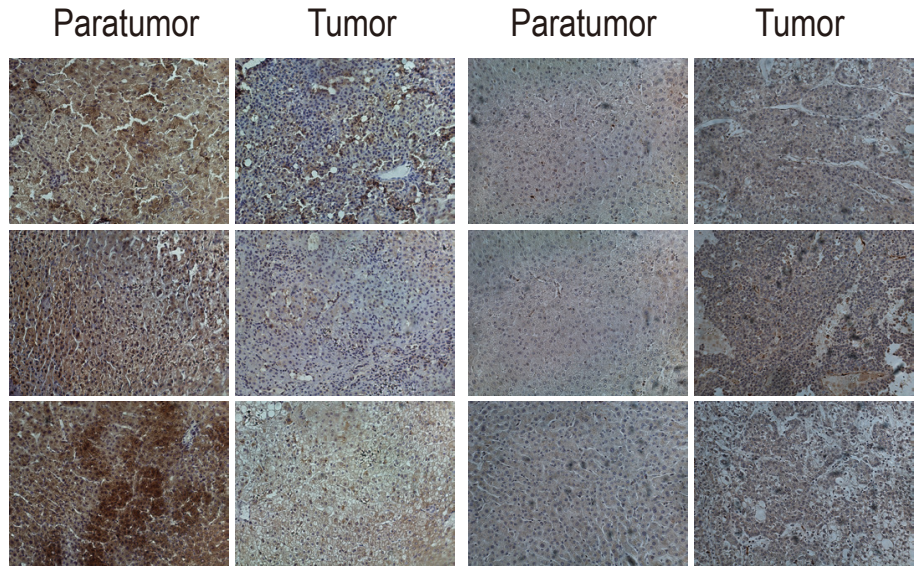

Paratumor Tumor

C
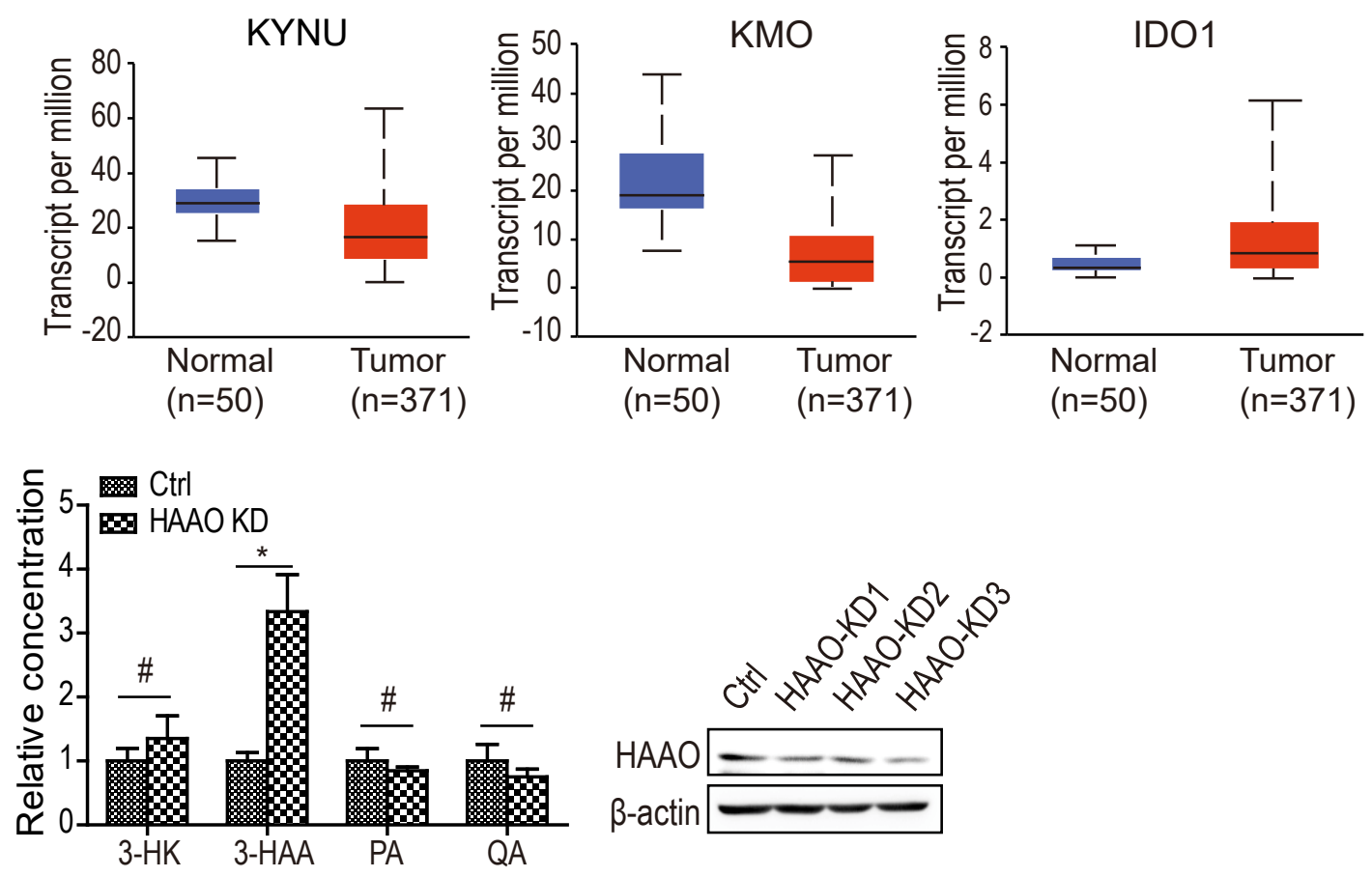

Figure S1 legend

A. The quantitative analysis of general tryptophan catabolites in esophagal carcinoma by mass-spec.

The esophageal carcinoma patients were 42 cases. The ${ }^{*}: p<0.05$; the ${ }^{* *}: p<0.01$; the \#: $p>0.05$.

$B$. The immunohistochemistry staining of KYNU, KMO, IDO and HAAO on HCC samples.

C. The expression profiling of KYNU, KMO, and IDO in the HCC samples by RNA-seq.

D. The quantitative analysis of 3-HK, 3-HAA, PA, and QA in SMMC7721 cells depleted of HAAO. 
Figure S2 3-HAA inhibits tumor growth by inducing apoptosis. Related to Figure2

A

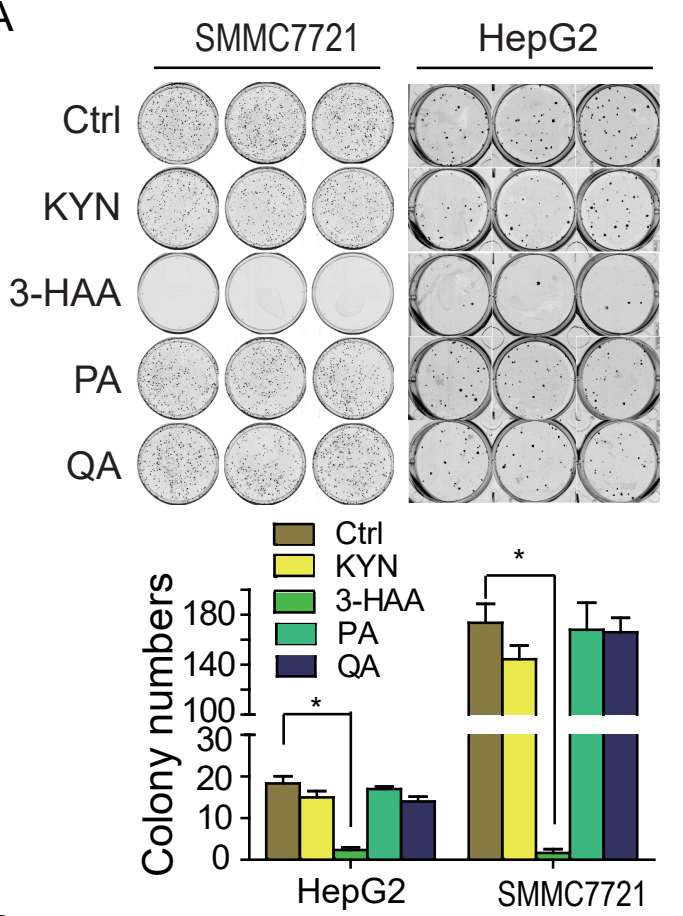

B

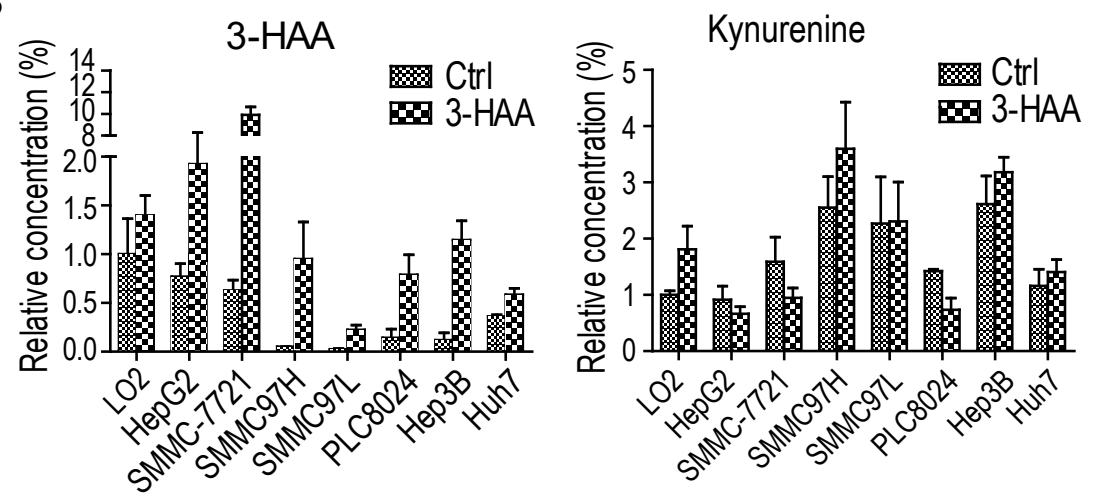

C

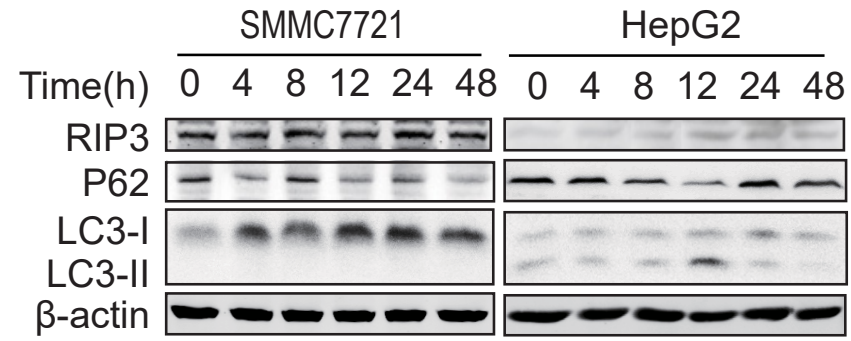

D
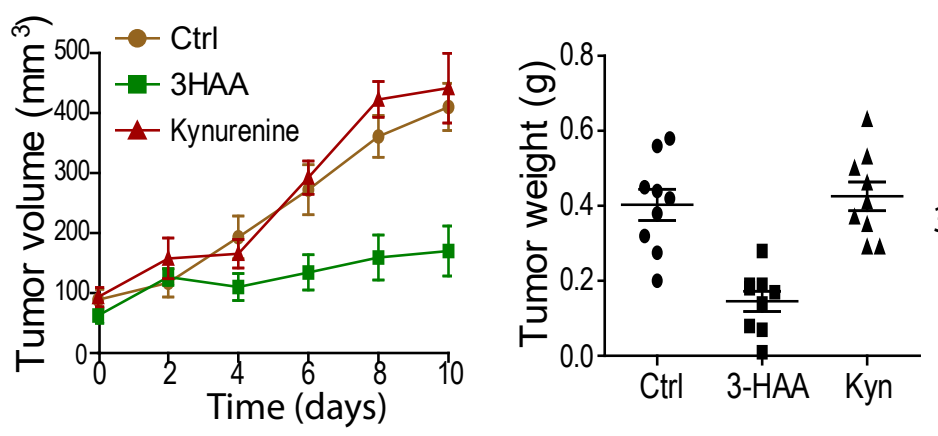

$E$
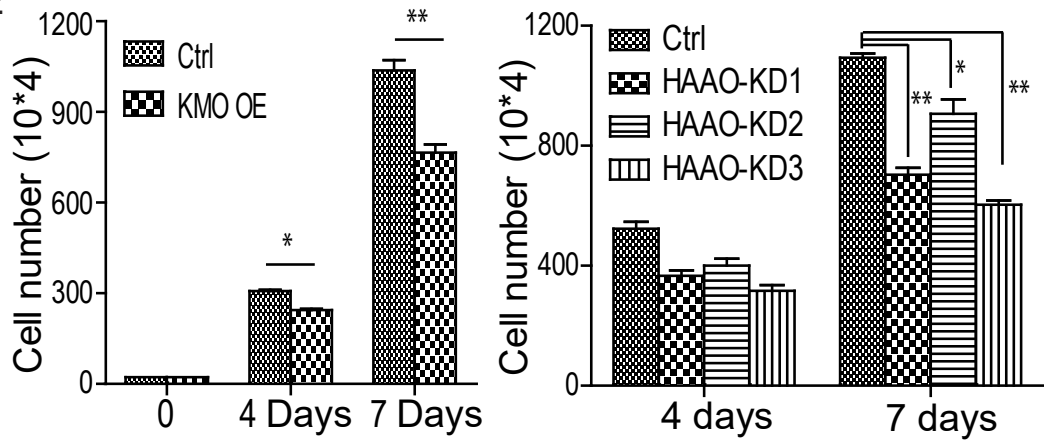

F

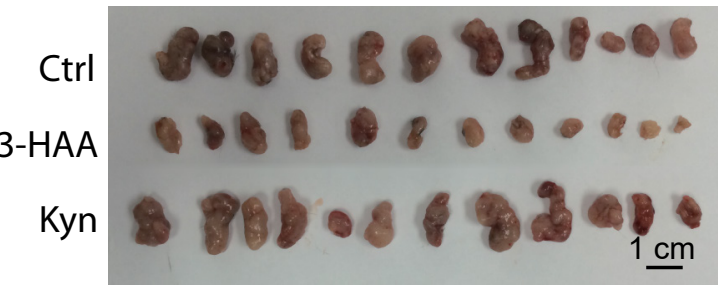

G
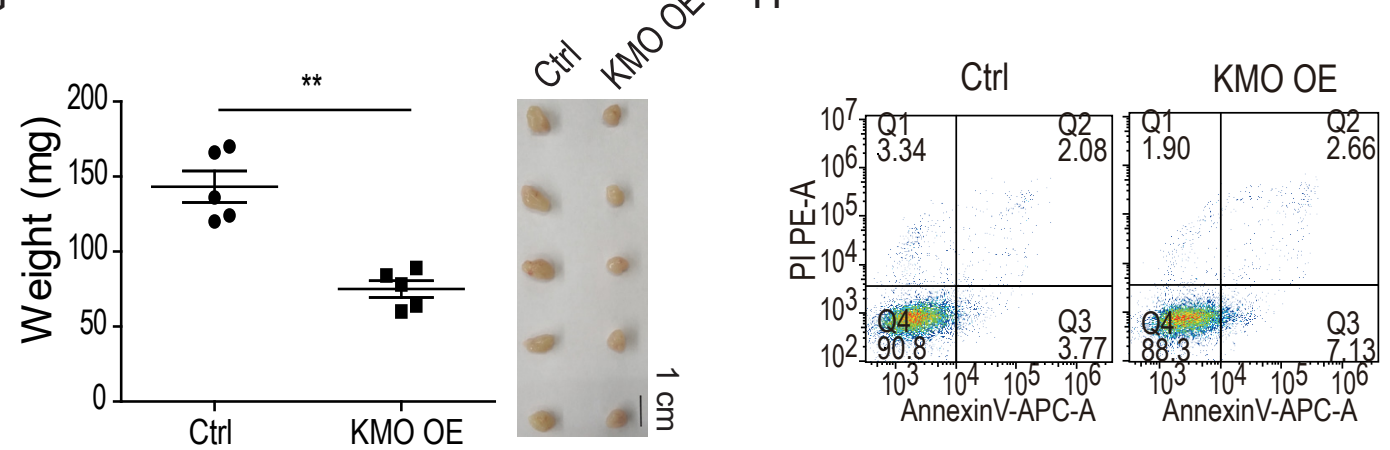
Figure S2

A. The effect of four tryptophan metabolites on colony formation of HCC cells. The concentration of kynurenine, 3-HAA, picolinate and quinolinate was $100 \mu \mathrm{M}$. The cells were treated for 14 days.

B. The concentration of 3-HAA and kynurenine measured by the LC-MS/MS analysis in cells treated with 3-HAA.

C. The cell death marker detection in HCC cells treated with 3-HAA at the dose of $100 \mu \mathrm{M}$.

D. The effects of 3-HAA on the growth of xenograft SMMC7721 tumors. The concentration of both 3-HAA and Kynurenine was $100 \mathrm{mg} / \mathrm{Kg}$.Day.

E. The effect of HAAO and KMO on the cell growth of SMMC7721 cells. The KMO and HAAO was overexpressed or knocked down in SMMC7721 cells, separately.

F. The representative living image of $\mathrm{HCC}$ xenografts expressing KMO.

$\mathrm{G}$. The effect of KMO overexpression on the growth of HCC SMMC7721 xenografts. The cell numbers were $1.5 \times 10^{6}$ for each injection.

$\mathrm{H}$. The flow cytometry analysis of apoptosis. 
Figure S3 3-HAA suppressed tumor growth by upregulating DUSP6 expression. Related to Figure 3

A

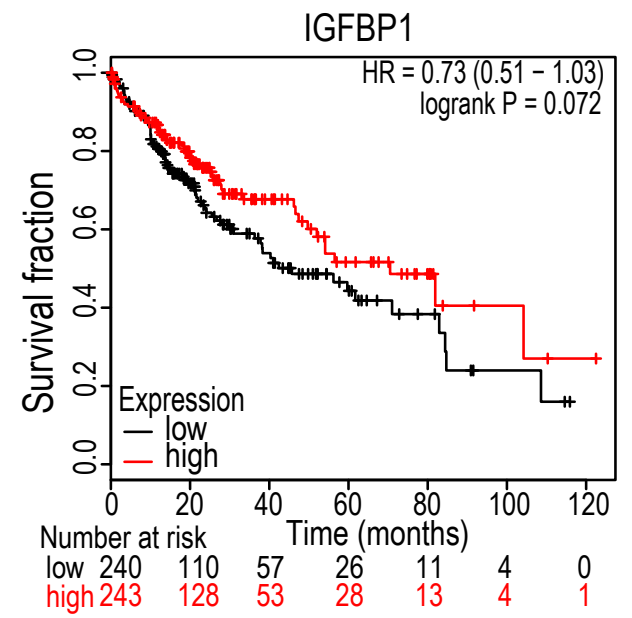

B

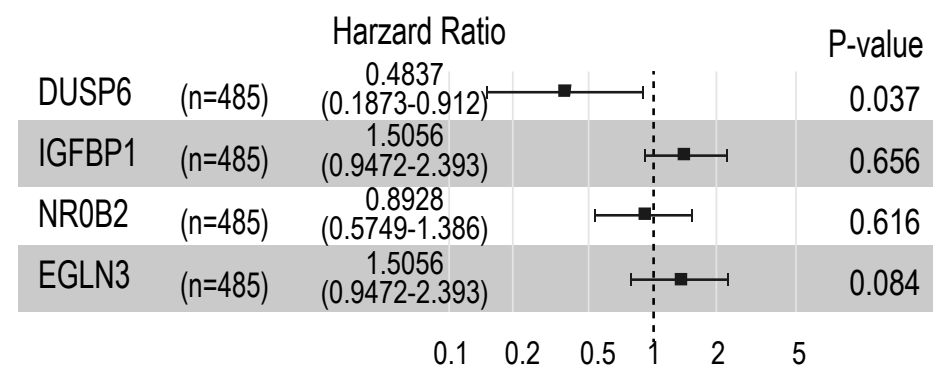

C
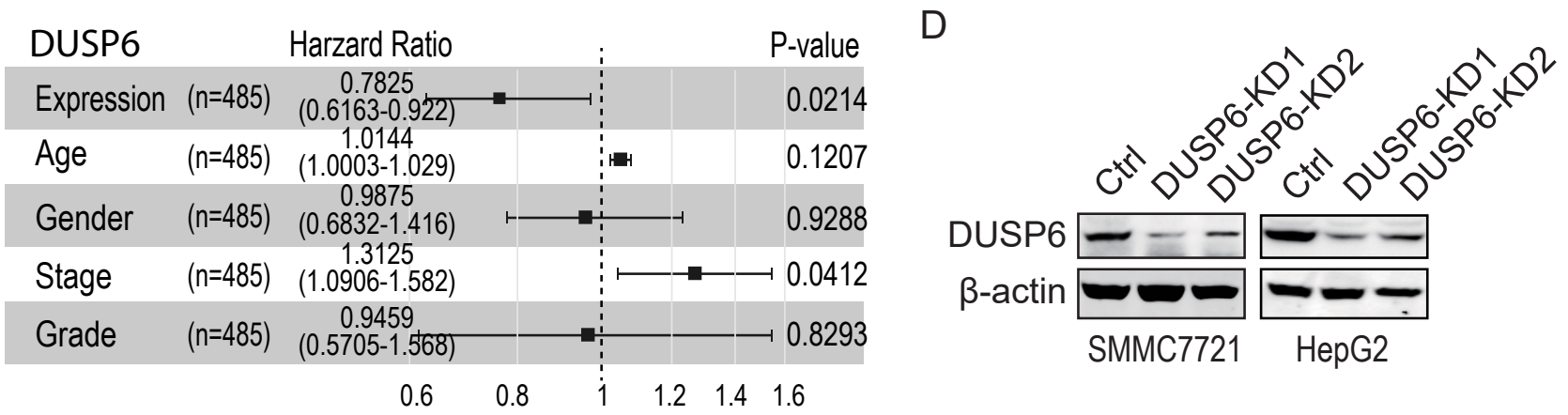

$E$

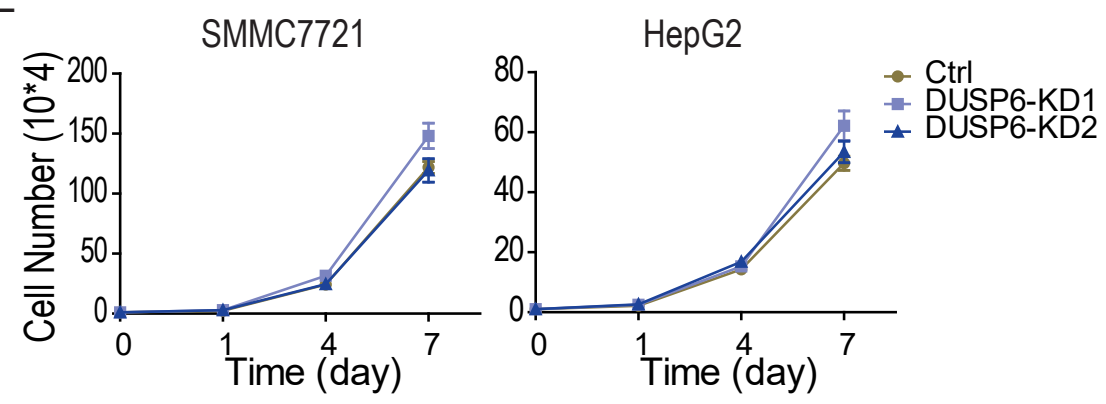

Figure S3 legend

A. The effect of IGFBP1 expression on liver cancer overall survival. The total patient number was 415 , The HCC patients were divided by the median of KMO expression value.

B. The multivariate analysis on the HCC patients' overall survival.

C. Corrective analysis on overall survival of HCC patients with clinical characteristics

D. Knockdown effeciency detection of DUSP6 in SMMC7721 and HepG2 cells.

E. The effects of DUSP6 knockdown on HCC cell growth without 3-HAA. 
Figure S4 3-HAA upregulated DUSP6 expression by binding YY1. Related to Figure 4

A TFs predicted on 4 genes' promoters $\mathrm{C}$

$\begin{array}{lll}\text { SP1 } & \text { KMT2B } & \text { TFAP4 } \\ \text { ELF3 } & \text { KAT7 } & \text { EGR1 } \\ \text { MNT } & \text { MAX } & \text { NFIL3 } \\ \text { POLR2A } & \text { RNF2 } & \text { IKZF5 } \\ \text { YY1 } & \text { MXI1 } & \text { SMAD4 } \\ \text { SP5 } & \text { ZNF580 } & \text { ARID4B } \\ \text { CREM } & \text { ZBTB33 } & \text { THAP11 } \\ \text { KAT8 } & \text { SAP130 } & \text { KLF16 } \\ \text { CREB1 } & \text { GABPB1 } & \text { KDM1A } \\ \text { GATAD2A } & \text { DRAP1 } & \text { ETV5 } \\ \text { IRF2 } & \text { SIN3A } & \text { MXD3 } \\ \text { GTF2F1 } & \text { GATAD1 } & \text { ERF } \\ \text { ZNF792 } & \text { ZGPAT } & \end{array}$

$B$ Increased proteins binding to chromatin $D$ post-3-HAA treatment

$\begin{array}{ll}\text { PGRMC1 } & \text { PRDX6 } \\ \text { CYR61 } & \text { PHB } \\ \text { CIT } & \text { COPB2 } \\ \text { PDCD5 } & \text { LONP1 } \\ \text { TCERG1 } & \text { MDH2 } \\ \text { SEC16A } & \text { HSD17B4 } \\ \text { TRIM24 } & \text { HNRNPM } \\ \text { RNASEH2A } & \text { RARS } \\ \text { ATP5H } & \text { VCP } \\ \text { LUC7L3 } & \text { EIF3B } \\ \text { PGLS } & \text { EEFSEC } \\ \text { ETHE1 } & \text { VBP1 } \\ \text { VTN } & \text { NUTF2 } \\ \text { ALDOA } & \text { GNB1 } \\ \text { ENO1 } & \text { RAC1 } \\ \text { TUBB } & \text { HSPG2 } \\ \text { DLD } & \text { CKAP4 } \\ \text { EZR } & \text { AIMP2 } \\ \text { UCHL3 } & \text { DHRS2 } \\ \text { DSP } & \text { MTA1 } \\ \text { CD44 } & \text { SNX1 } \\ \text { POR } & \text { TRIM29 } \\ \text { ATP5J } & \text { PCM1 } \\ \text { PAICS } & \text { LAMTOR1 } \\ \text { EIF4B } & \text { FAM83H } \\ \text { BRD2 } & \text { PHLDB2 } \\ \text { YY1 } & \text { RHPN2 } \\ \text { FKBP2 } & \text { BAP18 } \\ \text { STOM } & \text { SRRM1 } \\ \text { DCK } & \text { TBC1D15 } \\ \text { TMOD1 } & \text { CACTIN } \\ \text { SSFA2 } & \text { H1FX } \\ & \end{array}$

C

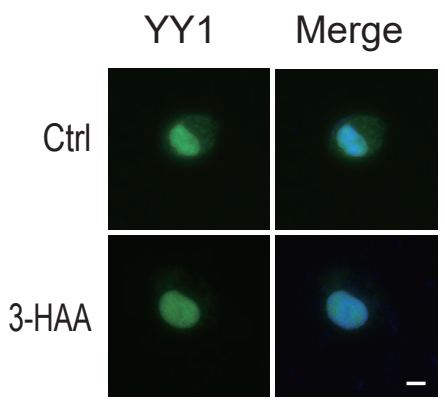

$E$

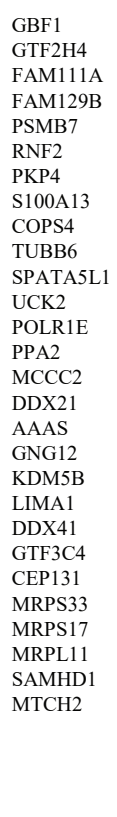

$\mathrm{F}$

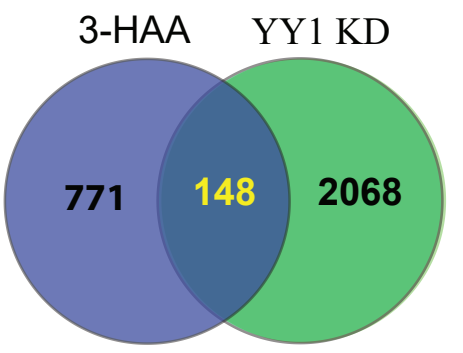

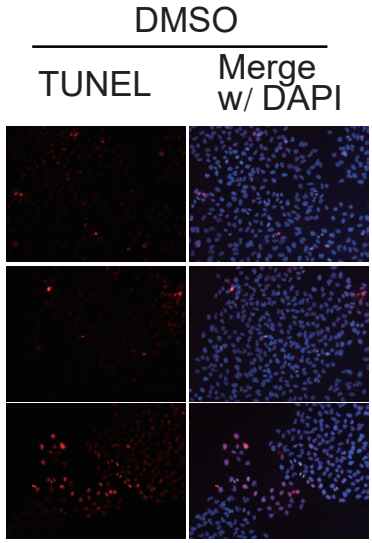

3-HAA $\overline{\text { TUNEL }} \begin{aligned} & \text { Merge } \\ & \text { W/ DAPI }\end{aligned}$

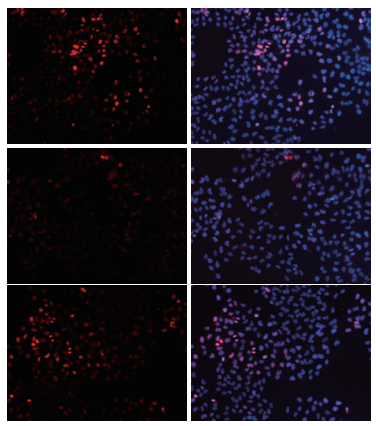

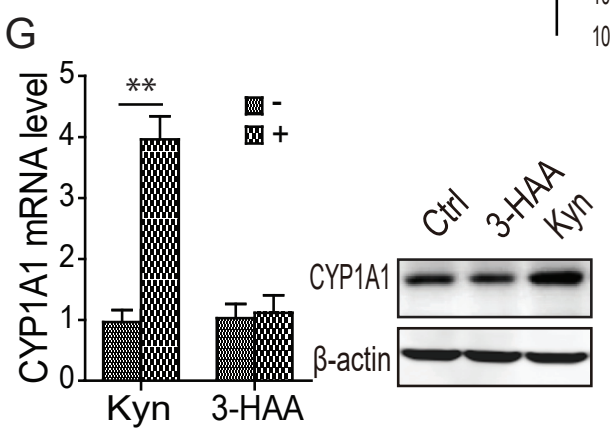
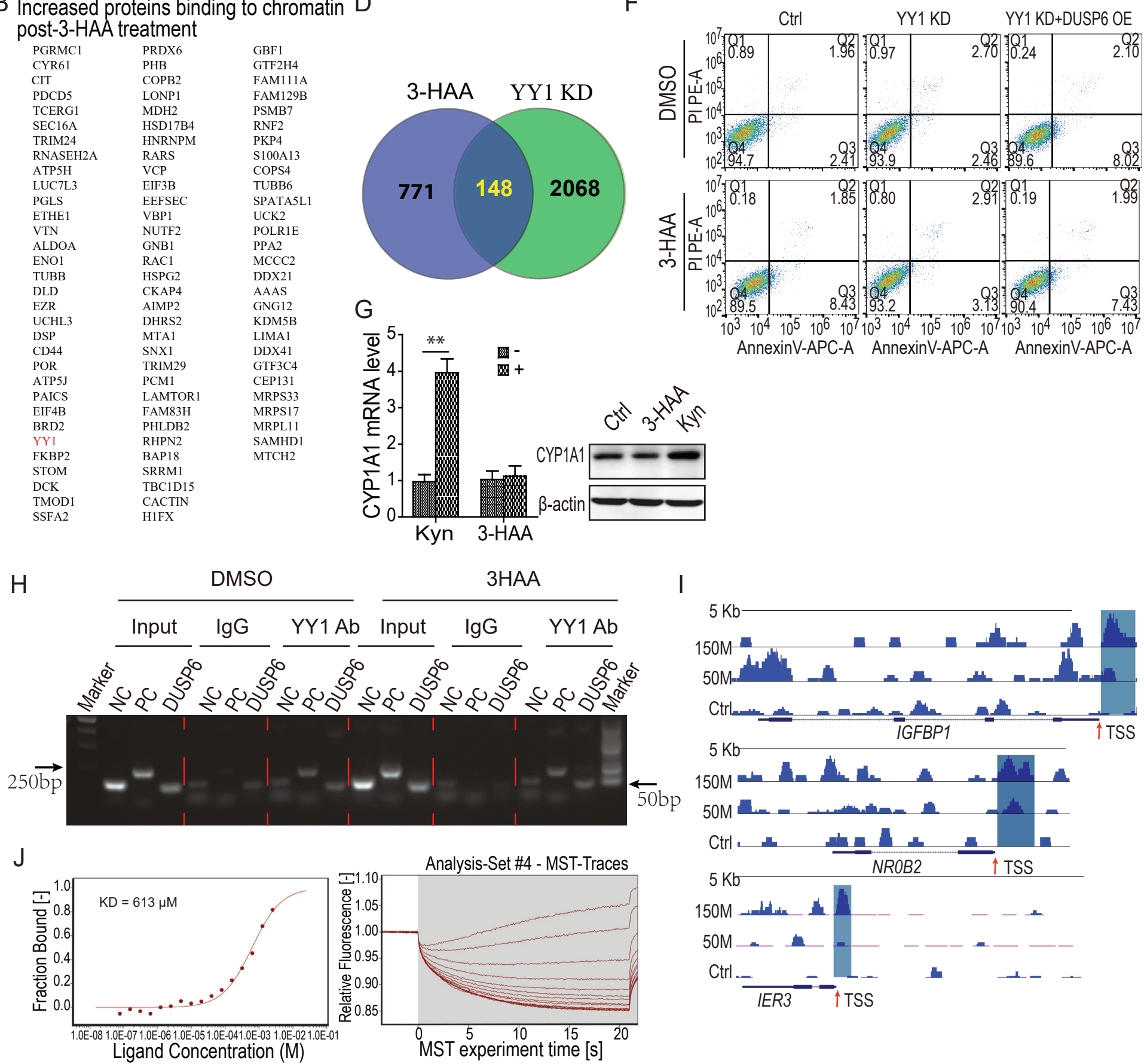

Ligand Concentration (M)

MST experiment time $[\mathrm{s}]$ 
Figure S4

A. The common transcription factors potentially binding to the promoter region $(-5000$ to +1$)$ of top 4 genes, which was up-regulated in 3-HAA-treated SMMC7721 cells.

B. Proteins increasely binding to chromatin after 3-HAA treatment, analyzed by quantitative proteomics analysis.

C. The localization of YY1 with or without 3-HAA treatment.

D. The common genes regualted by both 3-HAA and YY1.

E. The apoptosis analysis by TUNEL assay in SMMC7721 cells depleted of YY1 and/or overexpressing DUSP6.

F. The apoptosis analysis by flow cytometry in SMMC7721 cells depleted of YY1 and/or overexpressing DUSP6.

G. 3-HAA dose not upregulate CYP1A1 as kynurenine does. The dose for borh Kyn and 3-HAA was $100 \mu \mathrm{M}$.

$\mathrm{H}$. The ChIP-PCR analysis. The positive control (PC) is TP53.

I. The peak of ChIP-Seq on the promoter of 3 representative upregulated genes.

J. The dissociation constant of 3-HAA to YY1 determined by MicroScale Thermophoresis analysis (MST). 
Figure S5 PKC $\zeta$ phosphorylates YY1 at Thr398 in response to 3-HAA. Related to Figure 5

A
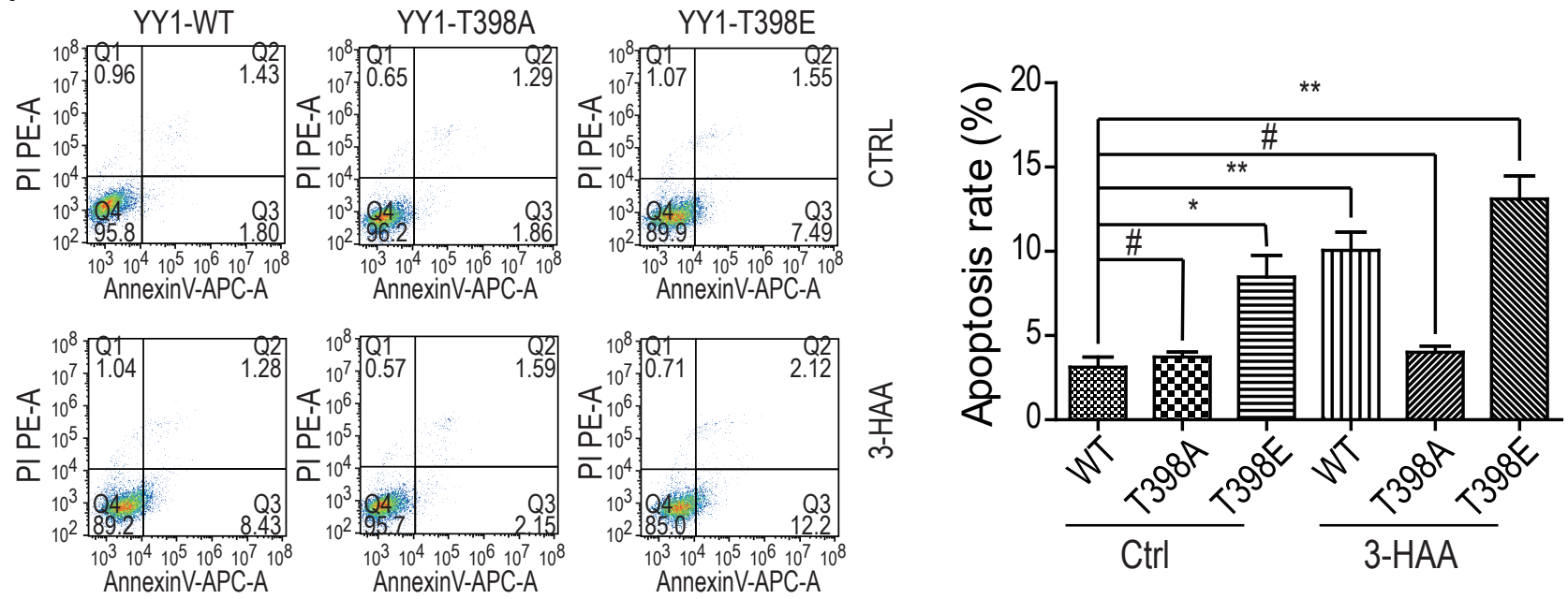

B

C

$\begin{array}{lcc}\text { YY1 binding protein } & \text { Control } & \text { 3-HAA } \\ \text { PPIB } & \checkmark & \checkmark \\ \text { FLG2 } & \checkmark & \\ \text { LYZ } & \checkmark & \checkmark \\ \text { HSPA5 } & \checkmark & \checkmark \\ \text { HSPA9 } & \checkmark & \checkmark \\ \text { HSPD1 } & \checkmark & \checkmark \\ \text { Exportin 1 } & \checkmark & \\ \text { HRNR } & & \checkmark \\ \text { HNRNPA2B1 } & & \checkmark \\ \text { SLC25A4 } & & \checkmark \\ \text { PDIA6 } & & \checkmark \\ \text { HNRNPA0 } & & \checkmark \\ \text { RuvBL1 } & \checkmark & \checkmark \\ \text { RuvBL2 } & \checkmark & \checkmark \\ \text { PKC } & & \checkmark\end{array}$

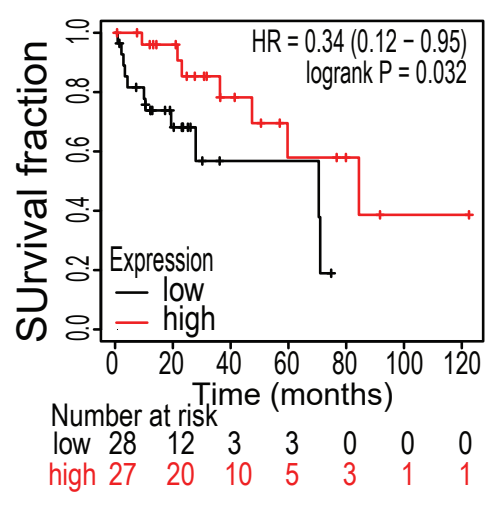

Figure S5 legend

A. The apoptosis of SMMC7721 cells determined by the flow cytometry analysis. The concentration of 3-HAA was $100 \mu \mathrm{M}$.

B. The representative YY1 binding proteins identified by protein mass-spect analysis following coimmunoprecipitation.

C. The effect of $\mathrm{PKC} \zeta$ expression on grade 1 liver cancer overall survival. The total patient number was $55, p<0.05$. 
Figure S6 The T398 phosphorylation of YY1 is critical for 3-HAA-suppressed HCC xenografts growth. Related to Figure 6

A
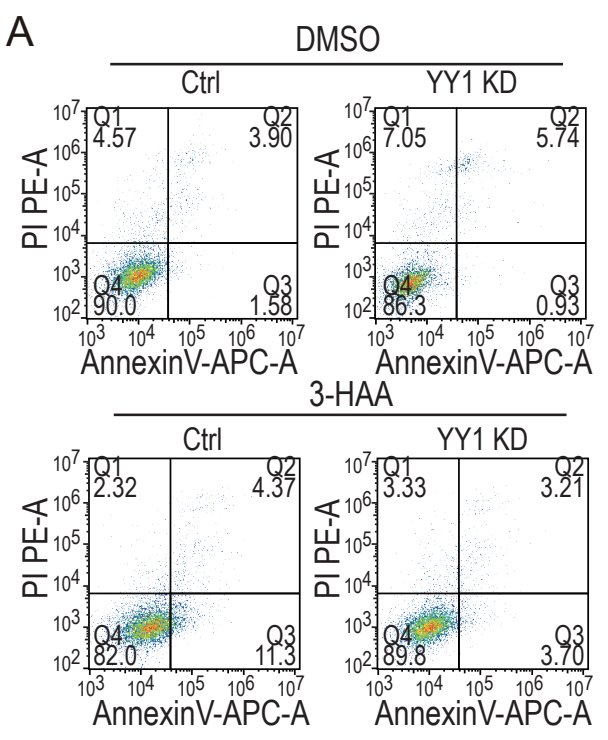

$\mathrm{D}$

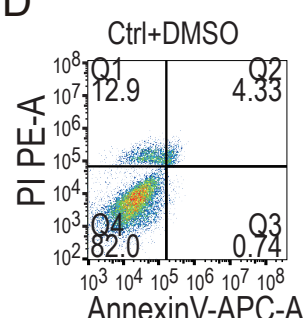

PKCi+DMSO

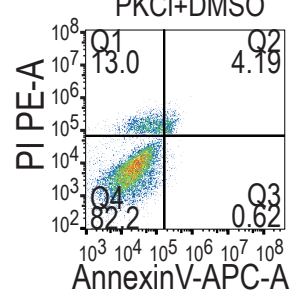

AnnexinV-APC-A

PKCi+3-HAA

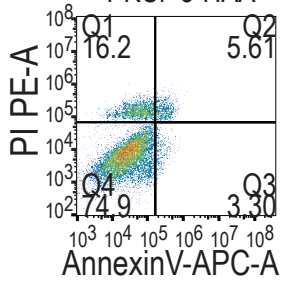

B
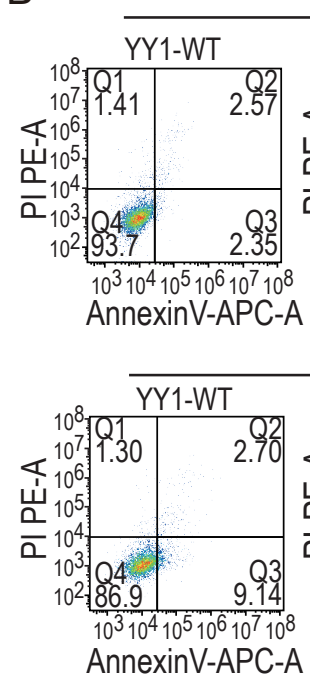

E
DMSO

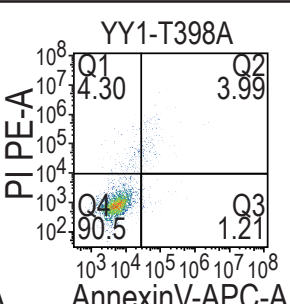

3-HAA

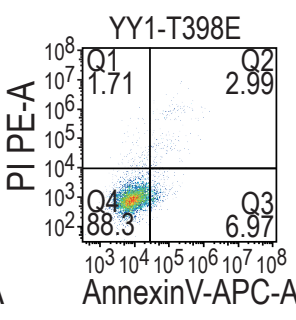

C
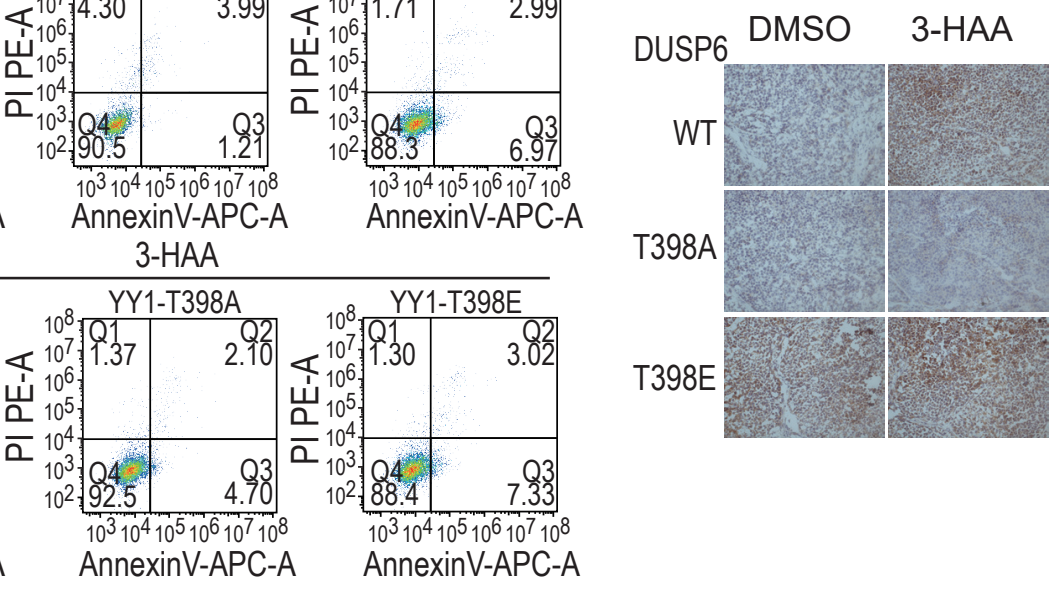

$\mathrm{F}$

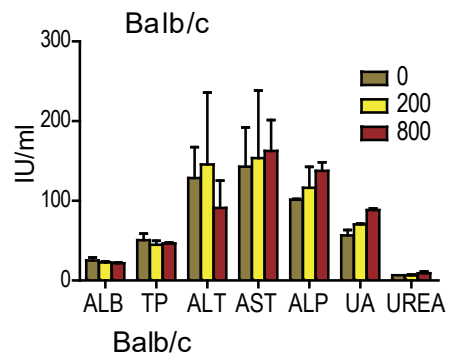

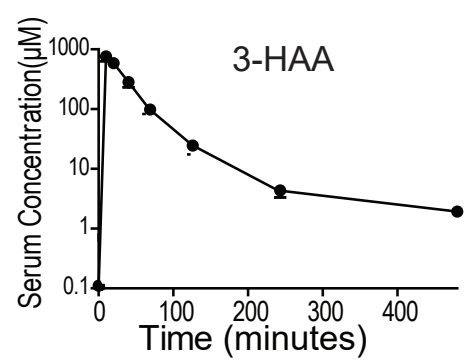

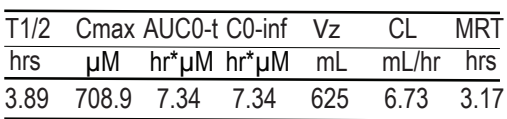

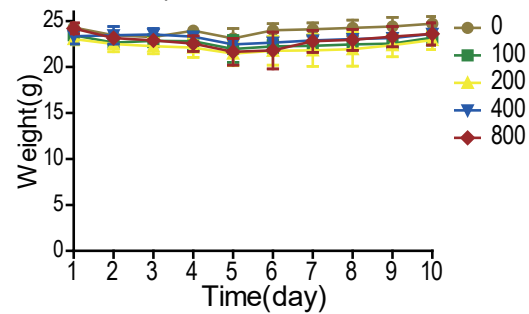

Figure S6 legend:

A. The apoptosis analysis of tumors by flow cytometry. The concentration of 3-HAA was $100 \mathrm{mg} / \mathrm{kg}$.day.

B. The apoptosis analysis of tumors by flow cytometry. The concentration of 3-HAA was $100 \mathrm{mg} / \mathrm{kg}$.day.

C. The DUSP6 expression analysis by IHC in xenografts.

D. The apoptosis analysis of tumors by flow cytometry. The concentration of PKCi was $0.8 \mathrm{mg} / \mathrm{kg}$.day.

E. Plasma concentration versus time in C57 mice following an intraperitoneal injection of $100 \mathrm{mg} / \mathrm{kg}$ of 3-HAA (mean \pm S.D.). Following a single Intraperitoneal dose, the mean plasma T1/2 of 3-HAA were $3.89 \mathrm{hrs}$ and $4.13 \mathrm{hrs}$, respectively. T1/2, elimination half-life; Cmax, maximum concentration; AUC0-t, area under the curve of a plasma concentration from time zero to time $t$; AUC0-inf, area under the plasma concentration-time curve from time zero to infinity; Vz, volume of distribution; CL, total plasma clearance of drug; MRT, mean residence time.

F. The effect of 3-HAA on the liver/renal function and body wight of Balb/c mice. 


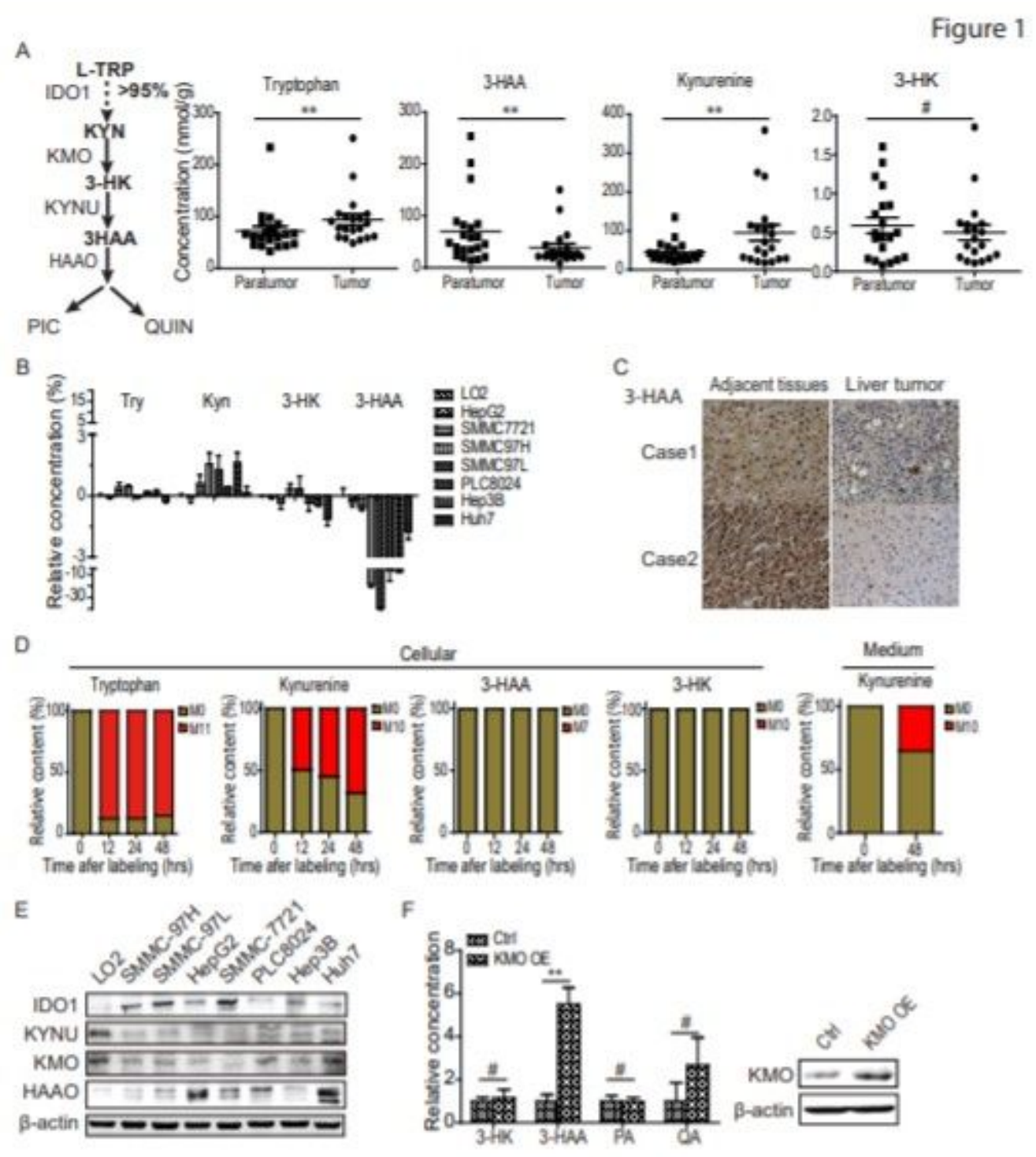

\section{Figure 1}

3-hydroxyanthranilic acid (3-HAA) is selectively decreased in hepatocellular carcinoma A. Quantitative analysis of tryptophan metabolites by LC-MS/MS and GC-MS in HCC and corresponding paratumor tissues. B. Quantitative analysis of tryptophan metabolites by LC-MS/MS and GC-MS in normal hepatic cells and HCC cells. C. Immunohistochemistry staining of 3-HAA in clinical HCC samples. D. Metabolic flux analysis of tryptophan metabolites in Huh7 and HepG2 cells. L-Tryptophan was completely 13Clabelled. The content of tryptophan catabolites in cells and medium was assessed using LC-MS/MS. The M0 stands for no carbon in tryptophan was 13C-labelled, M11 stands for all 11 carbon in tryptophan were 13C-labelled. E. Expression analysis of metabolic enzymes involved in 3-HAA generation. F. The effect of 
KMO overexpression on the concentration of endogenous 3-HAA and tryptophan catabolites in SMMC7721 cells.

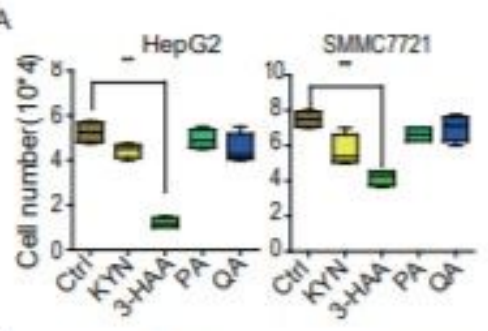

c

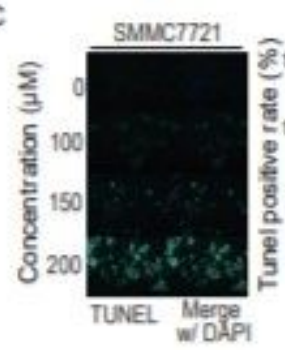

E

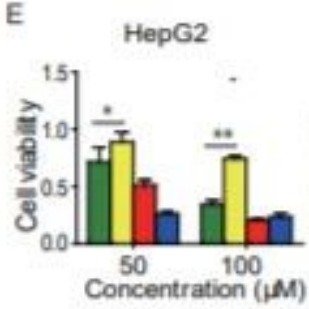

G
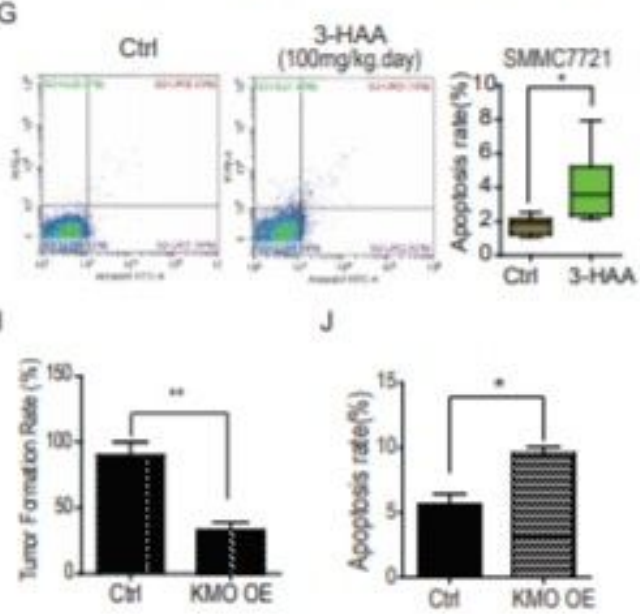

B

D

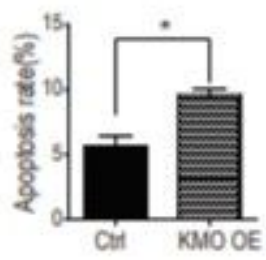

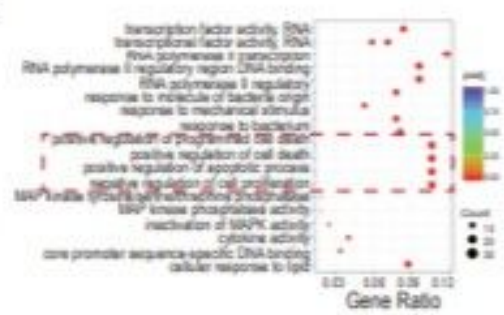

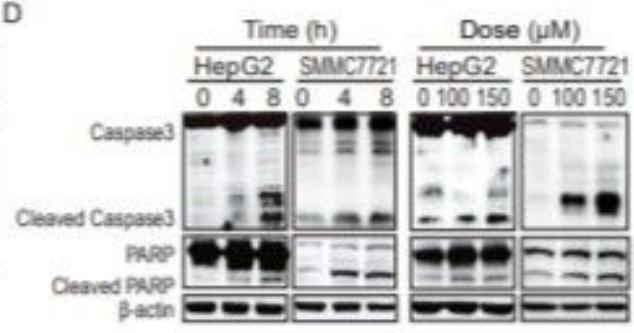

$\mathbf{F}$

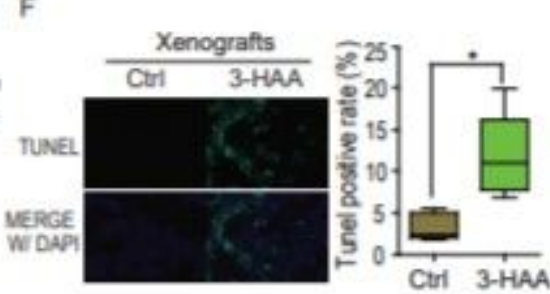

H
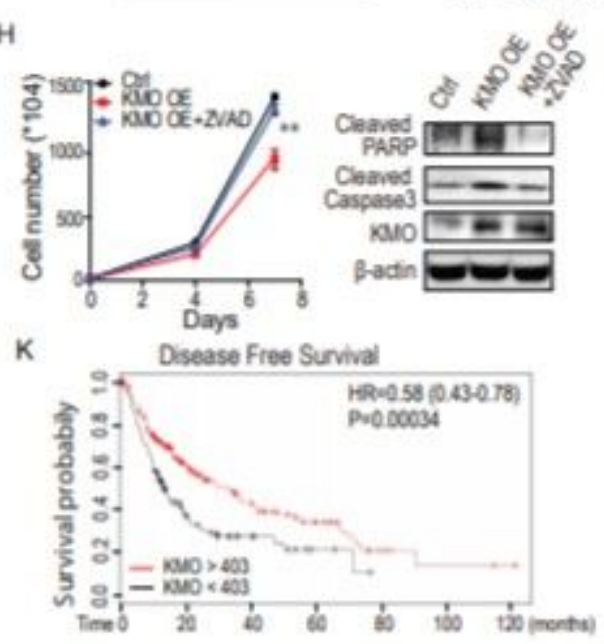

K

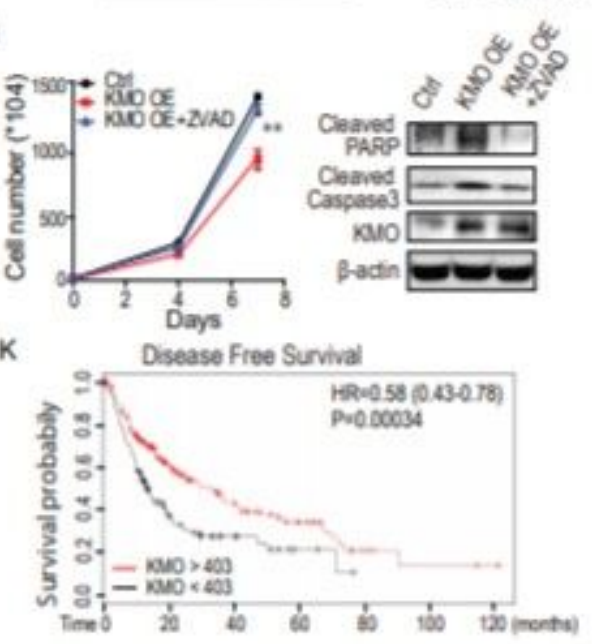

Figure 2

\section{Figure 2}

3-hydroxyanthranilic acid (3-HAA) induces apoptosis of hepatocellular carcinoma cells A. Analysis of the growth of HCC cells treated for 4 days with one of four tryptophan metabolites $(100 \mu \mathrm{M})$. B. Gene Ontology (GO) enrichment analysis of HCC SMMC7721 cells treated by 3-HAA for 8 hours. C. Apoptosis analysis by TUNEL assay in SMMC7721 cells treated for $24 \mathrm{~h}$ with $3-\mathrm{HAA}$ at the indicated dose. D. Analysis of apoptosis signaling in HCC cells treated with $100 \mu \mathrm{M} 3-\mathrm{HAA}$ for indicated time or treated for $12 \mathrm{~h}$ with 3-HAA at the indicated dose. E. The effects of three types of inhibitors on 3-HAA-induced HCC 
cell death. ZVAD: apoptosis inhibitor $(20 \mu \mathrm{M})$; Nec1: necrosis inhibitor $(100 \mu \mathrm{M})$; 3-MA: autophagy inhibitor ( $5 \mathrm{mM})$. The dose of 3-HAA was $100 \mu \mathrm{M}$. Cells were treated for 4 days. F and G. Apoptosis analysis by TUNEL assay and flow cytometry in SMMC7721 xenografts with and without 3-HAA treatment. The dose of 3-HAA was $100 \mathrm{mg} / \mathrm{kg} \cdot$ day. Mice were treated for 7 days and sacrificed on day 10 . $\mathrm{H}$. The influence of endogenous 3-HAA on cell growth and apoptosis signaling of HCC cells. The KMO was overexpressed in SMMC 7721. The **: $p<0.01$. The concentration of 3-HAA and ZVAD was $100 \mu M$ and $20 \mu \mathrm{M}$, respectively. I. Tumor formation analysis of HCC cells overexpressing KMO. The effect of endogenous 3-HAA on xenografted tumor formation. J. The effect of KMO overexpression on apoptosis of SMMC7721 cells. K. The effect of KMO expression on the disease-free survival of HCC patients. The patients were divided by the median of KMO expression value.

A
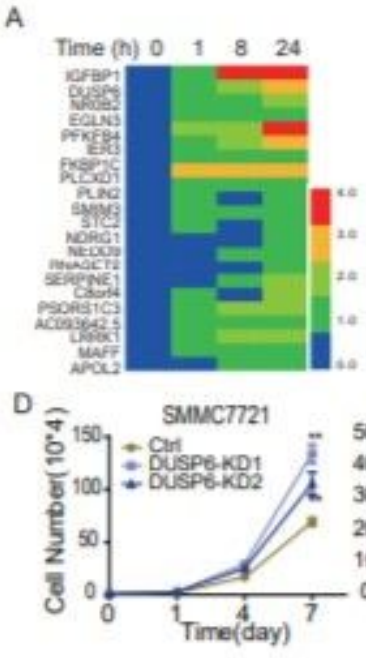

E

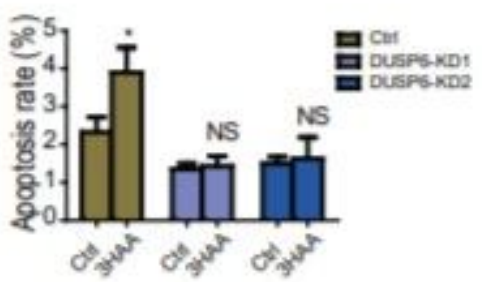

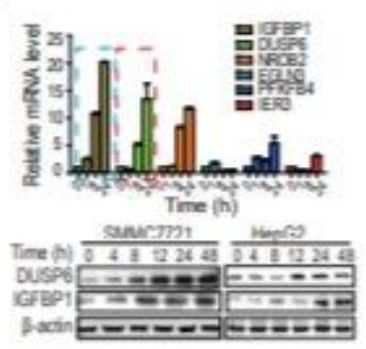

F

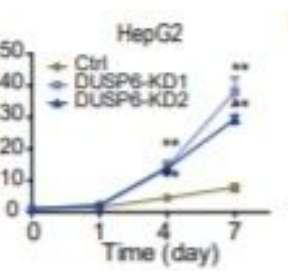

p-ERK1/2

ERK1/2

$\mathrm{BAD}$

BCL2

$\mathrm{BCL}-\mathrm{XL}$

Casp3

C

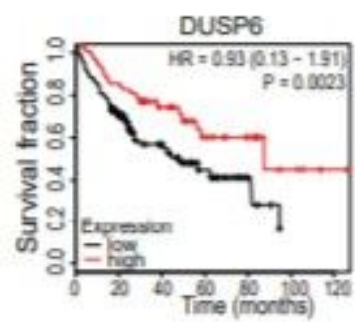

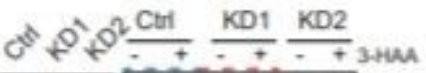

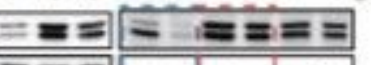

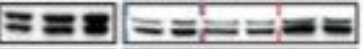

- - - - - - - - -
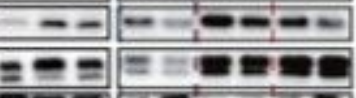

Figure 3

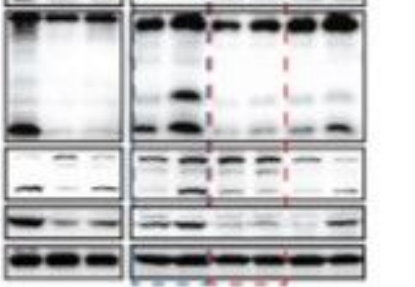

$\mathrm{G}$
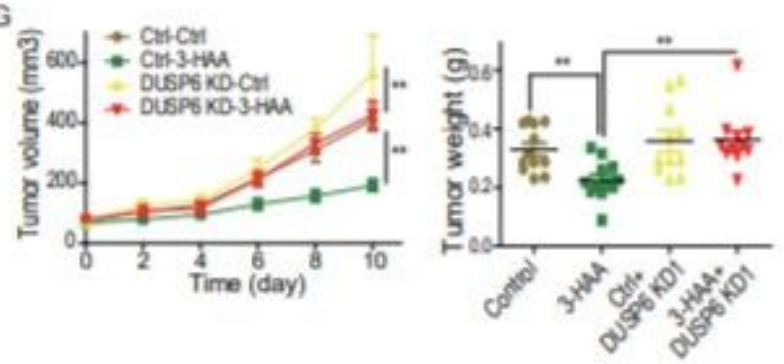
3-HAA induces tumor cell apoptosis by upregulating DUSP6 expression A. The Heatmap of the top consistently upregulated genes in SMMC7721, Hep3B, and SMMC97H. Deep sequencing was used to profile gene expression in HCC SMMC7721, Hep3B, and SMMC97H cells. HCC cells were treated with 100 $\mu \mathrm{M}$ 3-HAA for 1, 8, or $24 \mathrm{~h}$. B. Top consistently upregulated genes were individually verified using quantitative PCR and immunoblotting. C. DUSP6 expression reversely correlated with overall survival of HCC patients, as analyzed by the K-M plotter. The total patient number was 415 . The HCC patients were divided by the median of KMO expression value. D. Effects of DUSP6 knockdown on the growth of HCC cells. Cells were treated with $100 \mu \mathrm{M}$ 3-HAA for the indicated time. DUSP6 was stably knocked down in SMMC7721 cells using lentivirus-generated shRNA. E. Effects of DUSP6 knockdown on HCC cell apoptosis. SMMC7721 cells were treated with $100 \mu \mathrm{M}$ of 3-HAA for $12 \mathrm{~h}$, stained for Annexin V, and analyzed by flow cytometry. F. Effects of DUSP6 knockdown on 3-HAA-activated apoptosis signaling. SMMC7721 cells depleted of DUSP6 were treated with $100 \mu \mathrm{M} 3$-HAA for $24 \mathrm{~h}$. G. DUSP6 knockdown recovered 3-HAA-suppressed xenograft growth. Animals were treated with $3-\mathrm{HAA}$ ( $100 \mathrm{mg} / \mathrm{kg} \cdot \mathrm{day})$ for seven days, then sacrificed on day 10 . SMMC7721 cells were subcutaneously injected into mice. The middle graph shows xenograft weights in different groups. Photographs on the right show representative tumors in different groups. 
A

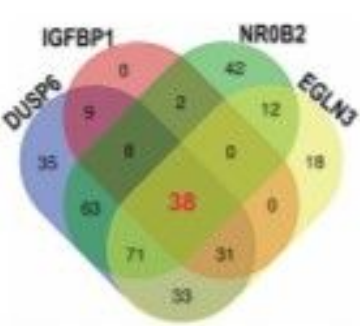

D

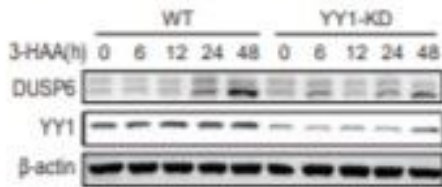

G

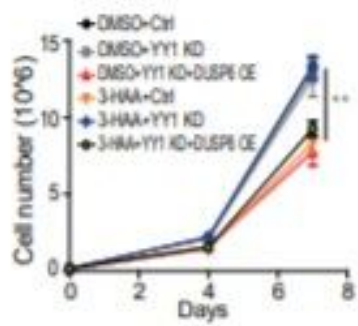

H

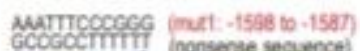

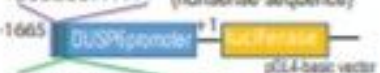

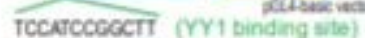

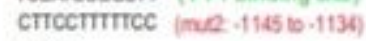
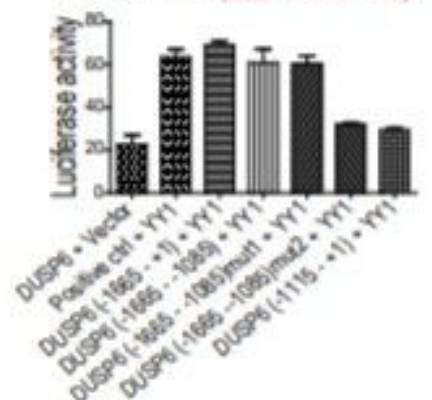

B

3Hareguated TFs on 4 geres

chromalin proteins promoler region

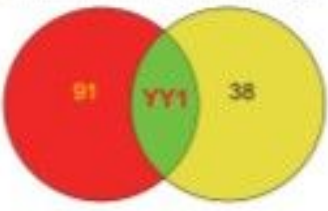

E
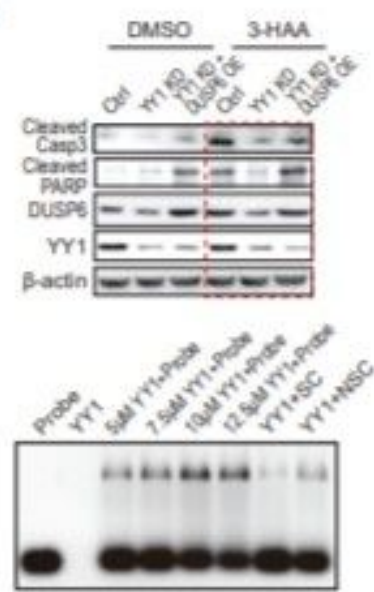

J

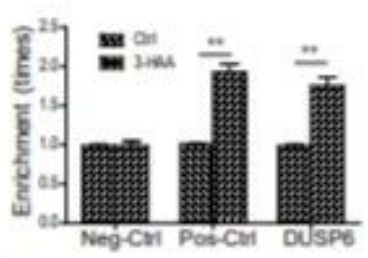

K

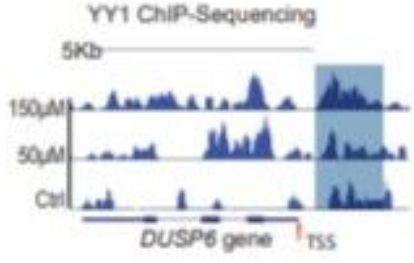

C

Figure 4

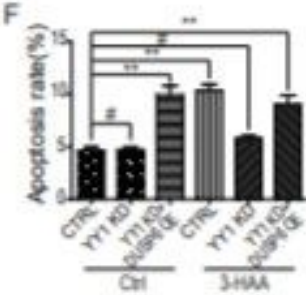

L
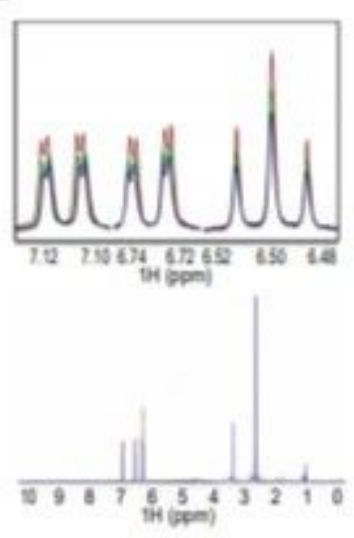

M

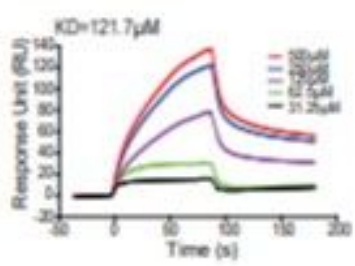

Figure 4

3-HAA upregulated DUSP6 expression by binding with transcription factor YY1 A. The transcription factors potentially binding with promoters of top 4 genes upregulated by 3 -HAA. B. The common proteins between 3-HAA-increased chromatin binding proteins and predicted transcription factors binding to the DUSP6 promoter region. C. 3-HAA increases YY1 nuclear translocation. SMMC7721 cells were treated with $100 \mu \mathrm{M}$ of 3-HAA for 8 hours. D. YY1 knockdown abolished 3-HAA-induced DUSP6 expression. SMMC7721 cells were treated with $100 \mu \mathrm{M}$ of 3-HAA for the indicated time. E. The effect of YY1 knockdown on 3-HAA-activated apoptosis signaling. The concentration of 3-HAA was $100 \mu \mathrm{M}$. F. The effect of YY1 knockdown on 3-HAA-suppressed HCC cell growth. The concentration of 3-HAA was 100 $\mu M$. The **: $P<0.01$. G. The effect of $Y Y 1$ knockdown on 3-HAA-induced HCC apoptosis, analyzed by flow 
cytometry. The concentration of 3-HAA was $100 \mu \mathrm{M}$. The **: P $<0.01$. H. Transcription activity of YY1 on the DUSP6 promoter, as determined in a luciferase reporter assay. The schematic depicts the plasmid encoding luciferase under the control of the DUSP6 promoter, which was truncated or mutated as indicated. I. 3-HAA binding to YY1 determined by electrophoretic mobility shift assay (EMSA). The synthesized oligonucleotide was labeled at the 5 ' terminus with FAM, and $Y Y 1$ was purified using a Hitrap heparin column. J. 3-HAA increased YY1 enrichment on the DUSP6 promoter, analyzed by chromatin immunoprecipitation (ChIP) and quantitative PCR (QPCR). The dose of 3-HAA was $100 \mu \mathrm{M}$. K. The ChIPsequencing analysis of YY1 on the DUSP6 gene. The HCC cells were treated with the indicated dose of 3HAA prior to ChIP-sequencing. L. NMR measurement of direct binding between 3-HAA and YY1. T1r NMR spectra for $6 \mathrm{~b}$ (red) alone or in the presence of SPOP at $2.5 \mathrm{mM}$ (blue), $5 \mathrm{mM}$ (cyan), or $10 \mathrm{mM}$ (green). The STD spectrum for $6 \mathrm{~b}$ was recorded in the presence of $5 \mathrm{mM}$ SPOP. M. 3-HAA bound YY1 protein as shown by surface plasmon resonance. Graphs of equilibrium unit response versus concentrations are shown. The estimated Kd was $121.7 \mu \mathrm{M}$.

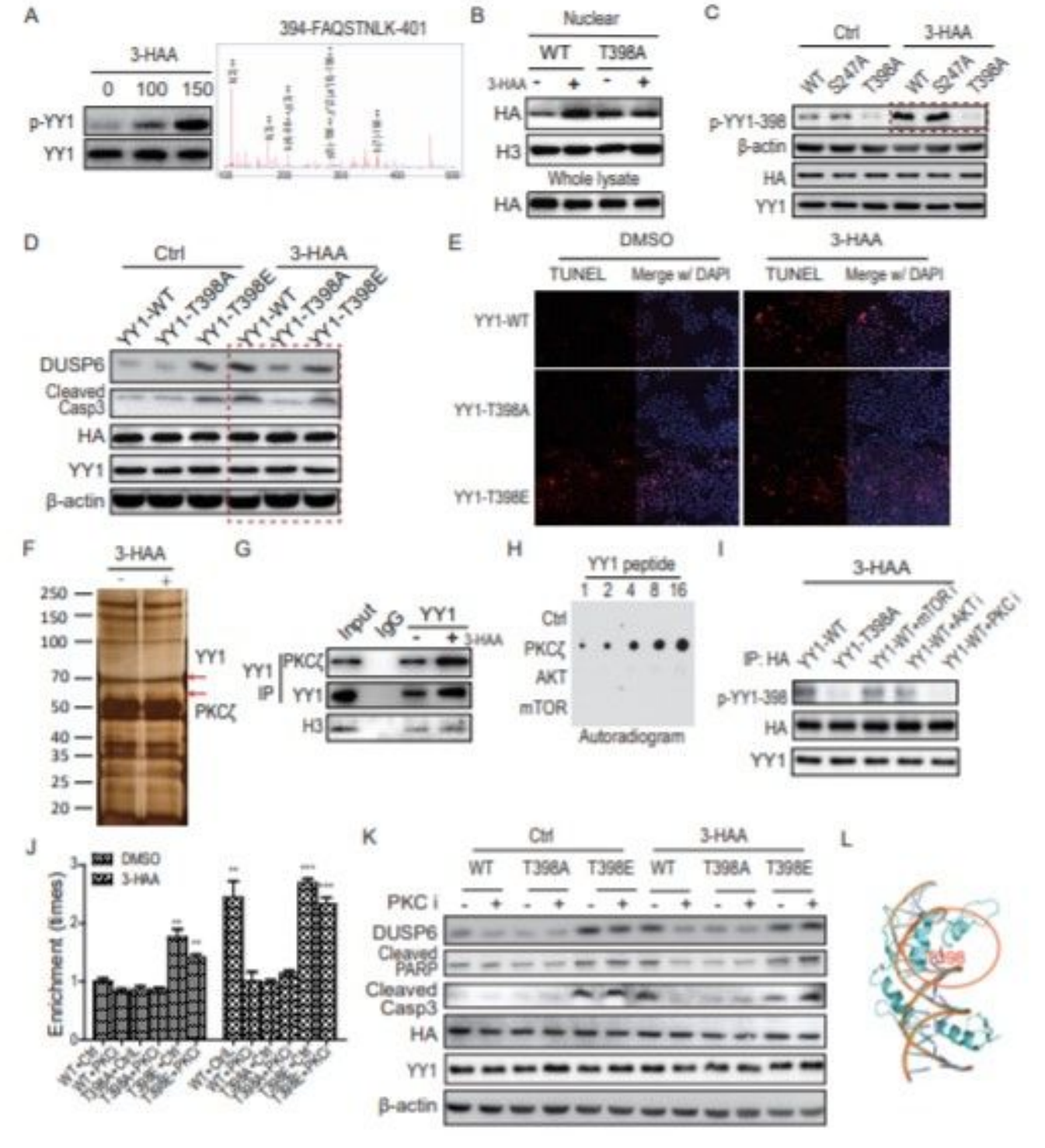




\section{Figure 5}

PKCZ phosphorylates YY1 at Thr 398 in response to 3-HAA A. The YY1 phosphorylation was analyzed by the immunoblotting and the mass-spectrometry. The YY1 was blotted on the enriched phospho-proteins from SMMC7721 cells. The YY1 modification was analyzed by the mass-spectrometry following YY1 immunoprecipitation. B. The YY1 mutation of T398A attenuates the 3-HAA-increased YY1 nuclear accumulation. The concentration of 3-HAA was $100 \mu \mathrm{M}$ and treated for $24 \mathrm{~h}$. C. The T398A but not S247A mutation abolished 3-HAA-induced YY1 phosphorylation. The YY1 was conjugated with HA tag. The YY1 phosphorylation was detected by the T398 phospho-specific antibody. D. The mutation of T398E in YY1 promoted DUSP6 expression, whereas the T398A mutation suppressed DUSP6 upregulation. The YY1 were fused with HA tag. E. The YY1 mutation of T398A reduces the 3-HAA-induced apoptosis, analyzed by the TUNEL assay. F. 3-HAA increased PKC $\zeta$ binding to YY1, analyzed by coimmunoprecipitation and mass-spec. G. 3-HAA increased PKC $\zeta$ binding to YY1, analyzed by immunoblots. H. The kinase screening for T398 phosphorylation of YY1. The kinase candidates were predicted by online tools NetPhos 3.1 (www.cbs.dtu.dk/services/NetPhos) (Blom et al., 2004) and GPS 5.0 (gps.biocuckoo.cn)(Song et al., 2012). I. The effect of kinase inhibitors on 3-HAA-induced YY1 phosphorylation. The YY1 phosphorylation was detected by the T398 phospho-specific antibody. The

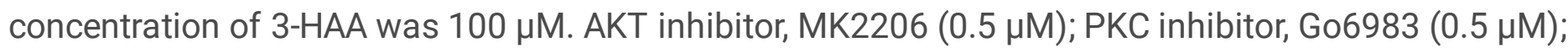
mTOR inhibitor, rapamycin $(0.1 \mu \mathrm{M})$. L. The proposed 3-HAA binding model with YY1. 


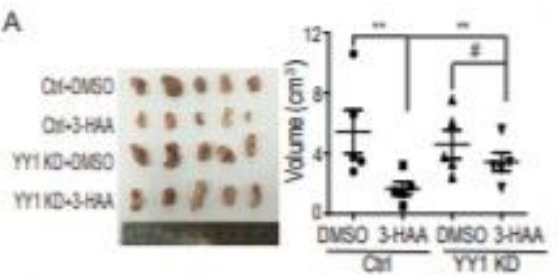

B

D

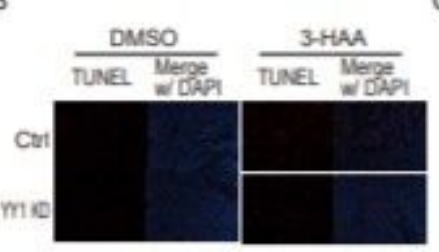

C
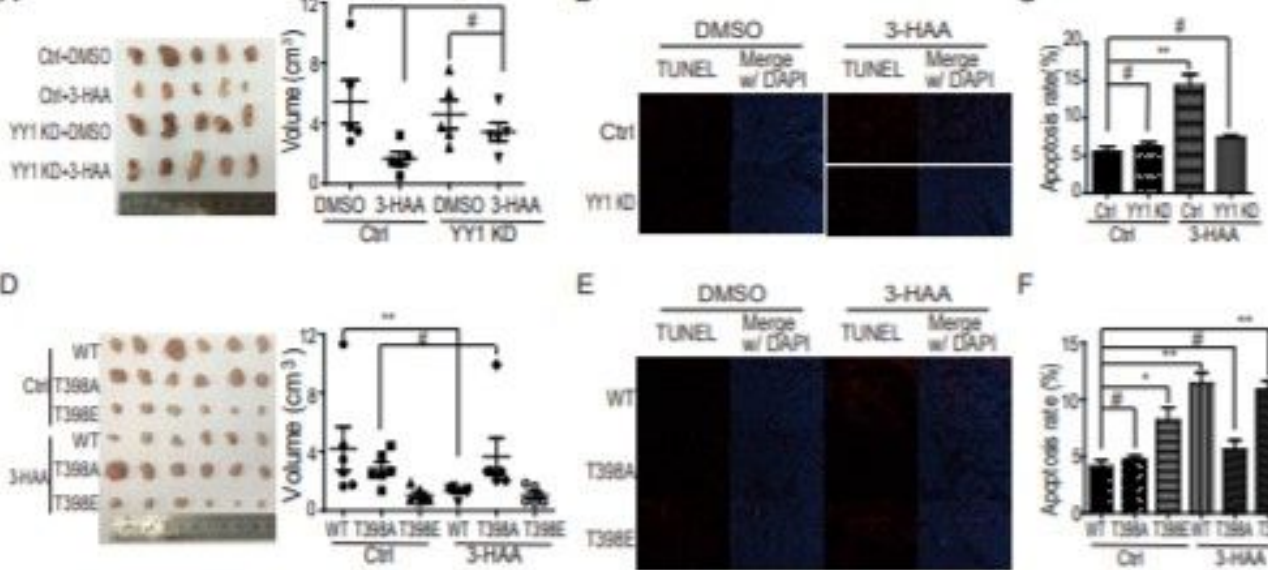

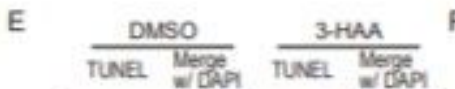
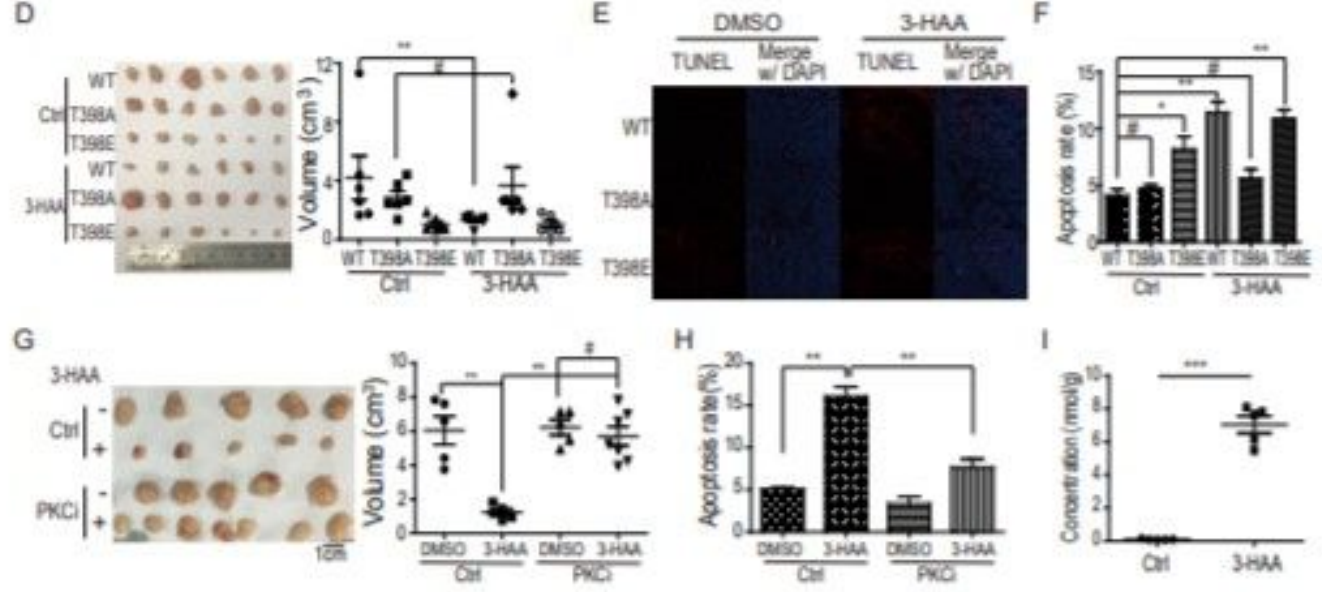

J

K
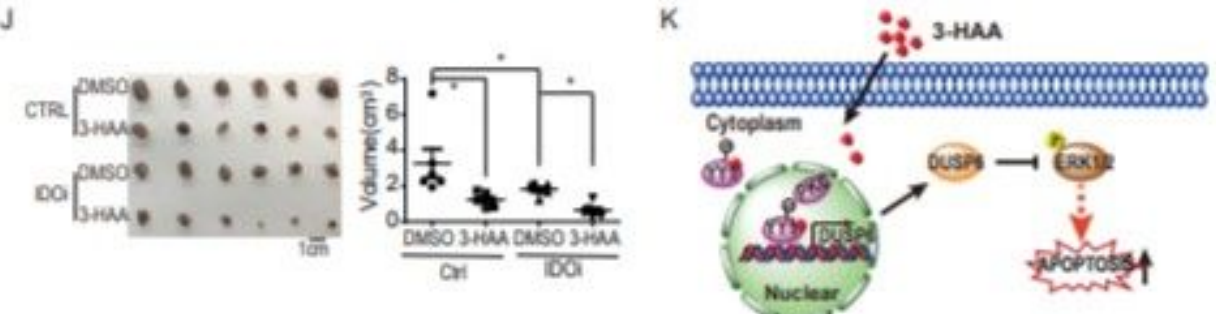

\section{Figure 6}

T398 phosphorylation of YY1 is critical for 3-HAA-suppressed HCC xenografts growth A. YY1 knockdown recovered the 3-HAA-inhibited HCC xenografts growth. B. \& C. The apoptosis analysis in xenografts by TUNEL assay and the flow cytometry. The histogram presents as mean $\pm S D(*: P<0.05 ; * *$ : $P<0.01)$. D. The T398A mutation of YY1 abolished the 3-HAA-inhibited HCC xenografts growth. E. \& F. The apoptosis analysis in xenografts by TUNEL assay and the flow cytometry. The histogram presents as mean \pm SD (*: $P<0.05$; $\star *: P<0.01$.). G. The PKC inhibitor reverted the HCC xenografts growth inhibited by 3-HAA. H. The apoptosis analysis in xenografts by the flow cytometry. The histogram presents as mean $\pm \mathrm{SD}\left({ }^{*}: \mathrm{P}<\right.$ 0.05 ; $* *: P<0.01$.). I. The concentration of 3-HAA in tumors post-administration. The 3-HAA content in xenografts was detected by LC-MS/MS. J. 3-HAA synergizes with IDO inhibitors to HCC xenograft growth. The dose of both 3-HAA and Epacadostat (IDO inhibitor) were $100 \mathrm{mg} / \mathrm{kg}$.day, respectively. K. The working model of 3-HAA-inducing apoptosis. Note: The mouse xenografts were generated by the 
inoculation of $1.5 \times 10^{\wedge} 6$ of SMMC7721 cells into the armpit of rear limb. The 3-HAA ( $100 \mathrm{mg} / \mathrm{kg}$.day) was administered by intraperitoneal injection for 7 days. Tumor volumes are presented as mean \pm SD $(*$ : $P<0.05 ; * \star: P<0.01$.). Photographs on the right show representative xenografts in different groups. 